\title{
Measurement of Muon Neutrino Quasielastic Scattering on a Hydrocarbon Target at $\mathrm{E}_{\nu} \sim 3.5 \mathrm{GeV}$
}

\section{Guillermo Arturo Fiorentini Aguirre}

\author{
Advisor: \\ Hélio da Motta Filho
}

Centro Brasileiro de Pesquisas Físicas

Rio de Janeiro, August 2013 
Dedicado a mi Madre... 


\section{Acknowledgements}

There is a great number of people that I want to thank for their help and support to complete this work. First, I would like to thank my advisor Dr. Hélio da Motta for his guidance and patience. Special thanks to Dr. David Schmitz for sharing his knowledge and never-ending enthusiasm to work; I have learnt a lot from him and this work wouldn't be possible without his invaluable guidance. Also, many thanks to Dr. Jorge Morfin for his constant support during my stay at Fermilab.

Particular gratitude to all present and former MINER $\nu \mathrm{A}$ collaborators; in special the CBPF group lead by Dr. Hélio da Motta: Jose Palomino, David Martinez, Cesar Castromonte, Kenyi Hurtado and Cesar Sotelo. The success of a project as big as MINER $\nu \mathrm{A}$ is only possible thanks to the effort of all the talented people working in it and it has been a pleasure to work with all of them. I also want to thank all fellow students and friends I have met during the years I spent working at CBPF and Fermilab.

Finally, I am most especially and particullary grateful to my parents and family members for their extraordinary encouragement; gratitude to them is beyond what words can describe.

This work has been possible thanks to CAPES, Brazil, for the scholarship received between 2009 and 2013. 


\section{Abstract}

In this thesis, we present the measurement of the charged-current quasi-elastic $\nu_{\mu}$ interaction on polystyrene scintillator $(\mathrm{CH})$ in the MINER $\nu \mathrm{A}$ detector at neutrino energy between 1.5 $\mathrm{GeV}$ and $10.0 \mathrm{GeV}(\sim 3.5 \mathrm{GeV})$. The data used was taken between March and July 2010, with a total of $9.42 \times 10^{19}$ protons-on-target. Interactions were selected by requiring a negative muon and low calorimetric recoil energy separated from the interaction vertex. The analysis was performed on 29,620 charged current quasi-elastic interaction candidates in MINER $\nu$ A's fine-grained scintillator tracker region. The measurement is reported as a flux-averaged single differential cross section in $Q^{2}$ and compared with the prediction of different theoretical models in Monte Carlo simulations. Difference in shape between the measured $d \sigma / d Q_{Q E}^{2}$ and the expectation from a model based on independent nucleons in a relativistic fermi gas is found. 


\section{MINER $\nu \mathrm{A}$ Collaboration}

G. A. Fiorentini ${ }^{1}$, D. W. Schmitz ${ }^{2,3}$, P. A. Rodrigues ${ }^{4}$, L. Aliaga ${ }^{5,6}$, O. Altinok ${ }^{7}$, B. Baldin ${ }^{3}$,

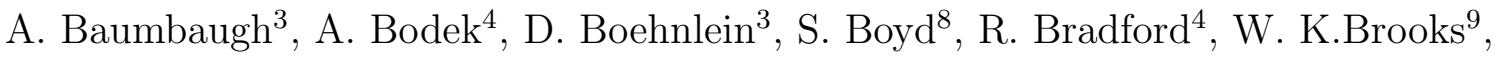
H. Budd ${ }^{4}$, A. Butkevich ${ }^{10}$, D. A. Martinez Caicedo ${ }^{1,3}$, C. M. Castromonte ${ }^{1}$, M. E. Christy ${ }^{11}$, H. Chung ${ }^{4}$, J. Chvojka ${ }^{4}$, M. Clark $^{4}$, H. da Motta ${ }^{1}$, D. S. Damiani ${ }^{5}$, I. Danko ${ }^{8}$, M. Datta ${ }^{11}$, M. Day ${ }^{4}$, R. DeMaat ${ }^{3}$, J. Devan ${ }^{5}$, E. Draeger ${ }^{12}$, S. A. Dytman ${ }^{8}$, G. A. Díaz ${ }^{6}$, B. Eberly ${ }^{8}$, D. A. Edmondson ${ }^{5}$ J. Felix ${ }^{1} 3$, L. Fields ${ }^{1} 4$, T. Fitzpatrick ${ }^{3}$, A. M. Gago ${ }^{6}$, H. Gallagher ${ }^{7}$, C. A. George ${ }^{8}$, J. A. Gielata ${ }^{4}$, C. Gingu ${ }^{3}$, B. Gobbi ${ }^{14}$, R. Gran ${ }^{12}$, N. Grossman ${ }^{3}$, J. Hanson ${ }^{4}$, D. A. Harris ${ }^{3}$, J. Heaton ${ }^{12}$, A. Higuera ${ }^{13}$, I. J. Howley ${ }^{5}$, K. Hurtado ${ }^{1,15}$, M. Jerkins ${ }^{16}$, T. Kafka ${ }^{7}$, J. Kaisen ${ }^{4}$, M. O. Kanter ${ }^{5}$, C. E. Keppel ${ }^{11}$, J. Kilmer ${ }^{3}$, M. Kordosky ${ }^{5}$, A. H. Krajeski ${ }^{5}$, S. A. Kulagin ${ }^{10}$, T. Le ${ }^{17}$, H. Lee ${ }^{4}$, A. G. Leister ${ }^{5}$, G. Locke ${ }^{17}$, G. Maggi ${ }^{9}$, E. Maher ${ }^{18}$, S. Manly ${ }^{4}$, W. A. Mann ${ }^{7}$, C. M. Marshall ${ }^{4}$, K. S. McFarland ${ }^{4,3}$, C. L. McGivern ${ }^{8}$, A. M. McGowan ${ }^{4}$, A. Mislivec ${ }^{4}$, J. G. Morfín ${ }^{3}$, J. Mousseau ${ }^{19}$, D. Naples ${ }^{8}$, J. K. Nelson ${ }^{5}$, G. Niculescu ${ }^{20}$, I. Niculescu ${ }^{20}$, N. Ochoa ${ }^{6}$, C. D. O'Connor ${ }^{5}$, J. Olsen ${ }^{3}$, B. Osmanov ${ }^{19}$, J. Osta ${ }^{3}$, J. L. Palomino ${ }^{1}$, V. Paolone ${ }^{8}$, J. Park ${ }^{4}$, C. E. Patrick ${ }^{14}$, G. N. Perdue ${ }^{4}$, C. Peña ${ }^{9}$, L. Rakotondravohitra ${ }^{3}$, R. D. Ransome ${ }^{17}$, H. Ray ${ }^{19}$, L. Ren $^{8}$, C. Rude ${ }^{12}$, K. E. Sassin ${ }^{5}$, H. Schellman ${ }^{14}$, R. M. Schneider ${ }^{5}$, E. C. Schulte ${ }^{17}$, C. Simon ${ }^{21}$, F. D. Snider ${ }^{3}$, M. C. Snyder ${ }^{5}$, J. T. Sobczyk ${ }^{22,3}$, C. J. Solano Salinas ${ }^{15}$, N. Tagg ${ }^{23}$, W. Tan ${ }^{11}$, B. G. Tice ${ }^{17}$, G. Tzanakos ${ }^{24}$, J. P. Velásquez ${ }^{6}$, J. Walding ${ }^{5}$, T. Walton ${ }^{11}$, J. Wolcott ${ }^{4}$, B. A. Wolthuis ${ }^{5}$, N. Woodward ${ }^{12}$, G. Zavala ${ }^{13}$, H. B. Zeng ${ }^{4}$, D. Zhang ${ }^{5}$, L. Y. Zhu, ${ }^{11}$ B. P.Ziemer ${ }^{21}$

Centro Brasileiro de Pesquisas Físicas, Rio de Janeiro, Brazil ${ }^{1}$

Enrico Fermi Institute, University of Chicago, Chicago, Illinois, USA ${ }^{2}$

Fermi National Accelerator Laboratory, Batavia, Illinois, USA ${ }^{3}$

University of Rochester, Rochester, New York, New York, USA ${ }^{4}$

Department of Physics, College of William \& Mary, Williamsburg, Virginia 23187, USA 5

Sección Física, Departamento de Ciencias, Pontificia Universidad Católica del Perú, Lima, Peru ${ }^{6}$

Physics Department, Tufts University, Medford, Massachusetts, USA ${ }^{7}$

Department of Physics and Astronomy, University of Pittsburgh, Pittsburgh, Pennsylvania, USA ${ }^{8}$

Departamento de Física, Universidad Técnica Federico Santa María, Valparaíso, Chile ${ }^{9}$

Institute for Nuclear Research of the Russian Academy of Sciences, Moscow, Russia ${ }^{10}$

Hampton University, Dept. of Physics, Hampton, VA, USA ${ }^{11}$

Department of Physics, University of Minnesota - Duluth, Duluth, Minnesota, USA ${ }^{12}$

Campus León y Campus Guanajuato, Universidad de Guanajuato,Guanajuato, Mexico. ${ }^{13}$ Northwestern University, Evanston, Illinois ${ }^{14}$

Universidad Nacional de Ingeniería, Lima, Peru ${ }^{15}$

Department of Physics, University of Texas, Austin, Texas, USA ${ }^{16}$

Rutgers, The State University of New Jersey, Piscataway, New Jersey, USA ${ }^{17}$ Massachusetts College of Liberal Arts, MA $01247^{18}$

University of Florida, Department of Physics, Gainesville, FL ${ }^{19}$

James Madison University, Harrisonburg, Virginia, USA 20

Department of Physics and Astronomy, University of California, Irvine, Irvine, California, USA ${ }^{21}$

Institute of Theoretical Physics, Wroclaw University, Wroclaw, Poland ${ }^{22}$

Department of Physics, Otterbein University, Westerville, OH, USA ${ }^{23}$

Department of Physics, University of Athens, Athens, Greece ${ }^{24}$ 


\section{Contents}

$\begin{array}{ll}\text { Acknowledgements } & \text { ii }\end{array}$

$\begin{array}{lll}\text { Abstract } & \text { iii }\end{array}$

The MINER $\nu$ A Collaboration $\quad$ iv

1 Introduction $\quad 1$

2 Neutrino Physics $\quad 2$

2.1 Introduction . . . . . . . . . . . . . . . . . . . 2

2.2 History . . . . . . . . . . . . . . . . . . . . . . 2

2.3 The Standard Model . . . . . . . . . . . . . . . . . . . . . 5

2.3 .1 Weak Interactions . . . . . . . . . . . . . . . . 7

2.3.2 Neutrino Properties . . . . . . . . . . . . . . . . 8

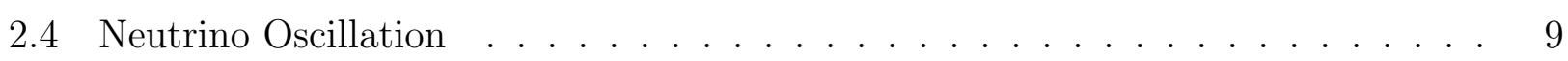

2.5 Neutrino Interactions . . . . . . . . . . . . . . . . . . 12

2.5.1 Quasi-elastic scattering $(\mathrm{QE}) \ldots \ldots \ldots \ldots . \ldots \ldots$

2.5.2 Resonant single pion production (RES) . . . . . . . . . . . . . . 19

2.5.3 Deep inelastic scattering (DIS) f . . . . . . . . . . . . 20

2.5.4 Final state interactions $($ FSI $) \ldots \ldots \ldots \ldots . \ldots \ldots$

3 MINER $\nu$ A Experiment $\quad 23$

3.1 The NuMI beamline . . . . . . . . . . . . . . . . . . . . 23

3.2 The MINER $\nu$ A detector . . . . . . . . . . . . . . . . . . . . 25

3.3 The scintillating strips f . . . . . . . . . . . . . . . . . . . 28

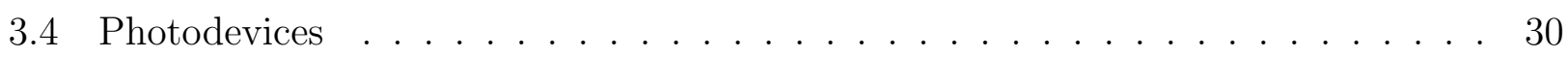

3.5 Calorimeters . . . . . . . . . . . . . . . . . 30

3.5.1 Electromagnetic calorimeter . . . . . . . . . . . . . . 31

3.5 .2 Hadronic calorimeter . . . . . . . . . . . . . . . . . . . . 31

3.6 Nuclear targets . . . . . . . . . . . . . . . . . . . . . . . 32 
3.7 Electronic and Data Acquisition (DAQ) . . . . . . . . . . . . . . 33

3.8 Data Collected by the MINER $\nu$ A Experiment . . . . . . . . . . . . . . . 34

4 Reconstruction and Event Selection 36

4.1 Introduction . . . . . . . . . . . . . . . . . . 36

4.2 Event Sample . . . . . . . . . . . . . . . . . . . . . . . . 36

4.2 .1 Data . . . . . . . . . . . . . . . . . . 36

4.2 .2 Monte Carlo . . . . . . . . . . . . . . . . . . . . 36

4.3 Event Reconstruction . . . . . . . . . . . . . . . . . . . . . . 39

4.3 .1 Muon Reconstruction . . . . . . . . . . . . . . . . . . . . 41

4.3 .2 Recoil Energy reconstruction . . . . . . . . . . . . . . . . . . . . 44

4.4 Event Selection . . . . . . . . . . . . . . . . . . . . . . 45

4.4 .1 Muon Identification . . . . . . . . . . . . . . . . . . . 46

4.4 .2 Fiducial Volume . . . . . . . . . . . . . . . . . . . 47

4.4.3 Reconstructed Neutrino Energy . . . . . . . . . . . . . . . . 47

4.4 .4 Number of Isolated Blobs . . . . . . . . . . . . . . . . . . . . . . 48

4.4 .5 Recoil Energy . . . . . . . . . . . . . . . . . . . . . . . . . 48

4.5 Selected Sample . . . . . . . . . . . . . . . . . . . . . . 53

5 Measuring the Differential Cross section $d \sigma / d Q_{Q E}^{2} \quad 56$

5.1 Introduction . . . . . . . . . . . . . . . . . 56

5.1 Background Constraint and Subtraction . . . . . . . . . . . . 57

5.1 .2 Unfolding Detector Smearing . . . . . . . . . . . . . . . . . . . 59

5.1 .3 Efficiency Correction . . . . . . . . . . . . . . . . . . 60

5.1 .4 Normalization . . . . . . . . . . . . . . . . . . . . . . 61

5.2 Systematics errors . . . . . . . . . . . . . . . . . . . 62

5.2 .1 Neutrino Flux . . . . . . . . . . . . . . . . . . . . 63

5.2 .2 Muon Reconstruction . . . . . . . . . . . . . . . . . . 63

5.2 .3 Recoil Reconstruction . . . . . . . . . . . . . . . . . 63

5.2 .4 Primary Interaction . . . . . . . . . . . . . . . . . . . . . 64

5.2 .5 Hadron Interaction . . . . . . . . . . . . . . . . . . . 65

5.2 .6 Other Sources . . . . . . . . . . . . . . . . . . . . . 65

5.3 Results . . . . . . . . . . . . . . . . . . . 66

5.4 Comparison with Models . . . . . . . . . . . . . . . . . . 68

6 Conclusions $\quad 72$

$\begin{array}{ll}\text { A CCQE Candidates Distributions } & 74\end{array}$ 
C Results

Glossary

Bibliography 


\section{List of Figures}

2.1 The measured and expected electron energy spectrum in beta decay. . . . . . . . 3

2.2 Leptonic part of the interaction Lagrangian $(l=e, \mu$ or $\tau)$. . . . . . . . . . 7

2.3 Two possible neutrino mass hierarchies, where $\Delta m_{\text {atm }}^{2}$ is equivalent to $\Delta m_{23}^{2}$ and

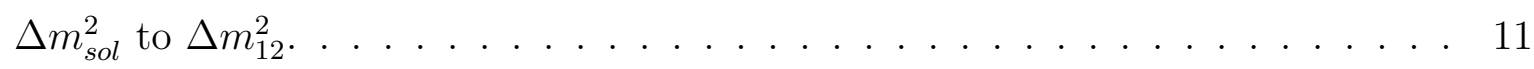

2.4 Total neutrino per nucleon charged current cross sections (for an isoscalar target) divided by neutrino energy as a function of energy. Predictions provided by the NUANCE generator. Figure taken from [42] where the reference to all data points can be found. . . . . . . . . . . . . . . . . . . 13

2.5 Total anti-neutrino per nucleon charged current cross sections divided by neutrino energy as a function of energy. Predictions provided by the NUANCE generator. Figure taken from [42] where a reference to all data points can be found. . . . . . . . . . . . . . . . . . . . 14

2.6 Muon neutrino CCQE cross section per neutron as a function of neutrino energy as measured by MiniBooNE along with LSND and NOMAD [53]. . . . . . . . . 18

3.1 NuMI beamline components. . . . . . . . . . . . . . . . . . 23

3.2 NuMI configurations. Low Energy and Medium Energy, plot generated with FLUKA [64]. . . . . . . . . . . . . . . . . . . . . . . . . . . 24

3.3 Top view of the MINER $\nu$ A detector. . . . . . . . . . . . . . . . 25

3.4 Schematic view of the MINER $\nu \mathrm{A}$ detector. . . . . . . . . . . . . . . 26

3.5 Detector active module, featured, $\mathrm{X}, \mathrm{U}$ and $\mathrm{V}$ planes. Note the $\pm 60^{\circ}$ rotation of the planes $\mathrm{U}$ and $\mathrm{V}$ relative to the X planes. . . . . . . . . . . . . . . . . 27

3.6 Detector ative module. Structure of a module is depicted on the right. . . . . . . 28

3.7 Module of the electromagnetic calorimeter. Structure of modules is depicted on the right. . . . . . . . . . . . . . . . . . . . . 28

3.8 Module of the hadronic calorimeter. Structure of the modules with alternating Fe and scintillating planes is depicted on the right. . . . . . . . . . . . . . . 29

3.9 Transversal cut of the triangular scintillating prism used in the Inner Detector. . 29 
3.10 Scintillating prisms arranged to form a plane. Each prism holds an optical fiber along its full length. . . . . . . . . . . . . . . . . . .

3.11 Position and configuration of each nuclear target in MINER $\nu \mathrm{A}$ nuclear target region. Figure from [65]

3.12 The different periods of data taking in the MINER $\nu \mathrm{A}$ experiment during the low energy configuration of the NuMI beamline.

4.1 Comparison of the invariant production cross section between the FTFP_BERT model in GEANT4 and CERN NA49 hadron production data as a function of the Feynman scaling variable and the transverse momentum of the outgoing particle.

4.2 Correction to the neutrino and anti-neutrino flux in the MINER $\nu \mathrm{A}$ detector calculated as the ratio of the flux after and before applying the weights from the hadron production data comparisons. $\nu_{\mu}$ refers to the muon neutrino flux when NuMI's magents are focusing positive mesons and $\bar{\nu}_{\mu}$ to the muon anti-neutrino flux when focusing negative mesons.

4.3 Corrected flux as a function of neutrino energy used in the analysis. $\nu_{\mu}$ refers to the muon neutrino flux when NuMI's magnets are focusing positive mesons and $\bar{\nu}_{\mu}$ to the muon anti-neutrino flux when focusing negative mesons. . . . . . . .

4.4 The time distribution of hits in the MINER $\nu$ A detector during a NuMI beam spill. The different colored peaks are identified as events that represent a neutrino interaction.

4.5 The MINER $\nu$ A event display. Top: a charged current neutrino event in the tracker region with a reconstructed negative muon exiting from the back (reconstructed track is shown as a green line) in the three views of the MINER $\nu \mathrm{A}$ detector. Bottom: the same event whose muon is exiting MINER $\nu \mathrm{A}$ and entering MINOS. This muon has been reconstructed by both detectors and the resulting tracks merged. . . . . . . . . . . . . . . . . . .

4.6 Left: The tracking efficiency of muons generated by interactions inside the tracker region of the MINER $\nu \mathrm{A}$ detector times the matching to MINOS efficiency in data and MC as a function of the muon momentum measured by MINOS. Right: The comparison between data and $\mathrm{MC}$ of the same efficiency where the horizontal red line represents the average value. Errors in both plots are statistical errors only. . . . . . . . . . . . . . . . . . . .

4.7 The MINER $\nu \mathrm{A}$ event display showing one view (the $\mathrm{X}$ view) of a non-CCQE interaction candidate and the different recoil energy classifications used in its reconstruction 
4.8 The MINER $\nu \mathrm{A}$ event display showing one view (the $\mathrm{X}$ view) of a simulated CCQE interaction where both, the final state muon and proton are visible. . . .

4.9 The MINER $\nu \mathrm{A}$ event display showing one view (the $\mathrm{X}$ view) of a simulated CCQE interaction where the final state proton is below detection threshold and cannot be detected.

4.10 The number of isolated blobs found away from the vertex but in the fiducial volume in data and the $\mathrm{MC}$ simulation. . . . . . . . . . . . . . . .

4.11 Non-vertex recoil energy distributions of CC interactions in the fiducial volume and with less than three isolated blobs. . . . . . . . . . . . . . . . .

4.12 Non-vertex recoil energy distributions of CC interactions in the fiducial volume and with less than three isolated blobs in bins of $Q_{\mathrm{QE}}^{2}$.

4.13 Efficiency, purity and their product as a function of a potential non-vertex recoil energy cut for each bin of $Q_{\mathrm{QE}}^{2}$ considered in the analysis. The vertical red line in each plots is the selected cut that correspond to the minimum cut where the efficiency is $95 \%$ or bigger. . . . . . . . . . . . . . . . . . .

4.14 Non-vertex recoil energy versus the reconstructed $Q_{\mathrm{QE}}^{2}$ distribution of CC interactions in the fiducial volume and with less than three isolated blobs according to the Monte Carlo simulation. CCQE events are in red and non-CCQE events are in blue.

4.15 Distribution of CCQE event candidates as a function of $E_{\nu}^{\mathrm{QE}}$ and $Q_{\mathrm{QE}}^{2}$. The MC sample have been scaled to match the data POT (POT normalization). . . . . . 54

4.16 Selection efficiency as a function of generated $E_{\nu}^{\mathrm{QE}}$ (left) and $Q_{\mathrm{QE}}^{2}$ (right). . . . . 55

4.17 Selected sample purity as a function of generated $E_{\nu}^{\mathrm{QE}}$ (left) and $Q_{\mathrm{QE}}^{2}$ (right). . 55

5.1 Reconstructed $Q_{\mathrm{QE}}^{2}$ distribution of events in the sideband sample. . . . . . . . . 58

5.2 Background scale needed to the background in order to have perfect data-MC agreement in the reconstructed $Q_{\mathrm{QE}}^{2}$ distribution of events in the Sideband sample. 59

5.3 Background subtracted distribution of events in bins of reconstructed $Q_{\mathrm{QE}}^{2}$ as defined in equation 4.6 (left). The ratio between data and MC (right). . . . . . .

5.4 Migration matrix for the $Q_{\mathrm{QE}}^{2}$ bins in the MINER $\nu$ A detector. Right plot's axis shows the actual $Q_{\mathrm{QE}}^{2}$ bins in $\mathrm{GeV}^{2}$. Left plot's axis shows the number of bins. Notice that underflow and overflow bins are considered. . . . . . . . . . . . 60

5.5 Background subtracted and unfolded distribution of events in bins of true $Q_{\mathrm{QE}}^{2}$ (left). The ratio between data and $\mathrm{MC}$ (right). . . . . . . . . . . . . . . .

5.6 Background subtracted, unfolded and efficiency corrected distribution of events in bins of true $Q_{\mathrm{QE}}^{2}$ (left). The ratio between data and MC (right). . . . . . . . 
5.7 Fractional uncertainties on the muon neutrino charged current event rate at the MINER $\nu$ A detector. . . . . . . . . . . . . . . . . . . . . 64

5.8 Differential cross section in bins of reconstructed $Q_{\mathrm{QE}}^{2}$ as defined in equation 4.6 (left). The ratio between data and MC (right). The MC has been normalized to the number of POT in data (POT Normalization) . . . . . . . . . . . . . 66

5.9 Fractional uncertainties for the diferential cross section as function of reconstructed $Q_{\mathrm{QE}}^{2} \ldots \ldots \ldots \ldots \ldots \ldots \ldots$

5.10 Differential cross section in bins of reconstructed $Q_{\mathrm{QE}}^{2}$ as defined in equation 4.6 (left). The ratio between data and MC (right). The MC has been normalized to the total cross section in data (Area Normalization). . . . . . . . . . . . . . . 67

5.11 Fractional uncertainties for the diferential cross section as function of reconstructed $Q_{\mathrm{QE}}^{2}$. Shape uncertainties. . . . . . . . . . . . . 68

5.12 Cross section results and an absolute comparison with GENIE and additional models in NuWro. Top plot is a direct comparison. In the bottom plot all cross sections have been divided by GENIE prediction and presented as a function of $Q_{\mathrm{QE}}^{2}$ in a logarithmic scale to show better low $Q_{\mathrm{QE}}^{2}$ values.

5.13 Cross section results and an shape comparison with GENIE and additional models in NuWro. Top plot is a direct comparison. In the bottom plot all cross sections have been divided by GENIE prediction and presented as a function of $Q_{\mathrm{QE}}^{2}$ in a logarithmic scale to show better low $Q_{\mathrm{QE}}^{2}$ values. . . . . . . . . . 71

A.1 The reconstructed muon energy distribution of CCQE candidates. . . . . . . . . 74

A.2 The reconstructed muon $\theta$ angle distribution of CCQE candidates. . . . . . . . . 75

A.3 The reconstructed muon energy distribution in bins of reconstructed muon angle of CCQE candidates. . . . . . . . . . . . . . . . . . 75

A.4 The reconstructed muon angle distribution in bins of reconstructed muon energy of CCQE candidates. . . . . . . . . . . . . . . . . . . . . 76

A.5 The reconstructed muon $\phi$ angle distribution of CCQE candidates. . . . . . . . 76

A.6 The reconstructed muon angle vs energy distribution of CCQE candidates. . . . 77

B.1 Selection efficiency and sample purity as a function of generated muon energy. . 78

B.2 Selection efficiency and sample purity as a function of generated muon $\theta$ angle. . 79

B.3 Selection efficiency and sample purity as a function of $E_{\nu}^{\mathrm{QE}}$ as defined in equation 4.5 using generated muon momentum and angle. . . . . . . . . . . . . . . . . . 79

B.4 Selection efficiency and sample purity as a function of $Q_{\mathrm{QE}}^{2}$ as defined in equation 4.6 using generated muon momentum and angle. . . . . . . . . . . . . . . . . 80

B.5 Selection efficiency and sample purity as a function of generated muon $\phi$ angle. . 80 


\section{List of Tables}

2.1 Three matter fermion generations. Each generation consists of five different representations of the gauge group. . . . . . . . . . . . . 5 5

2.2 Particles in the Standard Model and their properties as listed in [37]. . . . . . . 6

2.3 Values for $g_{V}$ (vector), $g_{A}$ (axial), $g_{L}$ (left) and $g_{R}$ (right) coupling constants for the known fermion fields. . . . . . . . . . . . . . . . . . 8

2.4 Measured values of the three-flavor neutrino oscillation paremeters [37]. . . . . . 11

2.5 Measured values of the axial mass, $M_{A}$, in heavy-target high-statistics neutrino experiments. A $Q^{2}$ cut was used in some of the results in an attemp to avoid events where the Impulse Aproximation (independent nucleons) is invalid. . . . . 17

3.1 Charged current events expected at each nuclear target. . . . . . . . . . . . . 33

3.2 Some parameters and requirements for the electronics at MINER $\nu \mathrm{A} . \quad$. . . . . . 33

5.1 Primary interaction model parameters in GENIE and their $\pm \sigma$ variation to calculate systematics uncertainties in the analysis. . . . . . . . . . . . . . . . 64

5.2 Hadron interaction (FSI) model parameters in GENIE and their $\pm \sigma$ variation to calculate systematics uncertainties in the analysis. . . . . . . . . . . . . 65

5.3 The $\chi^{2}$ per degree of freedom (DOF) values obtained when the measured differential cross section $d \sigma / d Q_{\mathrm{QE}}^{2}$ is compared with the its different predictions. For the absolute comparisons DOF is eight and seven for the shape comparisons. . .

C.1 The calculated muon neutrino flux per proton on target (POT) for the data included in this analysis. This table correspond to figure $4.3 \ldots$. . . . . . . . . 81

C.2 The measurement of the neutrino differential cross-sections in $Q_{Q E}^{2}$, their total (statistical and systematic) uncertainties, and the correlation matrix for these uncertainties. This table correspond to figure $5.8 \ldots \ldots$. . . . . . . . . . . . 81 
C.3 Fractional systematic uncertainties on $d \sigma / d Q_{Q E}^{2}$ associated with (I) muon reconstruction, (II) recoil reconstruction, (III) neutrino interaction models, (IV) final state interactions, (V) flux and (VI) other sources. The rightmost column shows the total fractional systematic uncertainty due to all sources. This table correspond to figure $5.9 \ldots \ldots \ldots$. . . . . . . . . . . . . . 82

C.4 The measurement of the shape of the neutrino differential cross-sections for $Q_{Q E}^{2}<2.0 \mathrm{GeV}^{2}$, their total (statistical and systematic) uncertainties, and the correlation matrix for these uncertainties. This table correspond to figure 5.11. 


\section{Chapter 1}

\section{Introduction}

Due to the discovery of neutrino oscillations (a discovery that was considered one of the most important developments in particle physics in the last years), neutrino physics has been a very active field both experimentally and theoretically. However, neutrino interactions with matter are not yet well understood in the energy region, between $1 \mathrm{GeV}$ and $20 \mathrm{GeV}$, where three processes overlap: charged-current quasi-elastic scattering, resonant pion production and deep inelastic scattering. Neutrino interaction cross section measurements in this energy region are fundamental to understand the behavior and structure of nucleons and nuclei in weak interactions and will also help to minimize systematic uncertainties in current and future neutrino oscillation experiments.

Neutrino physics has entered the stage of precision measurements and several experiments using intense neutrino beams created in high energy particle accelerators were built to allow measurements of neutrino interaction cross sections in detail. One of these experiments is the Main Injector Neutrino Experiment for $\nu-A(\mathrm{MINER} \nu \mathrm{A})$ : A neutrino scattering experiment that uses the NuMI neutrino beam at Fermilab.

In this thesis, we present the results of our work in the MINER $\nu \mathrm{A}$ experiment which consisted on the first measurement of the single differential cross section, $d \sigma / d Q_{Q E}^{2}$, for muon neutrino charged-current quasi-elastic interactions on a hydrocarbon $(\mathrm{CH})$ target in the MINER $\nu \mathrm{A}$ detector at $\sim 3.5 \mathrm{GeV}$. Chapter 2 briefly introduces basic and general aspects of neutrino physics. Chapter 3 describes the NuMI beamline and the MINER $\nu$ A detector. Chapter 4 describes the reconstructino of neutrino interactions in the MINER $\nu \mathrm{A}$ detector and a selection procedure to obtain a CCQE-rich sample. Chapter 5 presents the calculation to obtaing the measurement of the single differential cross section, $d \sigma / d Q_{Q E}^{2}$, together with a description of the systematic errors considered and a comparison with different theoretical models in Monte Carlo simulations. Finally, chapter 6 summarizes the results obtained. 


\section{Chapter 2}

\section{Neutrino Physics}

\section{$2.1 \quad$ Introduction}

Elementary particles are the building blocks of all matter in the universe and neutrinos are the most abundant of them. Neutrinos are not rare in the universe; therefore, it is important to have a comprehensive knowledge about them. However, despite their abundance, these chargeless and weakly interacting particles are very difficult to detect; their very small interaction probability requires a very intense flux of neutrinos and a very large detectors. Among the most important sources of neutrinos in the earth are the nuclear fusion reactions inside the sun, cosmic rays bombarding Earth's atmosphere, particle accelerators and the decay of natural radioactive elements.

\subsection{History}

In this section, a briefly description of how neutrinos were discovered and their properties measured in the last decades is given.

Neutrinos were first detected, indirectly, in radioactive $\beta$ decays, where a nuclei turns into a different one when one of its neutrons decays into a proton with the emission of an electron and a neutrino,

$$
n \rightarrow p+\mathrm{e}^{-}+\bar{\nu}
$$

Where $n, p, \mathrm{e}^{-}$and $\bar{\nu}$ represent the neutron, proton, electron and antineutrino respectively. In the absence of the antineutrino, a discrete monoenergetic electron espectrum is expected since energy conservation requires the electron and the proton to share the neutron energy. Indeed, if the neutron decays at rest, the energy of the outgoing electron should be: 


$$
E_{\mathrm{e}^{+}}=\frac{m_{n}^{2}-m_{p}^{2}+m_{\mathrm{e}^{-}}^{2}}{2 m_{n}} .
$$

Where $m$ represents the mass of the particles. On the other hand, in the presence of the antineutrino, a continuos electron energy spectrum that correspond to a three particle final state where the particles share the energy is expected.

In 1911, an experiment realized by Adolf von Bayer, Otto Hahn and Lise Meitner [1] suggested that the energy emitted in the $\beta$ decay has a continuous rather than discrete spectrum. However, it was not until 1927 that Ellis and Wooster [2] stablished, without doubts, that the energy spectrum of the $\beta$ decay is, in fact, continuous. At that time, their observations showed that those electrons are not monoenergetic and were in contradiction with the energy conservation law since, apparently, there was energy loss in the process. This situation is showed schematically in figure 2.1 .

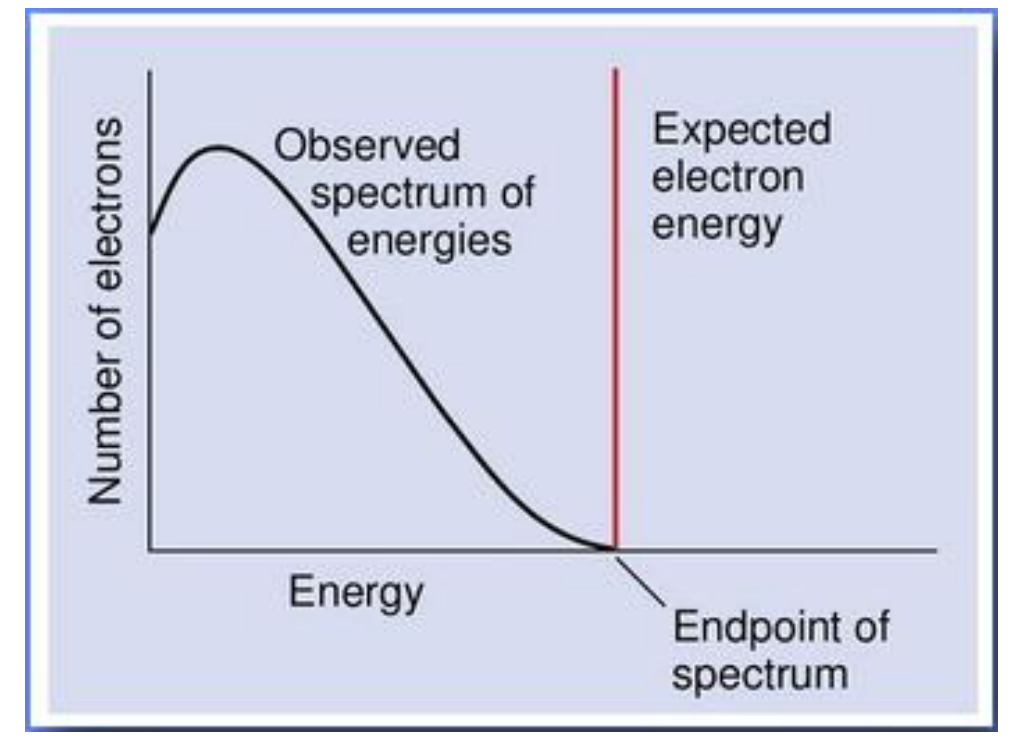

Figure 2.1: The measured and expected electron energy spectrum in beta decay.

In an open letter to Liebe Radioaktive Damen und Herren ${ }^{1}$ in a physics conference in Tubingen, Germany, in 1930, Wolfgang Pauli proposed [3] that the existence of a neutral weakly interacting fermion emitted in the $\beta$ decay could solve the problem. This additional neutral fermion, with mass close to the electron mass and no electric charge, was called neutron by Pauli. When in 1932 Chadwick discovered the neutron that we know today [4] Fermi called Pauli's particle neutrino (little neutron) to diferentiate it from the heavy Chadwick's neutron. In 1933 after comparisons between Fermi [5] and Perrin [6] spectrums, it was postulated that the neutrino should have no mass. And, in 1934, Fermi [7] used Dirac, Heisenberg and Pauli's

\footnotetext{
${ }^{1}$ Dear Radioactive Ladies and Gentlemen.
} 
quantum electrodynamics to formally develop the $\beta$ decay theory. This theory predicts that inverse $\beta$ decay, equation 2.3 , is also possible.

$$
\bar{\nu}+p \rightarrow n+\mathrm{e}^{+} .
$$

In 1956 Reines and Cowan [8] made the first direct observation of the neutrino through inverse $\beta$ decay. They employed a nuclear reactor as a source of anti-neutrinos and a target of 200 liters of water with $40 \%$ of disolved cadmium chloride to observe both, the neutron and the positron, from the reaction. In 1962 an experiment by Leon Lederman, Melvin Schwartz and Jack Steinberger [9] at the Brookhaven Alternating Gradient Synchrotron (AGS) detected a new type of neutrino, the $\nu_{\mu}$. In 1973 the Gargamelle giant bubble chamber at CERN announced the experimental observation of the weak neutral currents [10]. In 1975, a third type of lepton, the $\tau$ lepton, was discovered at the Stanford Linear Accelerator and it was theorized that a third neutrino should also exists. The first detection of actual $\nu_{\tau}$ interactions was made by the DONUT collaboration at Fermilab in 2000 [11], making it the lastest particle in the Standard Model to have been observed.

Experiments with solar neutrinos began in 1968 when Davis [12] revealed a discrepancy between theoretical predictions and the measured solar neutrino flux. This discrepancy came to be known as the solar neutrino problem and led Gribov and Pontecorvo [13], in 1969, to propose the framework of neutrino oscillations [14] [15] to describe the disappearance of solar neutrinos in the traveling distance between the Sun and the earth. This proposal was not accepted by the scientific community at the beginning because there were concerns about the validity of the theoretical solar model at that time and the possible mistakes in Davis' experiment due to its technical difficulty. Then, in 1998, a discrepancy between the expected and the measured flux was also observed in the Super-Kamiokande experiment [16] that registered the apparent disappearance of atmospheric muon neutrinos in a few hundred kilometers of propagation which suggested that neutrinos are really oscillating. However, the first direct observation of neutrino oscillation was made by the Sudbury Neutrino Observatory (SNO) [17], in 2002, thanks to its sensitivity to all three neutrino flavors, confirming Davis' measurement after $~ 30$ years and solving the solar neutrino problem.

In 2003, the KamLAND experiment in Japan observed evidence that electron anti-neutrinos from nuclear reactors also oscillate and in 2006, the K2K and MINOS experiments did the same with muon neutrinos from high energy accelerators. Several additional neutrino experiments have been built in order to verify the neutrino oscillation hypothesis and to study in detail neutrino interactions. These experiments are using neutrinos from nuclear reactors and high energy accelerators to measure neutrino oscillation parameters and cross sections improving accuracy over time. 
It has been a long journey since the pioneers hitherto during which we have witnessed an intense experimental and theoretical activity aimed at a better understanding of neutrino interactions with nucleons and nuclei. The discovery of the neutrinos and neutrino oscillations started a new era of physics. We have found evidences that neutrinos oscillate and, therefore, have mass; a fact that is not predicted by the Standard Model.

\subsection{The Standard Model}

The Standard Model is a coherent and conceptually simple theory that describes all known fundamental particles and how they interact. It is able to succesfully describe three of the four interactions of Nature: the strong, the weak and the electromagnetic interactions, with the latter two unified in an electroweak theory. It is a local gauge theory based on the symmetry group $S U(3)_{C} \times S U(2)_{L} \times U(1)_{Y}$, where $S U(3)_{C}$ describes the strong interaction; and $S U(2)_{L} \times$ $U(1)_{Y}$, the electroweak interaction. The Standard Model is incomplete since it does not describe the fourth interaction, gravity; neutrino masses; and the origin of dark matter and dark energy in the universe. It is, however, the most succesful theory with many measurements confirming its predictions.

According to the Standard Model, all phenomena around us can be explained using a set of fundamental particles: Six quarks, six leptons, force carrier particles called bosons and their corresponding antiparticles. Quarks and leptons are related in pairs, or generations, that differ only by their mass: The lightest and most stable ones make up the first generation, whereas the heavier and less stable particles belong to the second and third generations. Each generation consists of five different representations of the gauge group; two left-handed weak isospin doublets and three right-handed singlets, see table 2.1.

\begin{tabular}{|cc|ccc|}
\hline$L_{L}(1,2,-1 / 2)$ & $Q_{L}(3,2,1)$ & $E_{R}(1,1,-1)$ & $U_{R}(3,1,2 / 3)$ & $D_{R}(3,1,-1 / 3)$ \\
\hline$\left(\begin{array}{c}\nu_{e} \\
e\end{array}\right)_{L}$ & $\left(\begin{array}{c}u \\
d\end{array}\right)_{L}$ & $e_{R}$ & $u_{R}$ & $d_{R}$ \\
$\left(\begin{array}{c}\nu_{\mu} \\
\mu\end{array}\right)_{L}$ & $\left(\begin{array}{c}c \\
s\end{array}\right)_{L}$ & $\mu_{R}$ & $c_{R}$ & $s_{R}$ \\
$\left(\begin{array}{c}\nu_{\tau} \\
\tau\end{array}\right)_{L}$ & $\left(\begin{array}{l}t \\
b\end{array}\right)_{L}$ & $\tau_{R}$ & $t_{R}$ & $b_{R}$ \\
\hline
\end{tabular}

Table 2.1: Three matter fermion generations. Each generation consists of five different representations of the gauge group.

Force carrier particles that mediate the interactions are the eight gluons for the strong interaction; the $W^{ \pm}$and $Z^{0}$ for the weak interaction; the photon for the electromagnetic interaction and also the Higgs boson that explain why some particles have mass. See table 2.2 for a list of all these particles and some of their measured properties. 


\begin{tabular}{|c|c|c|}
\hline \multicolumn{3}{|c|}{ QUARKS } \\
\hline Quarks & Mass & Electric charge \\
\hline $\operatorname{up}(u)$ & $2.3_{-0.5}^{+0.7} \mathrm{MeV} / \mathrm{c}^{2}$ & $+\frac{2}{3}$ \\
\hline down $(d)$ & $4.8_{-0.3}^{+0.7} \mathrm{MeV} / \mathrm{c}^{2}$ & $-\frac{1}{3}$ \\
\hline strange $(s)$ & $95 \pm 5 \mathrm{MeV} / \mathrm{c}^{2}$ & $-\frac{1}{3}$ \\
\hline $\operatorname{charm}(c)$ & $1.275 \pm 0.025 \mathrm{GeV} / \mathrm{c}^{2}$ & $+\frac{2}{3}$ \\
\hline bottom $(b)$ & $4.65 \pm 0.03 \mathrm{GeV} / \mathrm{c}^{2}$ & $-\frac{1}{3}$ \\
\hline top $(t)$ & $173.5 \pm 0.6 \pm 0.8 \mathrm{GeV} / \mathrm{c}^{2}$ & $+\frac{2}{3}$ \\
\hline \multicolumn{3}{|c|}{ LEPTONS } \\
\hline Leptons & Mass & Electric charge \\
\hline electron $(e)$ & $0.510998928 \pm 0.000000011 \mathrm{MeV} / \mathrm{c}^{2}$ & -1 \\
\hline electron neutrino $\left(\nu_{e}\right)$ & $<2 \mathrm{eV} / \mathrm{c}^{2}$ & 0 \\
\hline muon $(\mu)$ & $105.6583715 \pm 0.0000035 \mathrm{MeV} / \mathrm{c}^{2}$ & -1 \\
\hline muon neutrino $\left(\nu_{\mu}\right)$ & $<0.19 \mathrm{MeV} / \mathrm{c}^{2}$ & 0 \\
\hline $\operatorname{tau}(\tau)$ & $1776.82 \pm 0.16 \mathrm{MeV} / \mathrm{c}^{2}$ & -1 \\
\hline tau neutrino $\left(\nu_{\tau}\right)$ & $<18.2 \mathrm{MeV} / \mathrm{c}^{2}$ & 0 \\
\hline \multicolumn{3}{|c|}{ BOSONS } \\
\hline Bosons & Mass & Electric charge \\
\hline photon $(\gamma)$ & $<1 \times 10^{-18} \mathrm{eV} / \mathrm{c}^{2}$ & 0 \\
\hline $\mathrm{W}^{ \pm}$ & $80.385 \pm 0.015 \mathrm{GeV} / \mathrm{c}^{2}$ & \pm 1 \\
\hline $\mathrm{Z}^{0}$ & $91.1876 \pm 0.0021 \mathrm{GeV} / \mathrm{c}^{2}$ & 0 \\
\hline gluon $(\mathrm{g})$ & 0 & 0 \\
\hline Higgs & $\sim 125 \mathrm{GeV} / \mathrm{c}^{2}$ [38] [39] & 0 \\
\hline
\end{tabular}

Table 2.2: Particles in the Standard Model and their properties as listed in [37]. 


\subsubsection{Weak Interactions}

The Standard Model of weak and electromagnetic interactions was first proposed in 1967 by A. Salam [40] and S. Weinberg [41]. The electroweak interaction is based on a local $S U(2) \times U(1)$ gauge symmetry. After spontaneous symmetry breaking via the Higgs mechanism [35], we get for the interaction part of the Lagrangian,

$$
\mathcal{L}_{\text {int }}=\mathcal{L}_{C C}+\mathcal{L}_{N C}+\mathcal{L}_{E M}
$$

The leptonic part of this Langragian is schematically shown in Figure 2.2.

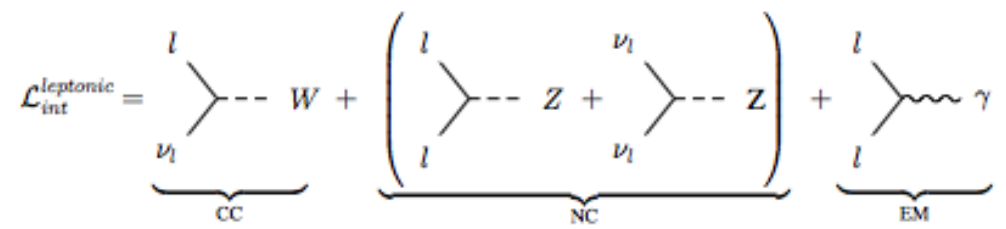

Figure 2.2: Leptonic part of the interaction Lagrangian $(l=e, \mu$ or $\tau)$.

$\mathcal{L}_{C C}$ is the charged current Lagrangian that couples neutrinos and anti-neutrinos via a $W^{ \pm}$boson to their charged lepton partners and vice versa. The second type of interaction, $\mathcal{L}_{N C}$ is the neutral current Lagrangian that couples neutrinos and anti-neutrinos via the $Z^{0}$ boson. Both of them are described by the equations 2.5 and 2.6 respectively; where $W_{\mu}$ and $Z_{\mu}$ represent the heavy gauge boson field, $g$ is the weak coupling constant, $\theta_{W}$ is the Weinberg angle. The last type of interactions, $\mathcal{L}_{E M}$, is the electromagnetic currents that couples charged leptons to the photon field.

$$
\begin{gathered}
-\mathcal{L}_{C C}=\frac{g}{2 \sqrt{2}}\left(j_{W}^{\mu} W_{\mu}+j_{W}^{\mu, \dagger} W_{\mu}^{\dagger}\right) \\
-\mathcal{L}_{N C}=\frac{g}{2 \cos \theta_{W}} j_{Z}^{\mu} Z_{\mu}
\end{gathered}
$$

The leptonic charged weak current $j_{W}^{\mu}$ is given by the form,

$$
j_{W}^{\mu}=2 \sum_{\alpha=e, \mu, \tau} \bar{\nu}_{L, \alpha} \gamma^{\mu} l_{\alpha L}
$$

The leptonic neutral-current term, $j_{Z}^{\mu}$, describes the exchange of the neutral boson $Z^{0}$,

$$
j_{Z}^{\mu}=2 \sum_{\alpha=e, \mu, \tau} g_{L}^{\nu} \bar{\nu}_{\alpha L} \gamma^{\mu} \nu_{\alpha L}+g_{L}^{f} \bar{l}_{\alpha L} \gamma^{\mu} l_{\alpha L}+g_{R}^{f} \bar{l}_{\alpha L} \gamma^{\mu} l_{\alpha R}
$$

Here $\nu_{\alpha L(R)}$ and $l_{\alpha L(R)}$ correspond to the left (right) neutral and charged leptonic fields, while $g_{L}^{\nu}, g_{L}^{f}$, and $g_{R}^{f}$ represent the fermion left and right-handed couplings. Table 2.3 details these values. 


\begin{tabular}{|c|c|c|c|c|}
\hline Fermion & $g_{L}^{f}$ & $g_{R}^{f}$ & $g_{V}^{f}$ & $g_{A}^{f}$ \\
\hline$\nu_{e}, \nu_{\mu}, \nu_{\tau}$ & $+\frac{1}{2}$ & 0 & $+\frac{1}{2}$ & $+\frac{1}{2}$ \\
$e, \mu, \tau$ & $-\frac{1}{2}+\sin ^{2} \theta_{W}$ & $+\sin ^{2} \theta_{W}$ & $-\frac{1}{2}+2 \sin ^{2} \theta_{W}$ & $-\frac{1}{2}$ \\
$u, c, t$ & $\frac{1}{2}-\frac{2}{3} \sin ^{2} \theta_{W}$ & $-\frac{2}{3} \sin ^{2} \theta_{W}$ & $\frac{1}{2}-\frac{4}{3} \sin ^{2} \theta_{W}$ & $+\frac{1}{2}$ \\
$d, s, b$ & $-\frac{1}{2}+\frac{1}{3} \sin ^{2} \theta_{W}$ & $+\frac{1}{3} \sin ^{2} \theta_{W}$ & $-\frac{1}{2}+\frac{2}{3} \sin ^{2} \theta_{W}$ & $-\frac{1}{2}$ \\
\hline
\end{tabular}

Table 2.3: Values for $g_{V}$ (vector), $g_{A}$ (axial), $g_{L}$ (left) and $g_{R}$ (right) coupling constants for the known fermion fields.

The neutrino interactions within the Standard Model are given by equations 2.5 and 2.6, and it is possible to articulate all neutrino interactions [36] within this simple framework. Historically, the neutrino charged-current and neutral-current interactions have been used to study the nature of the weak force in great detail.

\subsubsection{Neutrino Properties}

\section{Flavor}

The Standard Model of particle physics contains three neutrino flavors: $\nu_{e}, \nu_{\mu}$ and $\nu_{\tau}$. Each neutrino forms a doublet with a corresponding charged lepton. The number of neutrinos participating in the electroweak interaction can be determined by the $Z^{0}$ decay width. It was beautifully confirmed at LEP (CERN) [21, 22, 23, 24], long before the observation of the $\nu_{\tau}$, that there are only three light neutrinos. This measurement of the decay width of the $Z^{0}$ boson into neutrinos makes the existence of three, and only three, light (that is, $m_{\nu}<m_{Z} / 2$ ) active neutrinos an experimental fact. When expressed in units of the Standard Model prediction for a single neutrino generation, one gets:

$$
\begin{aligned}
& N_{\nu}=2.994 \pm 0.012 \quad \text { (Standard Model fits to LEP data) } \\
& N_{\nu}=3.00 \pm 0.06 \quad \text { (Direct measurement of invisible } \mathrm{Z} \text { width) }
\end{aligned}
$$

Where $N_{\nu}$ is the number of neutrino flavors. Evidence of the existence of additional sterile neutrinos that does not undergo weak interactions nor interact in any other way (except gravity) by some neutrino oscillation experiments is still an open question and will be discussed later at the end of 2.4 .

\section{Helicity}

Wu showed in the late 1950s that parity is violated in weak interactions [25] and Goldhaber [26] observed that neutrinos have spin antiparallel to their momentum (left-handed) and anti- 
neutrinos have it parallel (right-handed). Therefore, only left-handed neutrinos and righthanded anti-neutrinos are included in the Standard Model.

\section{Mass}

Currently, the absolute values of the neutrino masses are unknown. The Standard Model assumes that neutrinos are massless. However, no fundamental aspect of the Standard Model forbids massive neutrinos and it is quite straightforward to insert neutrino mass terms into the Standard Model Lagrangian. There are two basic methods to generate neutrino mass terms that are both gauge and Lorentz invariant [27].

Dirac mass. This is obtained by introducing extremely heavy right-handed neutrinos, which have not yet been observed. These neutrinos appear in many Grand Unified Theories. The mass term in the Lagrangian is therefore:

$$
\mathcal{L}_{\text {Dirac }}=-\left(\bar{\nu}_{L} M \nu_{R}+\bar{\nu}_{R} M \nu_{L}\right)
$$

where $\nu_{L, R}$ are the neutrino flavour eigenstates and $\mathrm{M}$ is the $3 \times 3$ neutrino mass matrix.

Majorana mass. A massive Majorana neutrino can be created by modifying the Higgs sector in the Standard Model. An additional singlet, doublet or triplet is added to the original Higgs doublet, although this introduces a new mass scale in the form of the Higgs vacuum expectation value. The mass term in the Lagrangian is:

$$
\mathcal{L}_{\text {Majorana }}=\frac{1}{2} \bar{\nu}_{L}^{c} M \nu_{L}+\text { h.c. }
$$

In this case neutrinos are their own anti-particles since $\nu_{L}^{c}$ is a right-handed neutrino. These mass terms violate lepton number conservation by two units and their presence could be indicated by the observation of neutrino double $\beta$ decay, nuclear transitions of the type,

$$
(Z, A) \rightarrow(Z-2, A)+2 e-,
$$

which are only possible in the presence of massive Majorana neutrinos. The non-observation of this transition in current experiments sets a limit to the mass of the electron neutrino of $m_{\nu_{e}}<0.5 \mathrm{eV}$ if $\nu_{e}$ is assumed to be a Majorana particle.

\subsection{Neutrino Oscillation}

The discovery of non-zero neutrino masses is closely related to the discovery of neutrino oscillations. Neutrino oscillations are only possible with massive neutrinos due to a distinction between flavor and mass eigenstates. The principle is analogous to the time evolution of a classical coupled oscillator starting with an excitation that is not a normal mode. For simplicity we 
consider a system with only two neutrinos. Neutrinos produced in charged current interactions are flavor eigenstates denoted as $\nu_{e}$ and $\nu_{\mu}$. Those eigenstates have no well defined masses and are linear superpositions of the mass eigenstates $\nu_{1}$ and $\nu_{2}$ with masses $m_{1}$ and $m_{2}$, respectively:

$$
\begin{gathered}
\left|\nu_{e}\right\rangle=\left|\nu_{1}\right\rangle \cos \theta+\left|\nu_{2}\right\rangle \sin \theta, \\
\left|\nu_{\mu}\right\rangle=-\left|\nu_{1}\right\rangle \sin \theta+\left|\nu_{2}\right\rangle \cos \theta,
\end{gathered}
$$

where $\theta$ is the neutrino mixing angle. At time $t=0$ we have a pure weak eigenstate, say $|\nu(0)\rangle=\left|\nu_{\mu}\right\rangle$. But $\nu_{\mu}$ is a superposition of the mass eigenstates each of which is propagating with the time dependence dictated by the free Hamiltonian. Therefore at a time $t$ the state, using natural units $(\hbar=c=1)$ will be given by

$$
|\nu(t)\rangle=-\left|\nu_{1}\right\rangle \sin \theta e^{-i E_{1} t}+\left|\nu_{2}\right\rangle \cos \theta e^{-i E_{2} t},
$$

where $E_{1,2}=\sqrt{\left(p^{2}+m_{1,2}^{2}\right)} \sim p+\frac{m_{1,2}^{2}}{2 p}$. The probability of finding a neutrino with electron flavor is then

$$
\begin{aligned}
P\left(\nu_{\mu} \rightarrow \nu_{e} ; t\right) & =\left|\left\langle\nu_{e} \mid \nu(t)\right\rangle\right|^{2} \\
& =\sin ^{2} \theta \cos ^{2} \theta\left|-e^{-i E_{1} t}+e^{-i E_{2} t}\right|^{2} \\
& =\sin ^{2} \theta \cos ^{2}\left(\frac{\Delta m^{2} t}{4 E}\right) \\
& =\sin ^{2} \theta \cos ^{2}\left(\frac{\Delta m^{2} L}{4 E}\right)
\end{aligned}
$$

where $\Delta m^{2}=m_{2}^{2}-m_{1}^{2}$ is the squared mass difference and $E=p$. The last line is valid for highly relativistic particles with $\mathrm{L}$ being the travelled distance.

The two-flavor-oscillation scheme can be easily extended to three flavor mixing. The neutrino mixing Pontecorvo-Maki-Nakagawa-Sakata matrix then contains three angles $\theta_{12}, \theta_{13}, \theta_{23}$, one Dirac CP violating phase and possibly two Majorana phases. Furthermore we have three squared mass differences: $\Delta m_{12}^{2}, \Delta m_{13}^{2}$ and $\Delta m_{23}^{2}$. After the recent measurement of non-zero $\theta_{13}$ in accelerator [28] [29] and reactor neutrino experiments [30] [31] [32], all of these parameters, with exception of the $\mathrm{CP}$ violating phase, have been measured and the current best known values are shown in table 2.4 .

The three flavor mixing framework has been well stablished and it succesfully explains solar, atmospheric, reactor and accelerator neutrino oscillation data [37]. Note that only the mass difference squared appears, hence measuring oscillation probabilities will not give absolute values of the neutrino masses. If $\nu_{1}$ is defined as the mass state having the largest component of 


\begin{tabular}{|c|c|}
\hline Parameter & Value \\
\hline $\sin ^{2} \theta_{12}$ & $0.857 \pm 0.024$ \\
$\sin ^{2} \theta_{23}$ & $>0.95$ \\
$\sin ^{2} \theta_{13}$ & $0.098 \pm 0.013$ \\
$\Delta m_{12}^{2}$ & $7.50 \pm 0.20 \times 10^{-5} \mathrm{eV}^{2}$ \\
$\Delta m_{12}^{2}$ & $2.32_{-0.08}^{+0.12} \times 10^{-3} \mathrm{eV}^{2}$ \\
\hline
\end{tabular}

Table 2.4: Measured values of the three-flavor neutrino oscillation paremeters [37].

the electron flavor, the value of $\Delta m_{12}^{2}$ is determined to be positive using the oscillation pattern from solar neutrinos. However, the sign of $\Delta m_{23}^{2} \approx \Delta m_{13}^{2}$ remains unknown. This means that there are two possibles orderings: the "normal hierarchy", where $\nu_{3}$ is the heaviest, and the "inverted hierarchy", in which $\nu_{3}$ is the lightest as shown in figure 2.3.

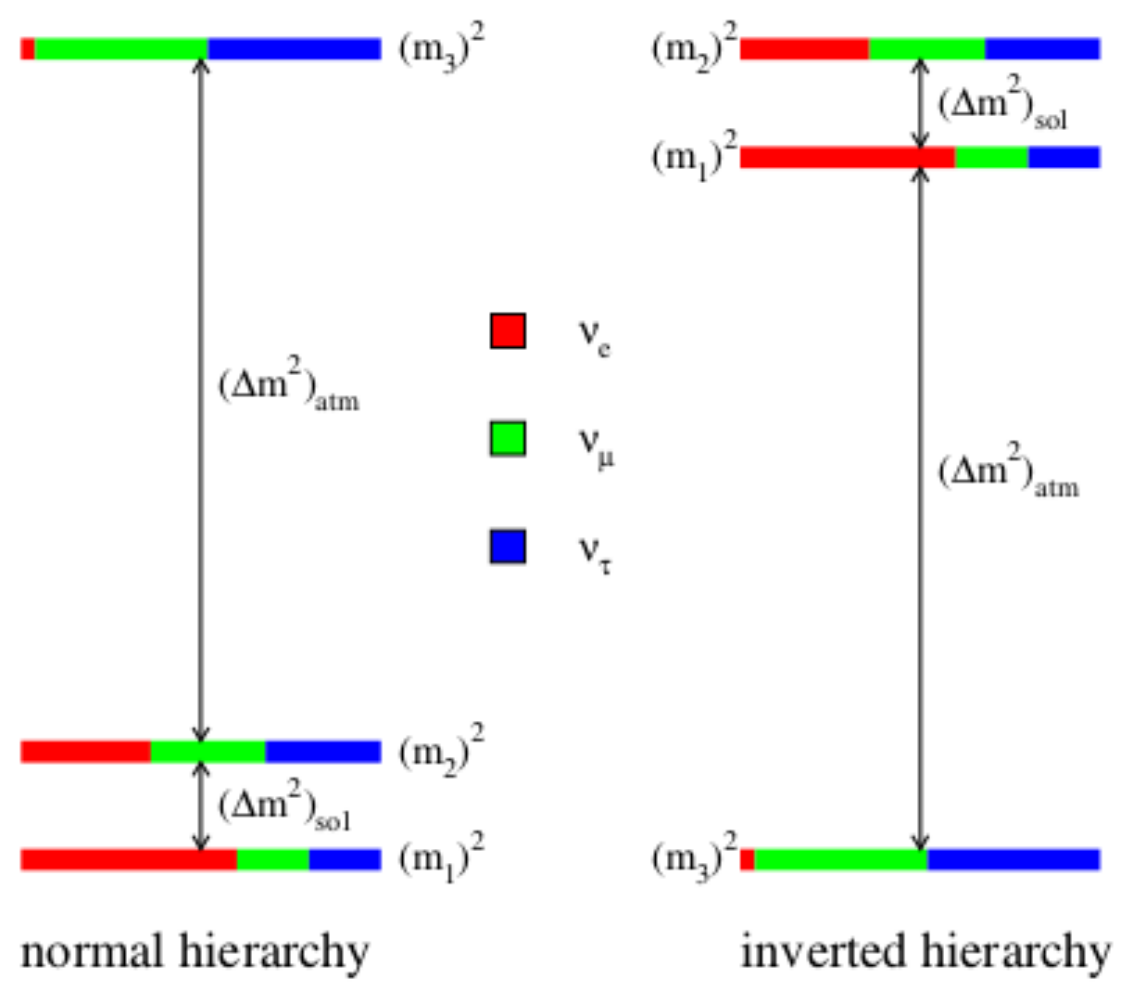

Figure 2.3: Two possible neutrino mass hierarchies, where $\Delta m_{\text {atm }}^{2}$ is equivalent to $\Delta m_{23}^{2}$ and $\Delta m_{\text {sol }}^{2}$ to $\Delta m_{12}^{2}$.

In 1995 the LSND experiment claimed that three neutrinos were not enough to explain their observed oscillations and introduced a sterile neutrino [33]. MiniBooNE results from 2010 showed evidence of muon neutrino to electron neutrino oscillations in the LSND region, consistent with the simple 2-neutrino oscillation interpretation of the LSND results [34]. Both 
results are not conclusive and more advanced analyses of their data and new experiments are currently being considered.

\subsection{Neutrino Interactions}

Since Pauli's prediction of the neutrino, this particle has played an important role in the understanding of the weak interactions. In the last decades, scientists have detected neutrinos from a variety of sources, both natural and man-made. Knowledge of the neutrino interaction cross sections is important and necessary in any precise measurement of neutrino properties. With the advent of new precision oscillation experiments, the demands on our understanding of neutrino interactions is increasing.

In the Standard Model, neutrinos are considered massless and purely left-handed. This is in contrast to the experimental evidence for non-zero mass neutrino as discussed previously. However, even though the non-zero mass is necessary for neutrino oscillations, it is not at all important for neutrino nucleon interactions simply because it is too small. This tiny mass will not affect any of the calculations, therefore, we can assume it to be zero in all that follows.

To understand neutrino interaction cross sections, it is helpful to define different neutrino energy regimes where the same theoretical approximations are valid [42]:

- Thresholdless processes, $E_{\nu}=0-1 \mathrm{MeV}$.

- Low-energy nuclear processes, $E_{\nu}=1-100 \mathrm{MeV}$.

- Intermediate energy cross sections $E_{\nu}=0.1-20 \mathrm{GeV}$.

- High-energy cross sections, $E_{\nu}=20-500 \mathrm{GeV}$.

- Ultra-high-energy neutrinos $E_{\nu}>500 \mathrm{GeV}$.

In this thesis, we will concentrate on the intermediate energy region ${ }^{2}$ where several processes such as quasielastic scattering, neutral current elastic scattering, resonant single pion production, coherent pion production, multipion production, kaon production and deep inelastic scattering, have a contribution. For the total charged-current muon neutrino and anti-neutrino total cross section, however, we can only consider the contributions of the three processes that play the most significant role, $\sigma_{T O T}=\sigma_{Q E}+\sigma_{R E S}+\sigma_{D I S}$ where,

- $\sigma_{Q E}$ : quasi-elastic cross section, $\nu n \rightarrow \mu^{-} p$.

\footnotetext{
${ }^{2}$ This energy region is often called the "transition region" because it corresponds to the boundary between quasielastic scattering (where the neutrino target is a nucleon) on one end and deep inelastic scattering (where the neutrino target is a parton in the nucleon) on the other.
} 
- $\sigma_{R E S}$ : resonant production cross section, $\nu N \rightarrow \mu N^{*}$.

- $\sigma_{D I S}$ : deep inelastic scattering cross section, $\nu N \rightarrow \mu X$.

Historically, adequate theoretical descriptions of quasielastic, resonance-mediated, and deep inelastic scattering have been formulated; however, there is no uniform description which globally describes the transition between these processes or how they should be combined. Moreover, the full extent to which nuclear effects impact this region is a topic that has only recently been appreciated. Figures 2.4 and 2.5 show the prediction of the total charged-current (anti)neutrino cross section and these three components using a Monte-Carlo simulation (NUANCE [43]) together with several experimental results accumulated over many decades using a variety of neutrino target and detector technologies.

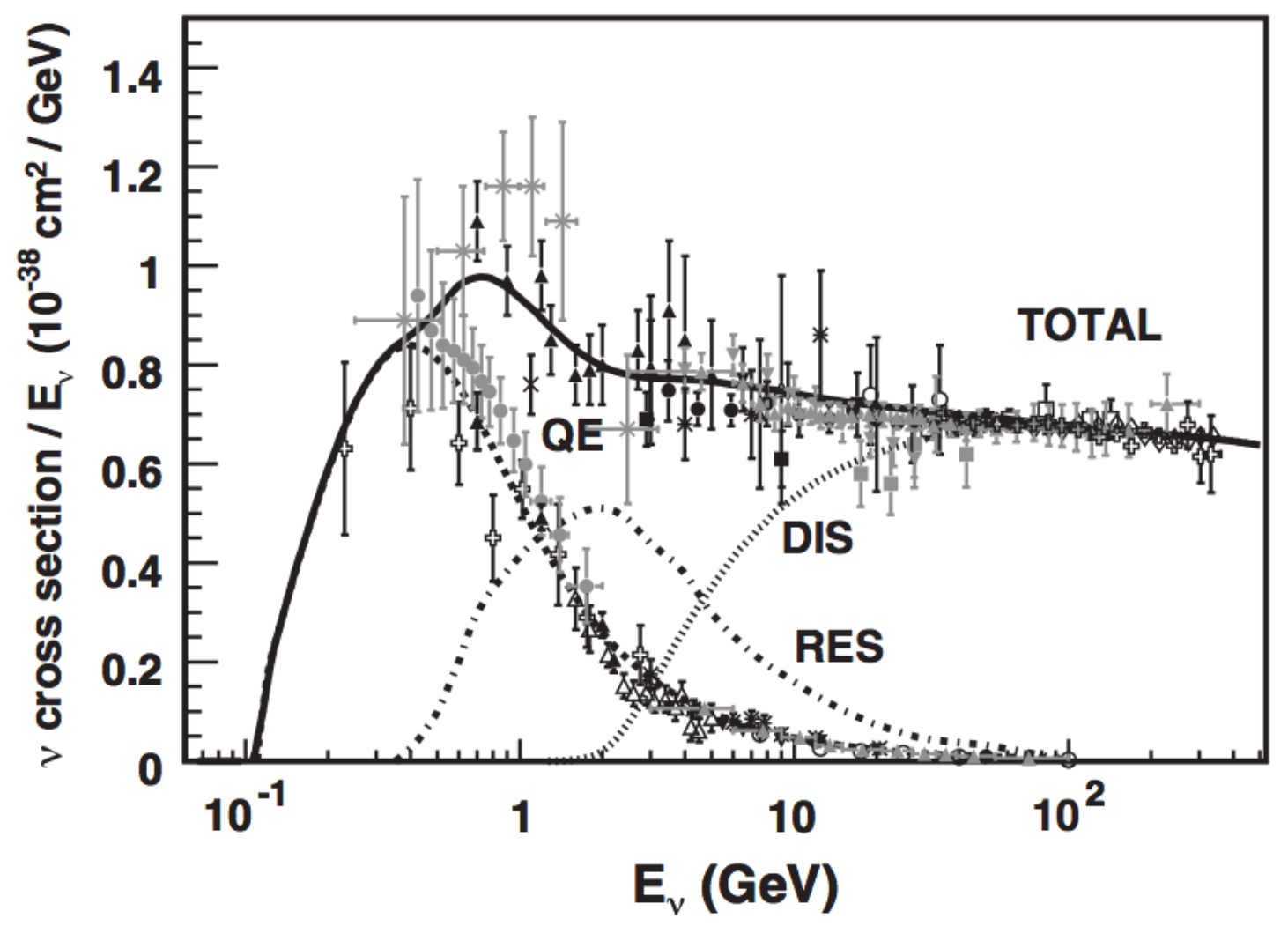

Figure 2.4: Total neutrino per nucleon charged current cross sections (for an isoscalar target) divided by neutrino energy as a function of energy. Predictions provided by the NUANCE generator. Figure taken from [42] where the reference to all data points can be found.

Most of our knowledge of neutrino cross sections in this intermediate energy range comes from early experiments that collected relatively small data samples (few thousand events). But in order to better understand these neutrino cross sections, several experiments K2K, 


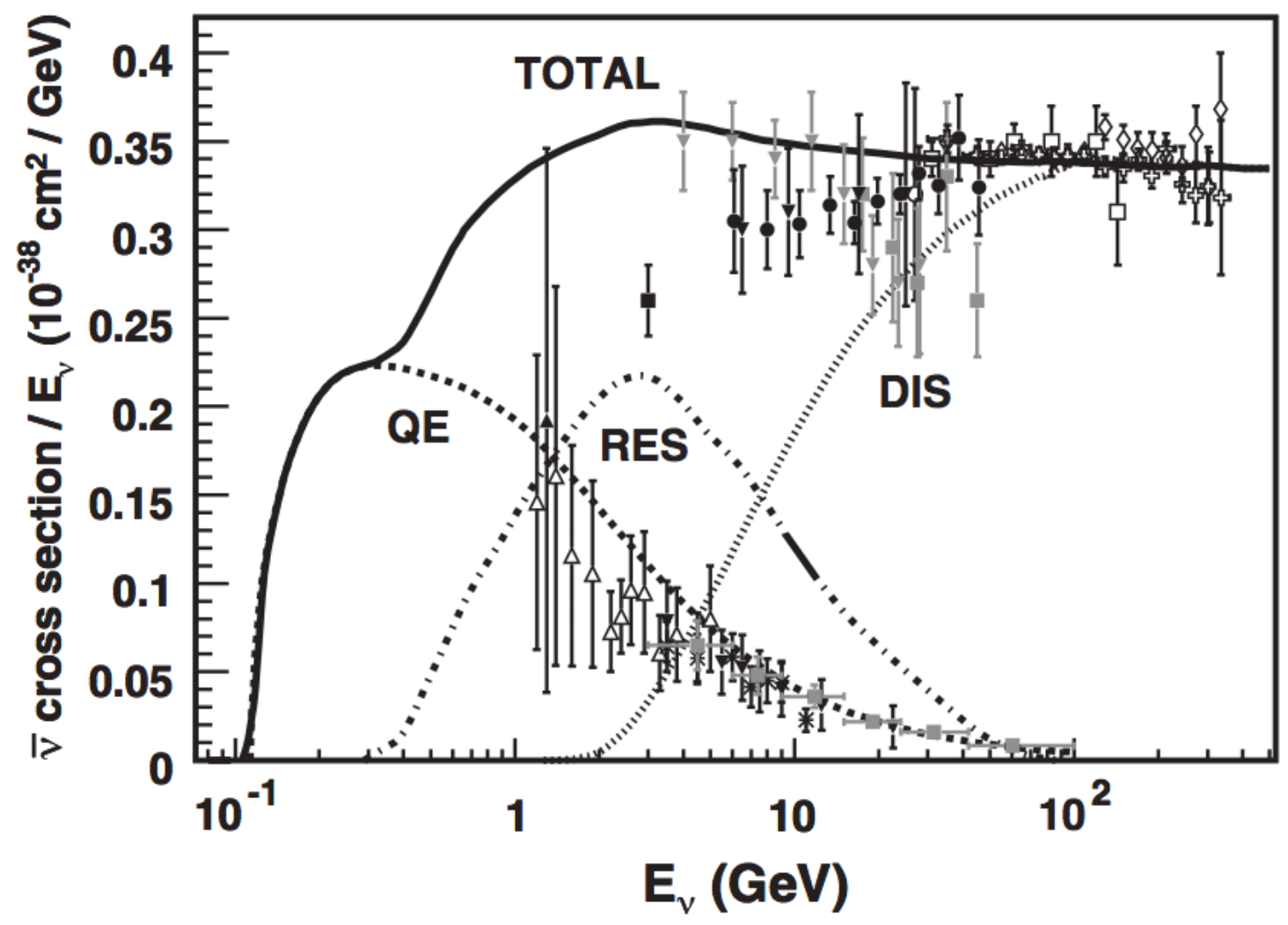

Figure 2.5: Total anti-neutrino per nucleon charged current cross sections divided by neutrino energy as a function of energy. Predictions provided by the NUANCE generator. Figure taken from [42] where a reference to all data points can be found.

MiniBooNE, MINER $\nu$ A, MINOS, NOMAD, and T2K are studying or have studied this intermediate energy region in great detail. New theoretical approaches have also recently emerged. A description of intermediate energy region processes most relevent for the analysis presented in this thesis follows.

\subsubsection{Quasi-elastic scattering (QE)}

Neutrinos can elastically scatter off an entire nucleon liberating a nucleon (or multiple nucleons) from the target. In the case of charged current neutrino scattering, this process is called "quasielastic scattering" and for neutral-current scattering this is traditionally referred to as "elastic scattering". As can be seen in figures 2.4 and 2.5, quasi-elastic scattering is the dominant process in neutrino and anti-neutrino interactions in the $\mathrm{E}_{\nu}<2 \mathrm{GeV}$ region. This energy region is also used by accelerator-based neutrino oscillation experiments. Precise measurement of this process cross section and its dependence to the energy is of fundamental importance for neutrino oscillation experiments. In a charged current neutrino QE interaction, the target neutron is converted to a proton, 


$$
\nu n \rightarrow \mu^{-} p
$$

In the case of an antineutrino interaction, the target proton is converted to a neutron,

$$
\bar{\nu} p \rightarrow \mu^{+} n
$$

The most used theoretical description of this proccess was given in a review on neutrino interactions made by Llewellyn Smith [44] in 1972. Here, the author uses the standard theory of weak interactions considering the neutrino scattering off free nucleons. Nucleons, in this approach, are not point particles so their form factor are considered. All experiments rely heavily on this formalism and according to it, the quasielastic differential cross section can be expressed as

$$
\frac{d \sigma}{d Q^{2}}=\frac{G_{f}^{2} M^{2} \cos ^{2} \theta_{C}}{8 \pi E_{\nu}^{2}}\left[A\left(Q^{2}\right) \pm \frac{s-u}{M^{2}} B\left(Q^{2}\right)+\frac{(s-u)^{2}}{M^{4}} C\left(Q^{2}\right)\right]
$$

where $(-)+$ refers to (anti)neutrino scattering, $G_{F}$ is the Fermi coupling constant, $\theta_{C}$ is the Cabbibo angle, $Q^{2}$ is the squared four-momentum transfer $\left(Q^{2}=-q^{2}>0\right)$ from the leptonic to hadronic system, $M$ is the nucleon mass, $m$ is the lepton mass, $E_{\nu}$ is the incident neutrino energy, and $(s-u)=4 M E_{\nu}-Q^{2}-m^{2}$ is a simple conbination of two Mandelstam invariants. The factors $A\left(Q^{2}\right), B\left(Q^{2}\right)$, and $C\left(Q^{2}\right)$ are functions of the $Q^{2}$-dependent vector, $F_{1}$ and $F_{2}$, axial-vector $F_{A}$, and pseudoscalar $F_{P}$ form factors of the nucleon (the form factors describes how different the nucleon is from a point like particle in the scattering). The explicit dependence is showed in formulas 2.20, 2.21 and 2.22 where the definition $\tau=Q^{2} / 4 M^{2}$ is used.

$$
\begin{gathered}
A\left(Q^{2}\right)=\frac{m^{2}+Q^{2}}{M^{2}}\left[(1+\tau) F_{A}^{2}-(1-\tau) F_{1}^{2}+\tau(1-\tau) F_{2}^{2}\right. \\
\left.+4 \tau F_{1}^{2} F_{2}^{2}-\frac{m^{2}}{4 M^{2}}\left(\left(F_{1}^{2}+F_{2}^{2}\right)^{2}+\left(F_{A}^{2}+2 F_{P}^{2}\right)^{2}-4(1+\tau) F_{P}^{2}\right)\right] \\
B\left(Q^{2}\right)=\frac{Q^{2}}{M^{2}} F_{A}\left(F_{1}+F_{2}\right) \\
C\left(Q^{2}\right)=\frac{1}{4}\left(F_{A}+F_{1}+F_{2}\right)
\end{gathered}
$$

The vector part of the neutrino cross section, $F_{1}$ and $F_{2}$, can also be expresed in terms of the vector electric and magnetic form factors, $G_{E}$ and $G_{M}$. Under the conserved vector current (CVC) hypothesis, these vector electric and magnetic form factors are related to the elastic nucleon form factors in electron scattering $G_{E}^{n}, G_{E}^{p}, G_{M}^{n}$ and $G_{M}^{p}$, 


$$
\begin{gathered}
G_{E}=G_{E}^{p}-G_{E}^{n} \\
G_{M}=G_{M}^{p}-G_{M}^{n} .
\end{gathered}
$$

The vector form factors have been measured in electron scattering experiments and their data was used to parametrize their functional form which is close to a dipole form. One of this parametrizations, referred to as BBBA05 [45], finds a departure of the dipole form of few- $\%$ and is used in current neutrino interaction Monte Carlo simulations. Small contributions to the total cross section from the pseudo-scalar form factor $F_{P}$ is expected for muon neutrino scattering [46]. The only remaining unknown in the model is the nucleon axial form factor.It is custumary to assume for the $Q^{2}$ dependace of the axial form factor a dipole form, equation 2.25 .

$$
F_{A}\left(Q^{2}\right)=\frac{g_{A}}{\left(1+Q^{2} / M_{A}^{2}\right)^{2}} .
$$

The axial form factor depends on two empirical parameters: the value of the axial-vector form factor at $Q^{2}=0, g_{A}=F_{A}(0)=1.2694 \pm 0.0028$ determined from neutron beta decay experiments, and the value of the axial mass $\left(M_{A}\right)$ that can be only measured by neutrino experiments.

To complete the description of charged current QE interactions, a model for nucleons in a nucleus is needed. There has been several approaches over time and most of them use the Impulse Aproximation (IA), based on the assumption that, at large enough 3-momentum tranfer, the nucleus is seen as a collection of independent nucleons. It is also the most common approximation in Monte Carlo simulations used by most experiments. Some of these nuclear models are:

\section{Relativistic Fermi Gas Model (RFG)}

This is the simplest nuclear model, widely used in Monte Carlo simulations. In this model, nucleons form a Fermi gas with an average Fermi momentum and binding energy that were adjusted to reproduce data of electron scattering experiments [47]. For instance, a binding energy of $34 \mathrm{MeV}$ and Fermi momentum of $220 \mathrm{MeV}$ for Carbon is used in the GENIE [48] neutrino Monte Carlo generator.

The main interest in experiments between 1970-1990 was testing the vector-axial vector (VA) nature of the weak interaction and in measuring the axial-vector form factor of the nucleon, topics that were considered particularly important in providing an anchor for the study of NC interactions. Values of $M_{A}$ ranging from $0.65 \mathrm{GeV}$ to $1.09 \mathrm{GeV}$ were obtained during this early period where bubble chamber and spark chamber detectors with deuterium targets (for which 
nuclear effects are minimal) were used. By the end of this period, the neutrino QE cross section could be accurately and consistently described assuming a dipole axial-vector form factor with $M_{A}=1.077 \pm 0.039 \mathrm{GeV}$ and a RFG for the nucleus [49].

Approaches beyond the Fermi gas model have been developed in recent years to incorporate more sophisticated treatments,

\section{$\mathrm{RFG}+$ effective $M_{A}=1.35 \mathrm{GeV} / c^{2}$}

More recent neutrino oscillation experiments use heavy nuclear targets such as carbon, oxygen or iron to improve data rates. They have consistenly measured bigger values of $M_{A}$ than the ones obtained in the old experiments that used hydrogen or deuterium targets, with the only exception of the NOMAD experiment, as shown in table 2.5.

\begin{tabular}{|c|c|c|c|}
\hline Experiment & Target & Cut & $M_{A}$ \\
\hline K2K & Oxygen & $Q^{2}>0.2 \mathrm{GeV}^{2}$ & $1.20 \pm 0.12[50]$ \\
K2K & Carbon & $Q^{2}>0.2 \mathrm{GeV}^{2}$ & $1.14 \pm 0.12[51]$ \\
MINOS & Iron & $Q^{2}>0 \mathrm{GeV}^{2}$ & $1.19 \pm 0.17[52]$ \\
MiniBooNE & Carbon & $Q^{2}>0 \mathrm{GeV}^{2}$ & $1.35 \pm 0.17[53]$ \\
MiniBooNE & Carbon & $Q^{2}>0.25 \mathrm{GeV}^{2}$ & $1.27 \pm 0.17[53]$ \\
NOMAD & Carbon & $Q^{2}>0 \mathrm{GeV}^{2}$ & $1.07 \pm 0.07[54]$ \\
\hline
\end{tabular}

Table 2.5: Measured values of the axial mass, $M_{A}$, in heavy-target high-statistics neutrino experiments. A $Q^{2}$ cut was used in some of the results in an attemp to avoid events where the Impulse Aproximation (independent nucleons) is invalid.

The NOMAD experiment measurement was performed at neutrino energies between 6 and $50 \mathrm{GeV}$ and it is consistent with the old experiments average of $M_{A}$. However, the MINOS experiment, in a similar range of energy, obtained a bigger value. The $\mathrm{K} 2 \mathrm{~K}$ and MiniBooNE experiments, at lower neutrino energies, obtained also bigger values. In addition, a comparison between the total cross section as a function of energy measured by MiniBooNE and NOMAD was performed in [53]. By doing this, an inconsistency between Miniboone, at energies between 0.5 and $1.5 \mathrm{GeV}$, and NOMAD, at energies between 6 and $50 \mathrm{GeV}$ was found and is shown in Figure 2.6. All these inconsistencies are intriguing and led us to think that the underlying model, independent of neutrino energy and nuclear target, to extract $M_{A}$ is too simple.

The difference between old and more recent experiments has been attributed to nuclear effects that have not been taken into account in the simulations. This is one of the reasons why better models of the nucleus are needed to account for nuclear effects. Regardless, an effective value of $M_{A}=1.35 \mathrm{GeV}$ was proposed to explain a higher than the $\mathrm{RFG}$ and $M_{A}=1 \mathrm{GeV}$ 


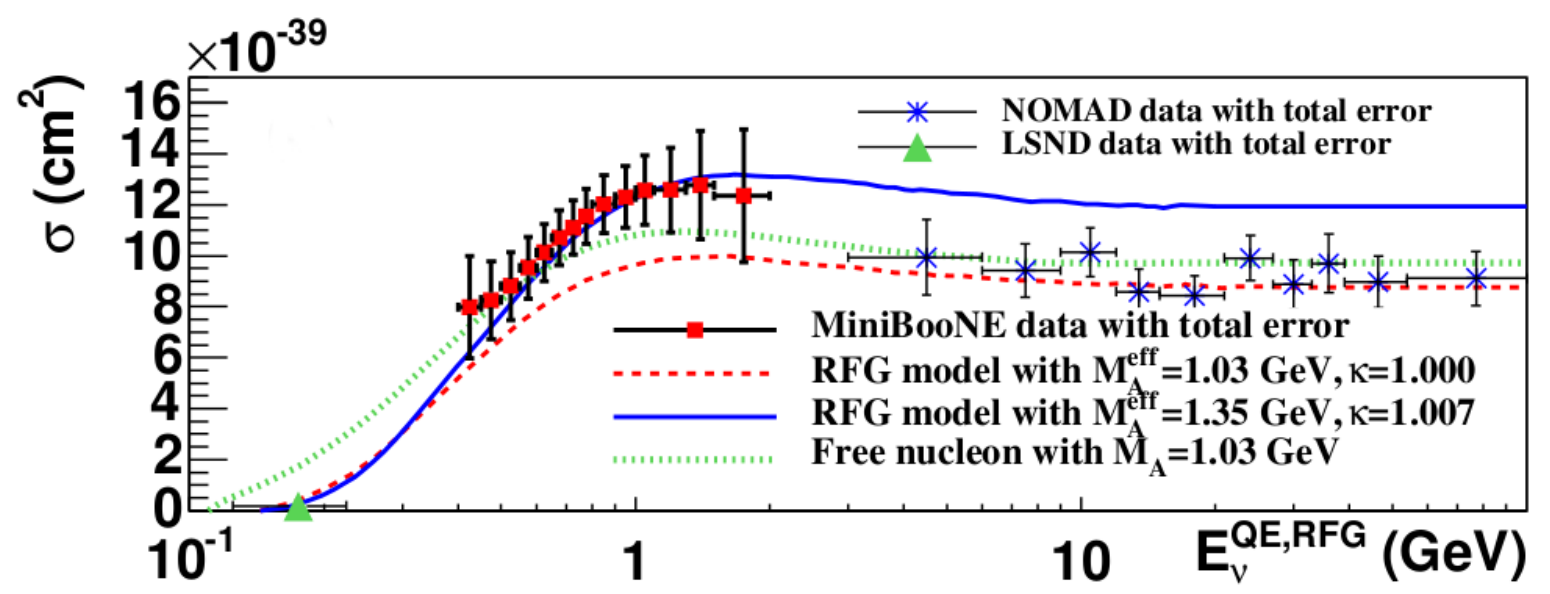

Figure 2.6: Muon neutrino CCQE cross section per neutron as a function of neutrino energy as measured by MiniBooNE along with LSND and NOMAD [53].

neutrino cross section at energies around $\sim 1 \mathrm{GeV}$ seen by the MiniBooNE experiment [53]. This effective value should account for the enhancement of the total cross sections due nuclear effects in neutrino interaction on nuclei at these energies. Although, different approaches to include nuclear effects makes the cross sections generally smaller than the RFG model.

\section{Transfer Enhancement Model}

Multi-nucleon mechanisms in heavy nucleus have been proposed to explain the enhancement of the total cross section seen by MiniBooNE. Considering the fact that int the MiniBooNE analysis QE interactions are selected by detecting a muon and no pions in the final state, multi-nucleon final states of the form showed in 2.26 are included in the QE sample and their contribution to the total cross section cannot be corrected because they are not included in their Monte Carlo simulation. In these interactions the $\mathrm{W}$ boson is absorbed by two or more correlated nucleons. Electron scattering experiments in Carbon have shown that $\sim 20 \%$ of its nucleons are in a correlated state [55].

$$
\nu[n p] \rightarrow \mu^{-} p p
$$

On the other hand, an enhacement in the transverse electron quasi-elastic response function for nucleons bound in carbon was observed. This effect was parametrized as a function of $Q^{2}$ in terms of a correction to the magnetic form factors of bound nucleons. The authors of this model [56] claim that the parametrization should also be applicable to the transverse cross section in neutrino scattering. If the observed tranverse enhacement is due to multi-nucleon mechanisms; then, from theoy, it is expected that the enhacement in the longitudinal or axial 
contributions is small. The transfer enhancement model is an effective model that accounts for nuclear effects that can be readily incorporated into existing neutrino Monte Carlo generators. One implementation exists in the NuWro [57] neutrino Monte Carlo generator.

\section{Spectral Functions}

An spectral function is the probability distribution of finding a nucleon in the target nucleus with a given value for its 3-momentum and binding energy. Spectral functions for several nucleus have been calculated using electron scattering data and theory predictions. The RFG is a particular simple spectral function where one value of the 3-momentum and binding energy are given a maximum probability.

\subsubsection{Resonant single pion production (RES)}

Neutrinos can excite the target nucleon to a resonance state $\left(N^{*}\right)$,

$$
\nu N \rightarrow \mu^{-} N^{*}
$$

The most important resulting baryonic resonance is the $\Delta(1232)$ resonance and it can decay to a variety of possible final states producing combinations of nucleons and mesons. Nevertheless, the most common final state is the one with a single pion,

$$
N^{*} \rightarrow \pi N^{\prime}
$$

In neutrino and anti-nuetrino scattering off of free nucleons, there are twelve possible resonant single pion reaction channels: six charged current (equations 2.29 trough 2.31) and eight neutral current (equations 2.32 trough 2.35).

$$
\begin{array}{cc}
\nu_{\mu} p \rightarrow \mu^{-} p \pi^{+}, & \bar{\nu}_{\mu} p \rightarrow \mu^{+} p \pi^{-} \\
\nu_{\mu} n \rightarrow \mu^{-} p \pi^{0}, & \bar{\nu}_{\mu} p \rightarrow \mu^{+} n \pi^{0} \\
\nu_{\mu} n \rightarrow \mu^{-} n \pi^{+}, & \bar{\nu}_{\mu} n \rightarrow \mu^{+} n \pi^{-} \\
\nu_{\mu} p \rightarrow \nu_{\mu} p \pi^{0}, & \bar{\nu}_{\mu} p \rightarrow \bar{\nu}_{\mu} p \pi^{0} \\
\nu_{\mu} p \rightarrow \nu_{\mu} n \pi^{+}, & \bar{\nu}_{\mu} p \rightarrow \bar{\nu}_{\mu} n \pi^{+}
\end{array}
$$




$$
\begin{array}{ll}
\nu_{\mu} n \rightarrow \nu_{\mu} n \pi^{0}, & \bar{\nu}_{\mu} n \rightarrow \bar{\nu}_{\mu} n \pi^{0} \\
\nu_{\mu} n \rightarrow \nu_{\mu} p \pi^{-}, & \bar{\nu}_{\mu} n \rightarrow \bar{\nu}_{\mu} p \pi^{-}
\end{array}
$$

Improved measurements and predictions of neutrino-induced single pion production have become important because of the role such processes play in the interpretation of neutrino oscillation data. The Rein and Sehgal model [58] is the most commonly used by neutrino experiment for calculations. This model is based on the quark resonance model and includes contributions for more than 18 resonances in the region $W<2 \mathrm{GeV}$ and gives predictions for both CC and NC resonance production and a prescription for handling interferences between overlapping resonances.

\subsubsection{Deep inelastic scattering (DIS)}

Given enough energy, the neutrino can resolve and scatters off one individual quark in the nucleon, via the exchange of a virtual $\mathrm{W}$ or $\mathrm{Z}$ boson, to produce a lepton; the quark is then ejected and hadronizes. This process is called DIS and it manifests with the creation of a hadronic shower, as a consequence fo the quark hadronization, which can form, among other things, one or more pions. Neutral current (NC) and charged current (CC) channels are possible and are showed in equations 2.36 and 2.37 .

$$
\begin{aligned}
& \nu_{\mu} N \rightarrow \mu^{-} X, \quad \bar{\nu}_{\mu} N \rightarrow \mu^{+} X \\
& \nu_{\mu} N \rightarrow \nu_{\mu} X, \quad \bar{\nu}_{\mu} N \rightarrow \bar{\nu}_{\mu} X
\end{aligned}
$$

Cross sections for DIS are parametrized in terms of the structure functions $F_{1}, F_{2}$ and $F_{3}$,

$$
\begin{aligned}
\frac{d^{2} \sigma^{\nu, \bar{\nu}}}{d x d y}= & \frac{G_{F}^{2} M_{N} E_{\nu}}{\pi\left(1+Q^{2} / M_{W, Z}^{2}\right)^{2}} \\
& {\left[\frac{y^{2}}{2} 2 x F_{1}\left(x, Q^{2}\right)+\left(1-y-\frac{x y M_{N}}{2 E}\right) F_{2}\left(x, Q^{2}\right) \pm y\left(1-\frac{y}{2}\right) x F_{3}\left(x, Q^{2}\right)\right] }
\end{aligned}
$$

Where,

- $x=Q^{2} / 2 M_{N} E_{\nu} y$ is the Bjorken scaling variable,

- $y=E_{\text {had }} / E_{\nu}$ is the inelasticity,

- $E_{\text {had }}$ is the energy in the hadronic system, 
- $M_{N}$ is the nucleon mass,

- $E_{\nu}$ is the incident neutrino enegy,

- $M_{W, Z}$ is the the mass of the $W^{ \pm}$or $Z^{0}$ boson.

- $G_{F}$ is the Fermi coupling constant,

- $Q^{2}$ is the square of the lepton momemtum transfer and

- the $+(-)$ sign in the last term refers to neutrinos (anti-neutrinos).

Structure functions are dimensionless parameters that contain the inner structure of the nucleon and are related by the quark model to the parton distribution functions (PDFs), which describe how partons are distributed in hadrons. The study of DIS plays an important role in the understanding of the PDFs. Using neutrinos for DIS studies is important because only neutrinos can resolve the flavors of the quarks in the nucleus. Indeed, according to the Standard Model, $\nu$ interact with quarks $d, s, \bar{c}$ and $\bar{\nu}$ interact with quarks $u, c \bar{d}$ e $\bar{s}$.

In the GENIE Monte Carlo neutrino generator, for the structure functions, the GRV98 LO [59] parameters are used and the modifications suggested by Bodek and Yang [60] to describe scattering at low $Q^{2}$ are considered.

\subsubsection{Final state interactions (FSI)}

Final state particles produced in the neutrino interaction may interact on their way out of the nucleus, changing the observable signature of the primary interaction. The particles highly subject to these interactions are hadrons with a much less impact on leptons. Among the possible interactions than can occur inside the nucleus are: scattering of final state particles, that modifies its kinematics or eject other particles; pion absortion, where the particle is absorbed inside the nucleus $\left(\pi^{+} n \rightarrow p\right)$; and charge exchange, where the charge of a particle is changed after the interaction $\left(\pi^{+} n \rightarrow \pi^{0} p\right)$. For example, a QE interaction, with a proton in the final state, can emit out of the nucleus several nucleons (this is a different process from the multi-nucleon emission due to correlated nucleon in the nucleus described in 2.5.1) or a RES interaction can mimic a QE because the emitted pion was absorbed in the nucleus due to FSI. In particular, pion absortion is a significant background for QE analysis and only Monte Carlo simulations are able to substract them since detectors are insensitive to what happens inside the nucleus.

In Monte Carlo neutrino event generators, FSI are often simulated using simple and empirical data-driven models like INTRANUKE's hA FSI Model in GENIE [48]. External data on total cross section for each possible nuclear process for pions and nucleons are used. However, 
cascade models where the simulation is done by calculating the interaction probabilty for the possible processes in small steps until the particle leaves the nucleus are also used. 


\section{Chapter 3}

\section{MINER $\nu$ A Experiment}

\subsection{The NuMI beamline}

The Fermilab NuMI beamline provides a high intensity $\nu_{\mu}$ and $\bar{\nu}_{\mu}$ beam for several experiments like MINOS and MINER $\nu \mathrm{A}$ and $\mathrm{NO} \nu \mathrm{A}$. NuMI neutrinos are the final decay product of charged mesons, most pions and kaons, generated by the collision of $120 \mathrm{GeV}$ protons extracted from the Fermilab Main Injector, with a graphite target. Figure 3.1 shows NuMI's main parts and components. A detailed description can be found at [62] and [63].

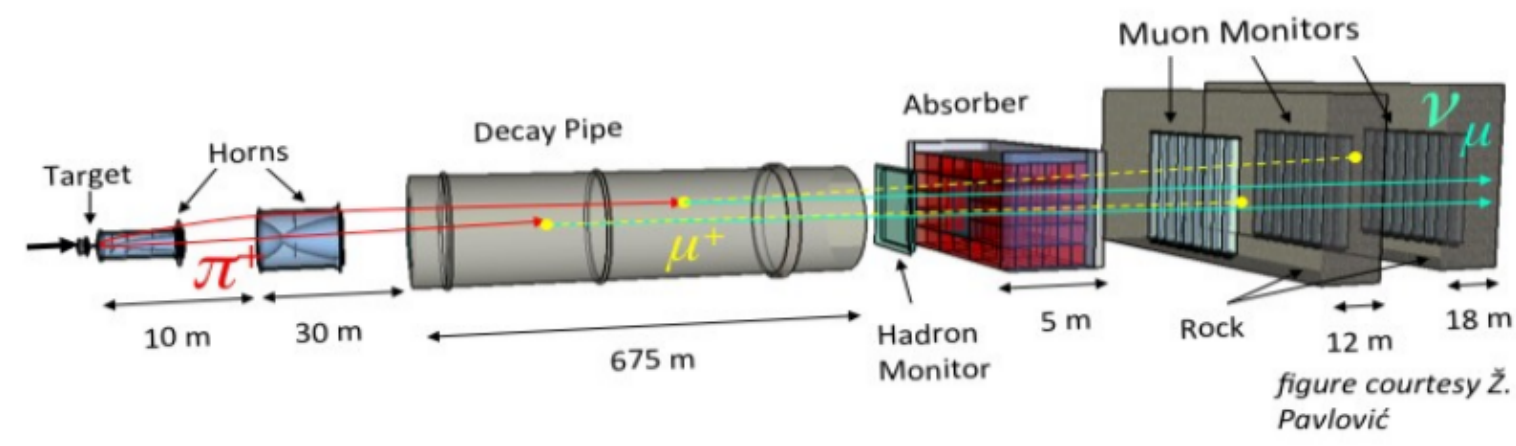

Figure 3.1: NuMI beamline components.

Protons go through several stages before achieving $120 \mathrm{GeV}$ : the Cockcroft-Walton generator, the LINAC, the booster and the Main Injector. The LINAC accelerates the protons up to $400 \mathrm{MeV}$ and sends them to the booster that accelerates them up to $8 \mathrm{GeV}$. At the final stage, the Main Injector takes the protons to the final $120 \mathrm{GeV}$. Every $1.9 \mathrm{~s}$ a $9.7 \mu s$ spill with about $2.5 \times 10^{13}$ protons are extracted and sent towards a $0.95 \mathrm{~m}$ long segmented water cooled graphite target. The target represents 0.95 interaction lengths and each segment is $6.4 \mathrm{~mm}$ thick to minimize reabsorption.

Positive mesons are focused by magnetic horns acting as parabolic magnetic lenses that 
create a toroidal field peaking at $3 \mathrm{~T}$. The horns are water cooled and operated by a pulsed 200 $\mathrm{kA}$ current [63]. After the horns pions and kaons travel through a vacuum region where they decay producing a $\mu$ and a $\nu_{\mu}$. The decay area is a $675 \mathrm{~m}$ long $2 \mathrm{~m}$ diameter cylinder kept at a residual pressure of about 1 Torr or less. Hadrons still present at this stage are stopped at a beam absorber consisting of a water cooled aluminum core surrounded by a steel block and an external concrete chamber. The resulting neutrino beam consists of $97.8 \% \nu_{\mu}$, a few $\bar{\nu}_{\mu}(1.8 \%)$, $\nu_{e}$ and $\bar{\nu}_{e}(0.4 \%)$; the last two being the result of the decay of $\mu^{ \pm}$.

Figure 3.2 shows the possible energy configurations of the NuMI beam: low energy (LE) and medium energy (ME). Different energies are achieved by changing the distance between the target and the second horn in a movement similar to the lenses of an optical system ${ }^{1}$. Pions and kaons of different momenta are selected and focused in the decay region resulting in different energy spectra.

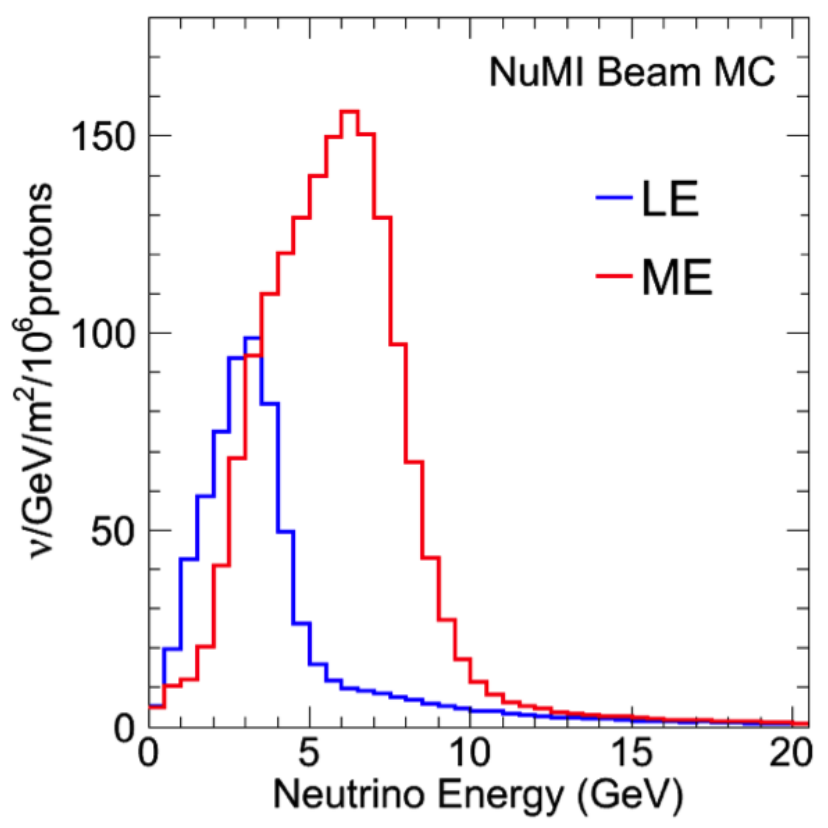

Figure 3.2: NuMI configurations. Low Energy and Medium Energy, plot generated with FLUKA [64].

Changing the horns polarization makes mesons of the opposite signal to be focused so the NuMI can have a beam of neutrinos or antineutrinos. NuMI provides an intense beam for the MINOS experiment whose near detector is housed in an experimental hall $100 \mathrm{~m}$ underground at FERMILAB grounds. MINER $\nu$ A detector is placed just upstream the MINOS near detector.

\footnotetext{
${ }^{1}$ The target is assembled on a system of rails that allows moving the target for a distance of $2.5 \mathrm{~m}$.
} 


\subsection{The MINER $\nu \mathrm{A}$ detector}

MINER $\nu \mathrm{A}$ main objective is to study neutrino scattering with matter with high statistics. MINER $\nu \mathrm{A}$ must be able to:

- identify muons and measure their momenta with precision;

- identify hadrons and $\pi^{0}$ and measure their momenta;

- measure hadronic and electromagnetic showers;

- distinguish neutral current and charged current interactions.

Figure 3.3 shows the MINER $\nu \mathrm{A}$ detector in the experimental hall $100 \mathrm{~m}$ underground. We present here a brief description of the MINER $\nu \mathrm{A}$ detector. A comprehensive and detailed description can be found in [65].

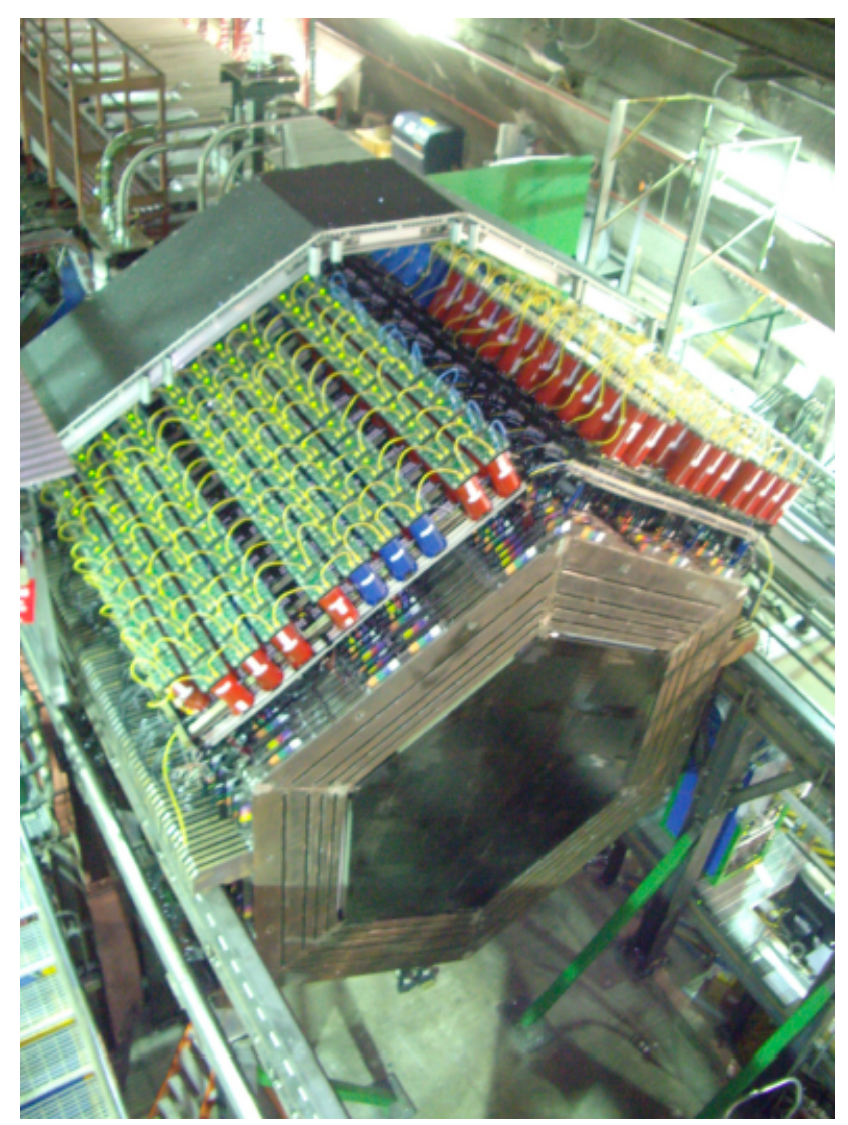

Figure 3.3: Top view of the MINER $\nu \mathrm{A}$ detector.

The MINER $\nu \mathrm{A}$ detector, shown schematically in Figure 3.4, consists basically of two subdetectors: the Inner Detector and the Outer Detector. The Inner Detector itself is subdivided in four subdetectors: 
- Nuclear targets;

- Active target;

- Electromagnetic calorimeter (ECAL);

- Hadronic calorimeter (HCAL).

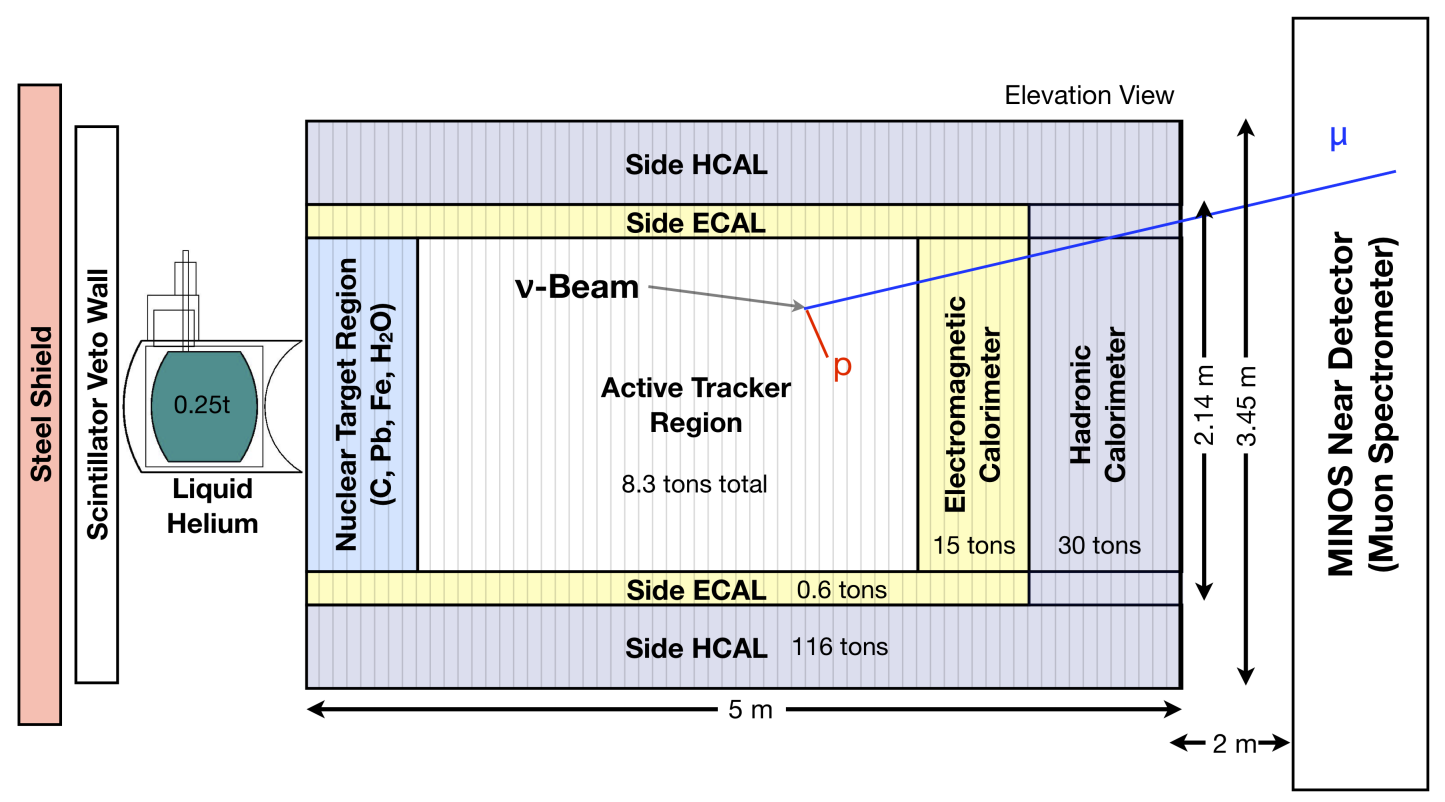

Figure 3.4: Schematic view of the MINER $\nu \mathrm{A}$ detector.

The active target (the core of the detector) consists of strips of solid scintillators. It is the primary volume where interactions happen and where all the analysis is centered. That includes deep inelastic scattering, photon tracking, detection of protons, particle identification through $\frac{d E}{d x}$ (loss of energy by unit of length). Since scintillators, due to their low density, can not hold the whole event, its volume is surrounded by a sampling detector that constitutes the electromagnetic and hadronic calorimeters. In these detectors scintillating strips are interleaved with absorbers (lead sheets in the electromagnetic calorimeter and steel sheets in the hadronic calorimeter). Upstream the detector a veto wall is used to identify charged particles that traverse the detector.

The detector has the shape of a $5.9 \mathrm{~m}$ hexagonal prism of cross section varying from 3.35 $\mathrm{m}$ to $4.10 \mathrm{~m}$. The total mass of scintillators is 6.4 ton. Nuclear targets consisting of Fe (998 
$\mathrm{kg}), \mathrm{Pb}(1023 \mathrm{~kg}), \mathrm{C}(120 \mathrm{~kg})$, liquid $\mathrm{He}(250 \mathrm{~kg})$ and $\mathrm{H}_{2} \mathrm{O}$ are upstream of the detector ${ }^{2}$. The detector high granularity (see scintillating strip description in section 3.3) assures precise vertex reconstruction. The detector is segmented in scintillating planes (section 3.3) and use the Outer Detector (OD) as a supporting structure.

The Inner Detector (ID) has scintillating planes with strips arranged in three different orientations: $\mathrm{X}, \mathrm{U}$ and $\mathrm{V}$ as shown in figure 3.5. $\mathrm{U}$ and $\mathrm{V}$ planes are rotated $\pm 60^{\circ}$ relative to X. Two scintillating planes XU or XV make a module. This arrangement allows tracking reconstruction. Figure 3.6 illustrates one module of the detector active region (structure of a module is depicted on the right).

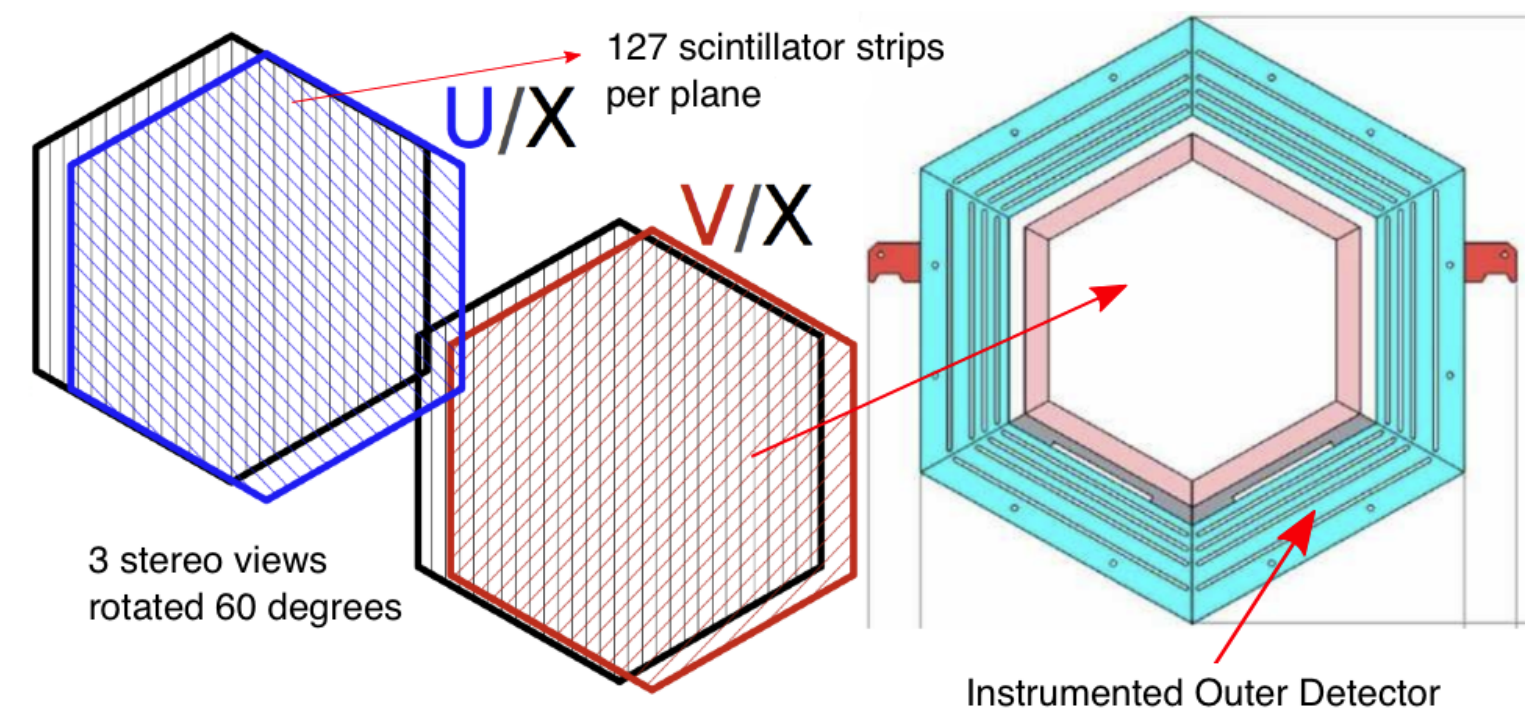

Figure 3.5: Detector active module, featured, $\mathrm{X}, \mathrm{U}$ and $\mathrm{V}$ planes. Note the $\pm 60^{\circ}$ rotation of the planes $\mathrm{U}$ and $\mathrm{V}$ relative to the $\mathrm{X}$ planes.

Figure 3.7 shows a module of the electromagnetic calorimeter. Lead sheets are $0.2 \mathrm{~cm}$ thick and are placed between scintillating planes.

The Inner Detector (ID) is surrounded by a system of absorbers and scintillators that constitute the Outer Detector (OD) (formed by towers arranged at the sides of the hexagon). The downstream part of the detector has a hadronic calorimeter (HCAL) with 1 inch thick absorbers per scintillating plane as shown in Figure 3.8. The electromagnetic calorimeters (ECAL) have $0.2 \mathrm{~cm}$ thick $\mathrm{Pb}$ sheets as absorbers. The ECAL high granularity assures a good resolution for the energy of electrons and photons and make it possible to determine their direction.

The nuclear target region (Figure 3.4) has absorbers placed between active targets making it possible the study of events in different nuclear targets.

\footnotetext{
${ }^{2}$ Considering a transversal section with radius $=90 \mathrm{~cm}$.
} 


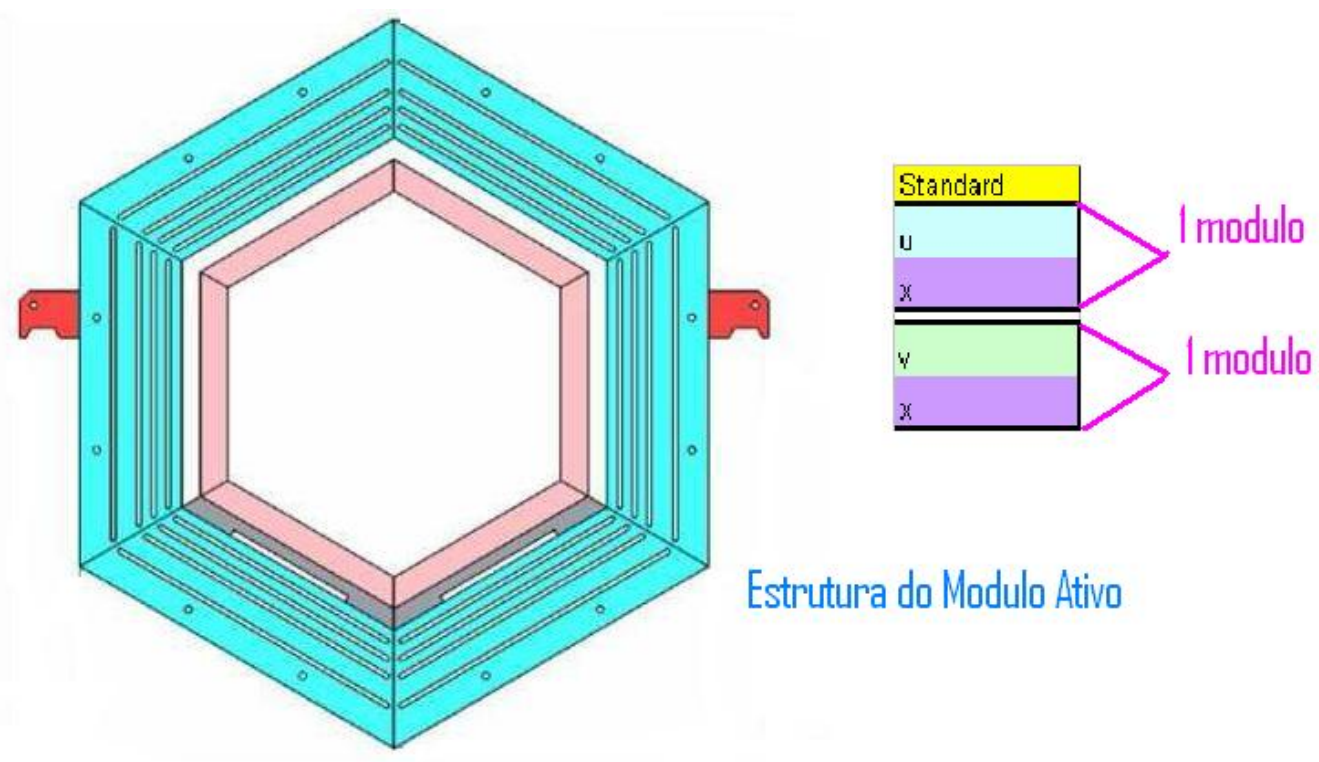

Figure 3.6: Detector ative module. Structure of a module is depicted on the right.

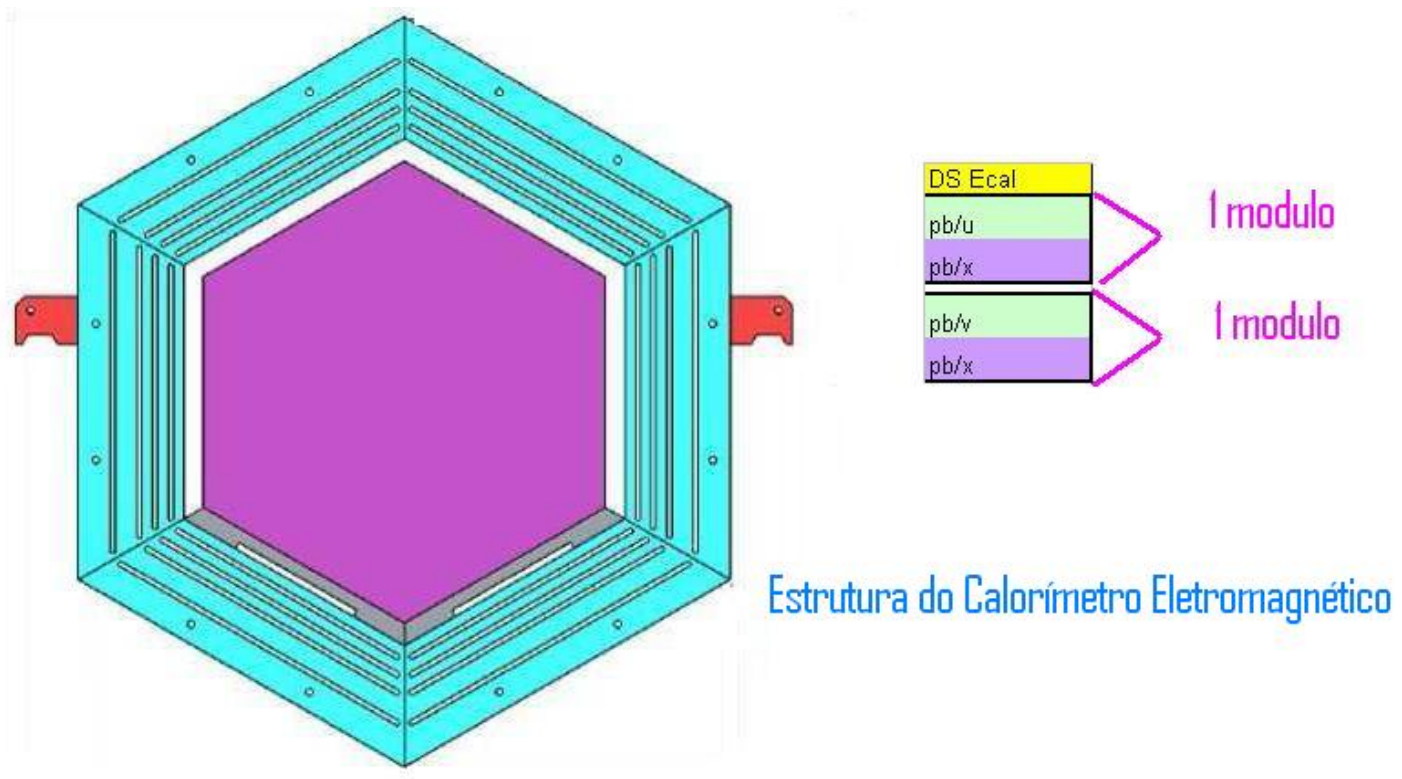

Figure 3.7: Module of the electromagnetic calorimeter. Structure of modules is depicted on the right.

\subsection{The scintillating strips}

The active part of the MINER $\nu \mathrm{A}$ detector is built with triangular prisms of solid scintillator (polystyrene, Dow 663) doped with POP (1\% per weight) and POPOP (0.03\% per weight)) 

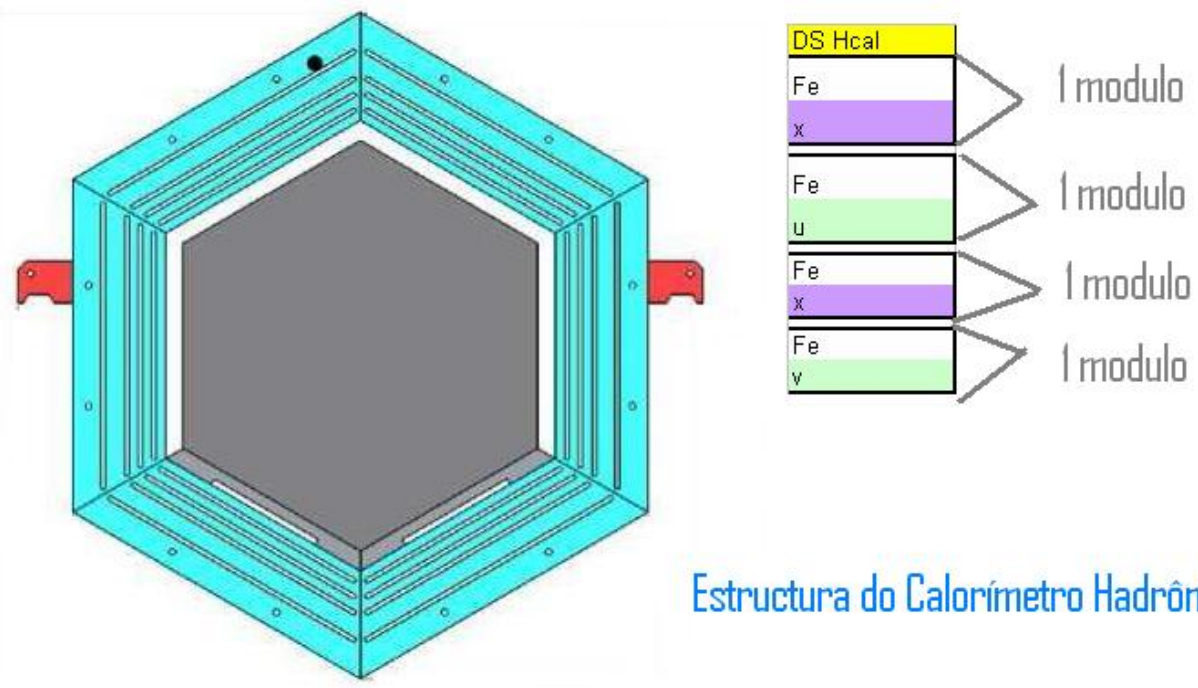

Estructura do Calorímetro Hadrônico

Figure 3.8: Module of the hadronic calorimeter. Structure of the modules with alternating Fe and scintillating planes is depicted on the right.

coated by a reflective layer of $\mathrm{TiO}_{2}$ and traversed by a $1.2 \mathrm{~mm}$ WLS optical fiber (Kuraray Y11 doped at $175 \mathrm{ppm}$ ) as shown in Figure 3.9. The WLS fibers go to optical connectors in both ends of the modules from where clear fibers guide the light to multianode photomultipliers.

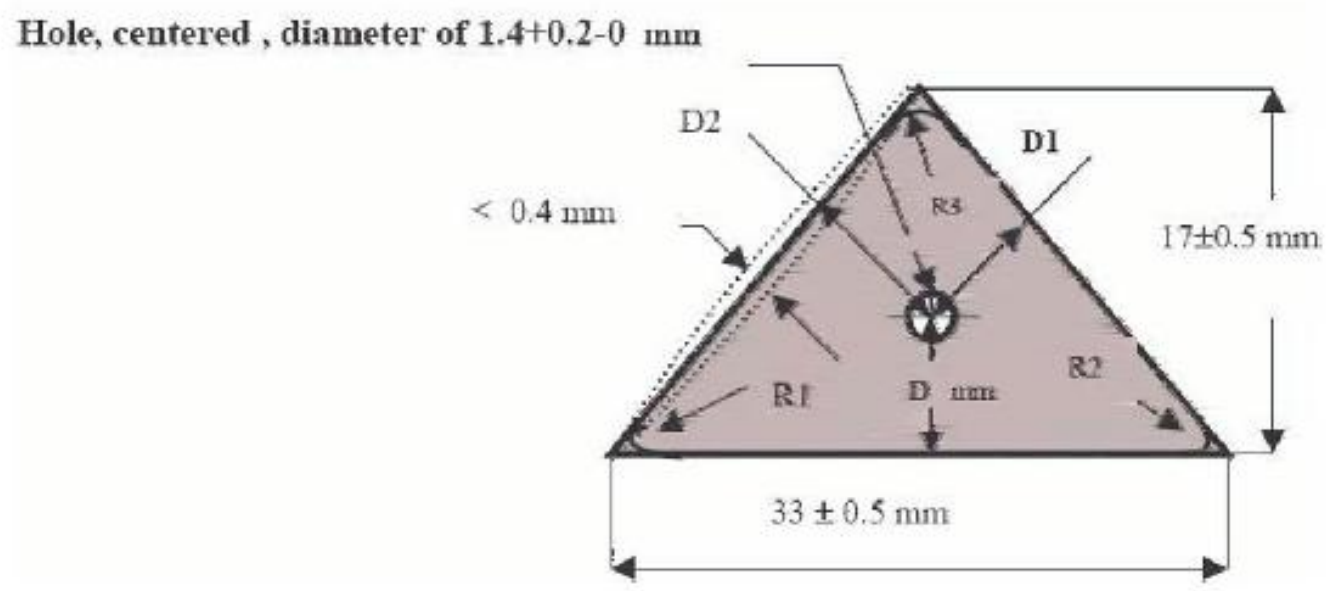

Figure 3.9: Transversal cut of the triangular scintillating prism used in the Inner Detector.

To improve coordinate resolution these triangular elements are assembled in planes (Figure 3.10). Interpolation of the charge split between neighbor scintillating strips allows the determination of the coordinate. 


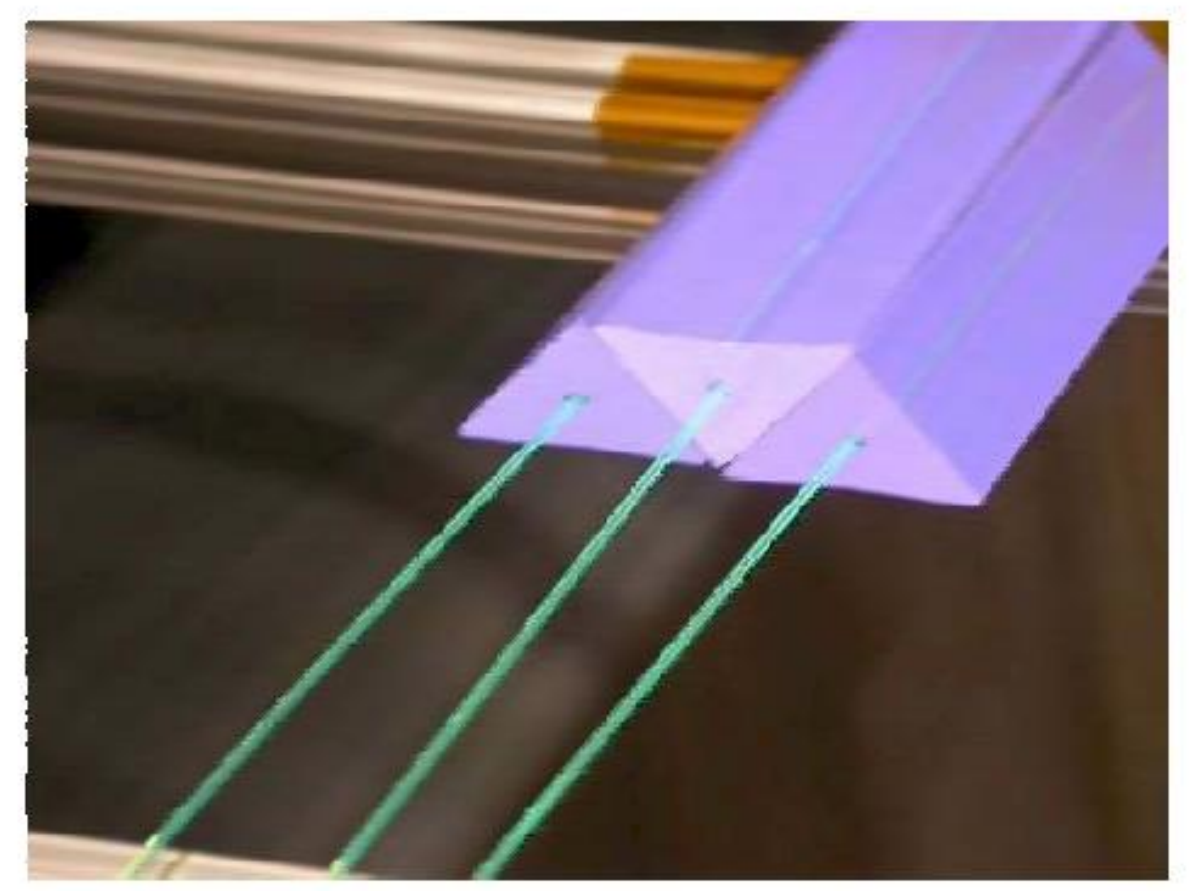

Figure 3.10: Scintillating prisms arranged to form a plane. Each prism holds an optical fiber along its full length.

\subsection{Photodevices}

The light collected in the scintillators must be converted into electric pulses whose characteristics represent the deposited energy. The light signal is strong enough for photodevices with $15 \%$ quantum efficiency. MINER $\nu \mathrm{A}$ detector employs 64 channel multianode photomultiplier R7600U-00-M64 manufactured by Photonics [66].

\subsection{Calorimeters}

MINER $\nu \mathrm{A}$ measures the energy of charged particles $\left(p, \pi^{ \pm}, K^{ \pm}, \mu^{ \pm}\right)$and neutral particles $\left(\pi^{0}, K^{0}, \gamma\right)$ with energies in the order of few $\mathrm{GeV}$ by means of two systems of calorimeters: a set of alternated lead and scintillator planes downstream of the active target for electromagnetic calorimetry and a set of alternated steel and scintillator planes downstream of the active target for hadronic calorimetry; a set of lead, steel, carbon and scintillator blocks assembled around the active target for both electromagnetic and hadronic calorimetry. 


\subsubsection{Electromagnetic calorimeter}

High energy photons are detected by means of the production of pairs of charged particles (bremsstrahlung) that give rise to a shower of $e^{+}, e^{-}$and $\gamma$. Since pair production cross section is proportional to $Z^{2}$, lead sheets are commonly used to produce showers of reasonable size. The typical length of the shower varies with the energy; however, for photons of a few $\mathrm{GeV}$, as the ones we expect in our experiment, $99 \%$ of the energy will stay in $4 \mathrm{~cm} \mathrm{of} \mathrm{Pb}$ ( 7 radiation lengths).

The electromagnetic calorimeter downstream of the active target is made of 20 layers of $\mathrm{Pb}$ (2 mm thick each) alternated with scintillating planes formed by the triangular scintillating prism of scintillator described in 3.3. The expected energy resolution is $6 \% / \sqrt{E}$ where $E$ is given in $\mathrm{GeV}$. The side electromagnetic calorimeter is also made of $2 \mathrm{~mm}$ thick layers of $\mathrm{Pb}$ alternated with layers of scintillator. Photons penetrating the side electromagnetic calorimeter in an angle up to $25^{\circ}$ relative to the beamline are absorbed. Photons penetrating at higher angles will not be totally absorbed by the electromagnetic side calorimeter and will penetrate the side hadronic calorimeter where the remaining shower will be totally contained.

Since the main objective of the downstream layers of $\mathrm{Pb}, \mathrm{Fe}$ and $\mathrm{C}$ (that are thicker) is to work as a target, the calorimetry is not as efficient in this region as it is in the upstreem modules. The way the targets are positioned presents an interaction length between 5 and 10 to the shower. Since the photons in this direction are of lower energy the showers that initiate in the central region will be totally contained in the detector.

\subsubsection{Hadronic calorimeter}

The downstream hadronic calorimeter is placed just after the electromagnetic calorimeter and is made of 20 layers of Fe (2.54 mm thick each) alternated with scintillating planes. The combined action of $4 \mathrm{~cm}$ of $\mathrm{Pb}$ and $50 \mathrm{~cm}$ of Fe stops muons with energy up to $600 \mathrm{MeV}$ and protons with energy up to $800 \mathrm{MeV}^{3}$. The side hadronic calorimeter has layers of $\mathrm{Fe}$ and scintillator (totaling $43.4 \mathrm{~cm}$ of Fe and $12.5 \mathrm{~cm}$ of scintillator) that is enough to stop $750 \mathrm{MeV}$ protons penetrating at $90^{\circ}$ and $1 \mathrm{GeV}$ protons penetrating at $30^{\circ}$.

The expected energy resolution of the hadronic calorimeter is around $50 \% \sqrt{E}$ for hadrons with energy above $1 \mathrm{GeV}$. For less energetic particles the resolution is expected to be $50 \%$ or less, depending on the energy.

\footnotetext{
${ }^{3}$ Since the interaction length for $\mathrm{Fe}$ is $16 \mathrm{~cm}$ protons and pions of higher energy are likely to be stopped.
} 


\subsection{Nuclear targets}

MINER $\nu \mathrm{A}$ has nuclear targets of $\mathrm{Fe}, \mathrm{C}, \mathrm{Pb}, \mathrm{He}$ and $\mathrm{H}_{2} \mathrm{O}$. MINER $\nu \mathrm{A}$ 's target region was implemented according to the following scheme where each F represents a XU or XV structure (a FF pair is a set XUXV): FF Pb/Fe [target 1] FFFF Pb/Fe [target 2] FFFF Pb/Fe/C [target 3] FFFF Pb[target 4] FFFF Pb/Fe [target 5]. Targets 1 and 2 have $60 \% \mathrm{Fe}$ and $40 \% \mathrm{~Pb}$ totaling $230 \mathrm{~kg}$ of $\mathrm{Fe}$ and $\mathrm{Pb}$ in each target. Target 3 has 50\% C, 30\% Fe and 20\% $\mathrm{Pb}$ totaling $140 \mathrm{~kg}$ of $\mathrm{C}, 110 \mathrm{~kg}$ of $\mathrm{Fe}$ and $110 \mathrm{Kg}$ of $\mathrm{Pb}$. Target 4 is pure $\mathrm{Pb}$ with a total mass of $170 \mathrm{~kg}$. Target 5 has $60 \% \mathrm{Fe}$ and $40 \% \mathrm{~Pb}$ each with $115 \mathrm{~kg}$. The total mass of $\mathrm{Fe}$ and $\mathrm{Pb}$ is, respectively, $685 \mathrm{~kg}$ and $855 \mathrm{~kg}$. A water target of hexagonal shaped (with the same dimensions as the detector) was placed between target 4 and 5. Figure 3.11 shows MINER $\nu$ A's target region and its configuration. An additional He target was installed immediately upstream of the detector as illustrated squematically in figure 3.4
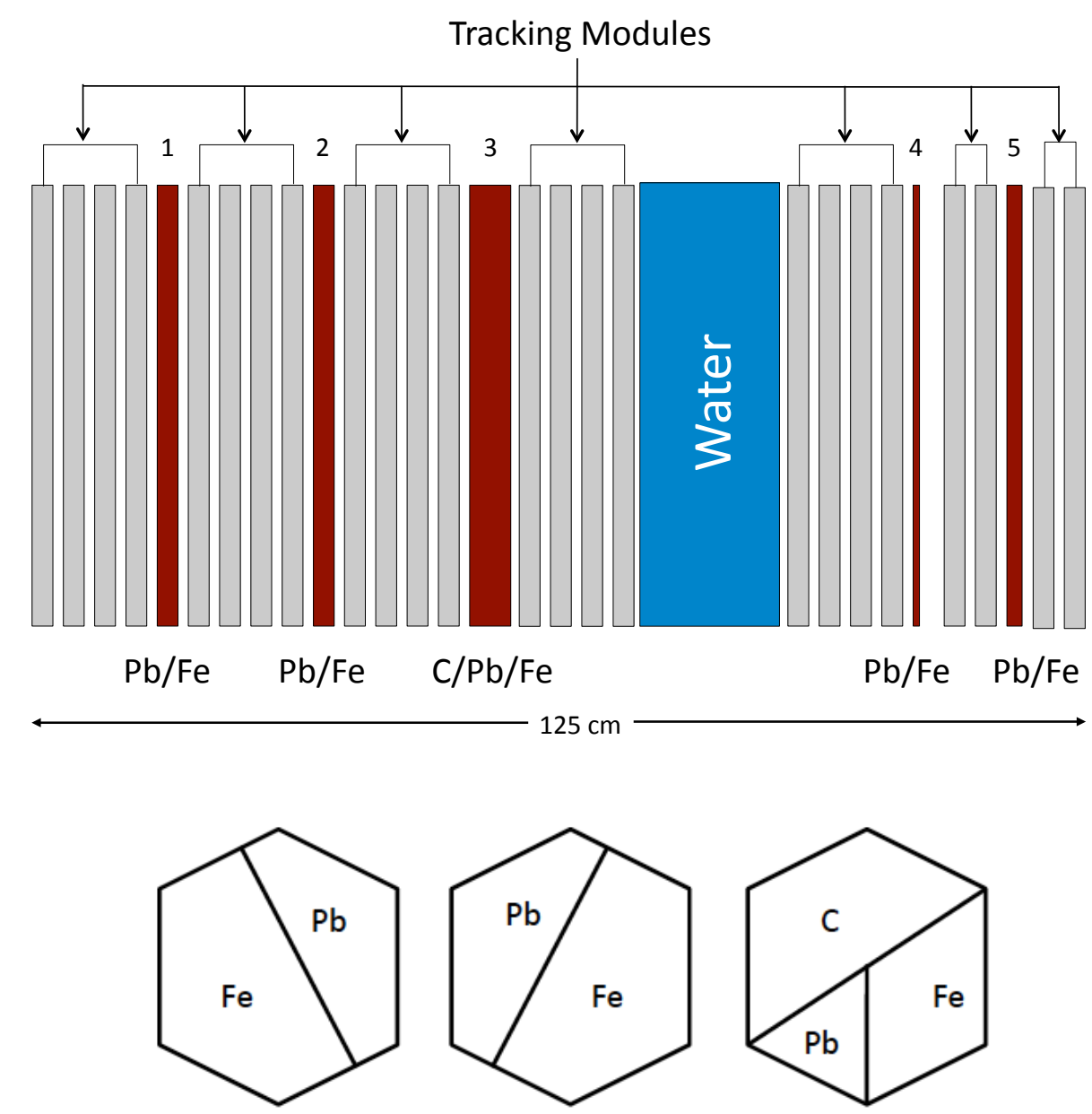

Figure 3.11: Position and configuration of each nuclear target in MINER $\nu \mathrm{A}$ nuclear target region. Figure from [65] 
The total expected number of $\mathrm{CC}$ events for the different nuclear targets is shown in table 3.1.

\begin{tabular}{|c|c|c|}
\hline Target material & Mass (ton) & Charged current sample (K) \\
\hline Helium & 0.25 & 14 \\
Carbon & 0.12 & 9.0 \\
Iron & 0.99 & 54 \\
Lead & 1.02 & 57 \\
water & 0.39 & 20 \\
\hline
\end{tabular}

Table 3.1: Charged current events expected at each nuclear target.

\subsection{Electronic and Data Acquisition (DAQ)}

Table 3.2 summarizes the requirements of the electronics of the MINER $\nu \mathrm{A}$ detector.

\begin{tabular}{|c|c|}
\hline Parameter & value \\
\hline spill & $12 \mu \mathrm{s}$ \\
Repetition time & $>1.9 \mathrm{~s}$ \\
Number of channels & 30,972 \\
Occupation per spill & $2 \%$ \\
gain variation of the photodevice & $4.5 \mathrm{~dB}$ \\
Time resolution & $3 \mathrm{~ns}$ \\
\hline
\end{tabular}

Table 3.2: Some parameters and requirements for the electronics at MINER $\nu \mathrm{A}$.

$\mathrm{MINER} \nu \mathrm{A} \mathrm{DAQ}$ requirements are modest due to the relatively low event rate (about 100 kBytes/s).

Active elements in the MINER $\nu \mathrm{A}$ detector have their signals sent to multi-anode photomultipliers (MAPMT). Information about amplitude and time is digitalized by the electronics and stored for readout by the data acquisition system (DAQ) [67]. Each readout electronic front-end board (FEB) is connected to one single photomultiplier.

Groups of up to $10 \mathrm{FEB}$ are read and the result sent to a crate read-out controller (CROC) housed in a VME crate. Each CROC can accommodate 4 chains of FEB readout. A total of 12 CROCs is needed for the whole MINER $\nu \mathrm{A}$ detector. The VME crates also house a CROC interface module (CRIM), a MINER $\nu$ A timing module (MTM) and a $48 \mathrm{~V}$ power supply. There are no CPU in the VME crates. The DAQ works during the whole spill. After a period of 12 $\mu \mathrm{s}$ the DAQ reads all channels that have a signal above a predefined threshold. Even with a 
high occupancy rate the total number of bytes that are read in each spill is below $200 \mathrm{kB}$ with zero suppression (1 MB without zero suppression).

The photomultipliers are powered by $48 \mathrm{~V}$ power supplies. MINER $\nu \mathrm{A}$ uses the same hardware for data acquisition and for the detector control system (DCS). A single connection is used for the FEB readout and as communication channel for the control of the detector (as, for instance, the control of the MAPMT voltages). The main computers for the DAQ and for the slow control system (the system that controls and monitors the slow varying variables) are close to the VME electronics and are connected to FERMILAB network by two high speed TCP/IP lines. A two CPU server controls the whole system: one CPU dedicated to data acquisition and the other dedicated to control and monitoring. All DAQ machines run on Scientific Linux.

The expected average of data without data suppression is only $100 \mathrm{kB} / \mathrm{s}$ and a two seconds window is available for each $10 \mu$ spill. The highly predicable beam time makes a complex trigger system unnecessary. Instead of such a complex system we simply have a gate signal that opens immediately before the arrival of the beam and all charge an time information from the whole detector is registered just after the end of the spill. The slow control system is also simple with each MAPMT having its own local power supply and with the FEB being in charge of reading the high voltages, temperatures and other parameters used for monitoring and control.

\subsection{Data Collected by the MINER $\nu$ A Experiment}

The MINER $\nu \mathrm{A}$ experiment collected data from November 2009 to April 2012. However, the complete MINER $\nu$ A detector took data from March 2010 to April 2012 since, until March 2010, we had only part of the MINER $\nu$ A detector. MINER $\nu$ A run at NuMi low-energy configuration along with the MINOS experiment. During most of this period, the NuMI beamline switched between its low-energy neutrino and anti-neutrino configuration with a peak energy of $\sim 3 \mathrm{GeV}$. The periods of time when the NuMI beamline works in different configurations are called special runs. Data from the special runs will eventually be analized to constraint the neutrino and antineutrino flux prediction in the MINER $\nu \mathrm{A}$ detector and reduce systematic errors. Figure 3.12 shows the different data sets of data collected by the MINER $\nu \mathrm{A}$ experiment. A very detailed description of the different data sets can be found in chapter 6 of [69]. The total exposure was of $3.98 \times 10^{20}$ POT $^{4}$ (low-energy neutrino beam), $1.7 \times 10^{20}$ POT (low-energy anti-neutrino beam) and $0.49 \times 10^{20} \mathrm{POT}$ (special runs).

In August 2013 the MINER $\nu \mathrm{A}$ experiment started to take data at the NuMI medium-energy configuration with a peak energy of $\sim 8 \mathrm{GeV}$ along with MINOS+ and NO $\nu \mathrm{A}$ experiments.

\footnotetext{
${ }^{4}$ Protons On Target
} 


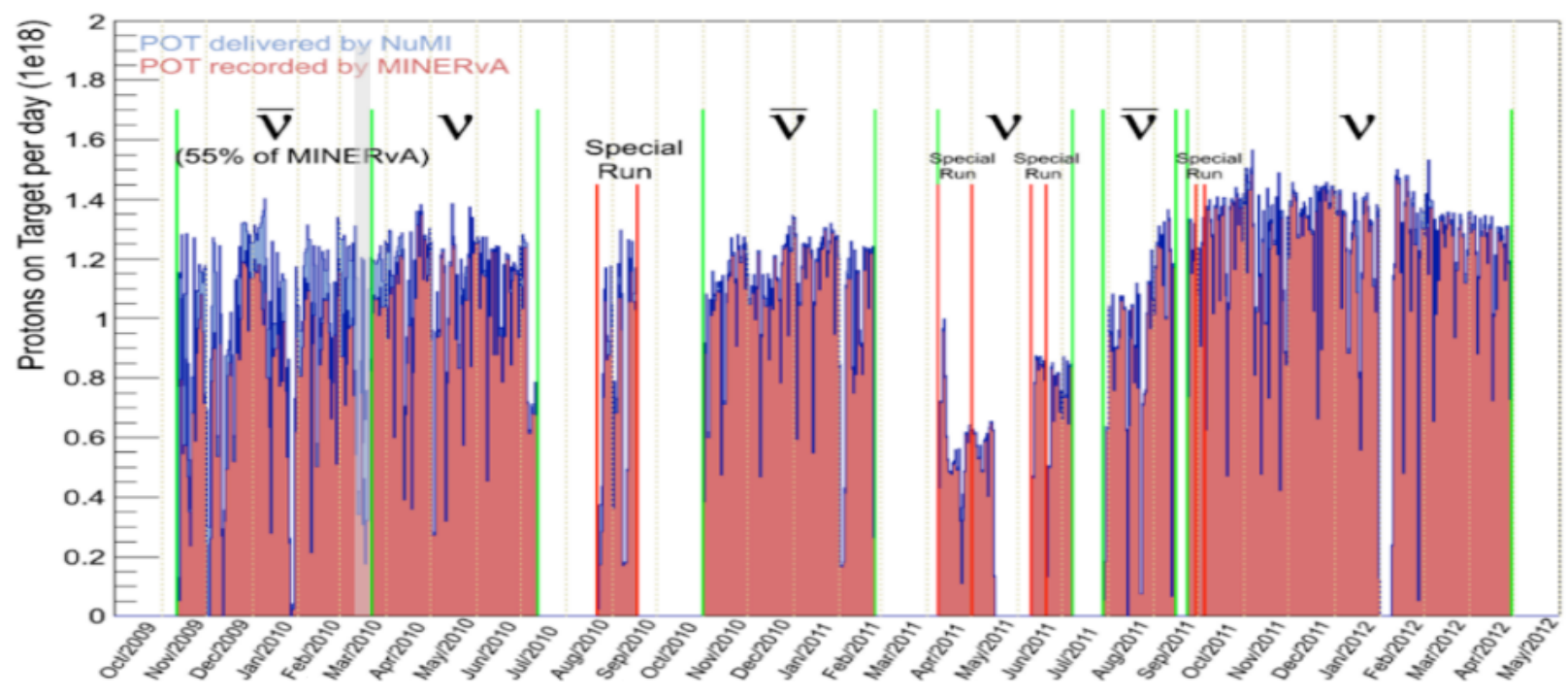

Figure 3.12: The different periods of data taking in the MINER $\nu \mathrm{A}$ experiment during the low energy configuration of the NuMI beamline. 


\section{Chapter 4}

\section{Reconstruction and Event Selection}

\subsection{Introduction}

The objetive of this section is to describe the reconstruction of charged current quasi-elastic (CCQE) interactions on plastic scintillator in the MINER $\nu \mathrm{A}$ detector.

\subsection{Event Sample}

\subsubsection{Data}

The present analysis uses the data collected by the MINER $\nu \mathrm{A}$ detector between March and July 2010 (see figure 3.12 that corresponds to $9.42 \times 10^{19}$ POT in the neutrino low-energy configuration of the NuMI beamline. Notice that this represents $\sim 1 / 4$ of the total data collected in the low-energy neutrino beam configuration. The remaining neutrino data was not ready for analysis (calibrations and quality checks were still missing) at the time of the completion of this thesis. However, as we will see later, the total uncertainty in the result of this analysis is not dominated by statistical errors but systematics errors.

\subsubsection{Monte Carlo}

The Monte Carlo (MC) simulation in the MINER $\nu \mathrm{A}$ experiment is an implementation of two standard software packages commonly used in high-energy neutrino experiments: GENIE [48] and GEANT4 [71]. The GEANT4 software package version 9.2p03 is used to simulate the NuMI beamline. The hadron production from $120 \mathrm{GeV}$ protons at the NuMI target, the magnetic horn system, and the decay of pions and kaons to muons and neutrinos are simulated.

The hadron production simulation is based on the FTFP_BERT physics model in GEANT4, which is the combination of the FTFP model for hadron colliding in a nucleus at energies greater 
than $4 \mathrm{GeV}$ and the Bertini cascade model for energies below $4 \mathrm{GeV}$. The FTFP_BERT model is compared to different hadron production data [72] [73] [74]. As an example, the result of the comparison between FTFP_BERT and data from the CERN NA49 experiment [72] is showed in figure 4.1 where appreciable discrepancies can be seen. Comparisons are made using the invariant production cross section, $f=E d^{3} / d p^{3}$, as a function of the Feynman scaling variable, $x_{F}=2 p_{L} / \sqrt{s}$, and the transverse momentum of the outgoing particle, $p_{T}$. Due to the disagreement, a weight to the events in the FTFP_BERT prediction is calculated to match the hadron production data,

$$
\text { weight }=C(158 G e V \rightarrow 120 G e V) \frac{f\left(x_{F}, p_{T}\right)_{\text {data }}}{f\left(x_{F}, p_{T}\right)_{F T F P}}
$$

where $C$ is the correction factor, calculated with FLUKA [64], needed to scale the proton energy from NA49's hadron production data $(\sim 158 \mathrm{GeV})$ to NUMI's proton energies $(\sim 120 \mathrm{GeV})$. Events in the simulation outside the hadron production data range are not weighted.

A correction to the simulation as a function of neutrino energy is obtained by calculating the ratio between the weighted and unweighted flux. Figure 4.2 shows the calculated correction and figure 4.3 the estimated flux after the correction. The wegihted neutrino flux is the one used in this analysis.

After having simulated the neutrino flux, GENIE is used to generate neutrino interactions in the MINER $\nu \mathrm{A}$ detector using the neutrino flux as an input. It is also used to define the generated signal and background events for this analysis. Signal events in the MC are the ones defined by GENIE as $\nu_{\mu}$ CC QE (excluding events with charm quarks in the final state that GENIE considers QE) with a neutrino energy from $1.5 \mathrm{GeV}$ to $10 \mathrm{GeV}$ in the fiducial volume (defined later) of MINER $\nu$ A's tracker region. Background events are all other interaction generated by GENIE; they can be split in: CC RES (resonant pion production), CC DIS (deep inelastic scattering) and Other. This last category contains the remaining types of interactions such as, but not limited to, coherent pion production, anti-neutrino interactions (wrong sign events) and neutral current events. The theoretical models and parametrizations used by GENIE to define these categories of interactions, as well as FSI, are described in section 2.5. The GEANT4 software package version 9.4.p02 is then used to simulate the passage of final state particles, generated by GENIE, through the MINER $\nu \mathrm{A}$ detector. It also simulates the optical system and electronics used for readout of the scintillator strips.

The MC simulation generates only one interaction in the MINER $\nu \mathrm{A}$ detector for each simulated gate. However, real gates from the data has more activity coming from several interactions inside or outside the MINER $\nu \mathrm{A}$ detector. This additional activity is introduced in the MC by overlying a random data gate in the simulated gate. In this way, effects due to the overlap or proximity in time of two or more interaction can be simulated. The most important effect 


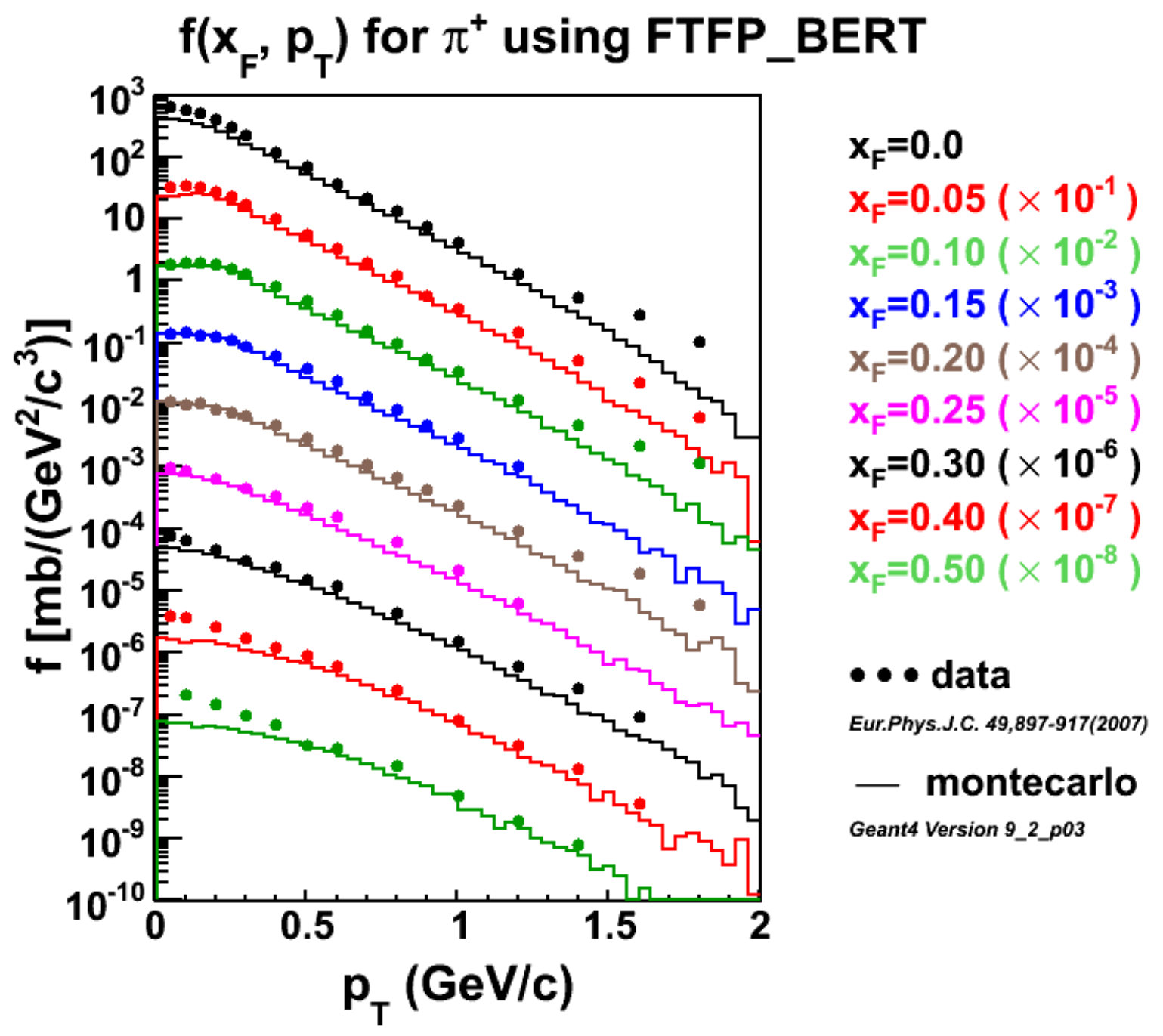

Figure 4.1: Comparison of the invariant production cross section between the FTFP_BERT model in GEANT4 and CERN NA49 hadron production data as a function of the Feynman scaling variable and the transverse momentum of the outgoing particle.

is the one due to detector dead-time which is the time after one interaction that the detector needs in order to detect the next interaction.

In order to be able to compare a distribution made with data events and its equivalent in the MC simulation, the MC sample needs to be scaled. We do this by using two kinds of normalization factors: the absolute normalization factor, $M C_{A b s}^{N o r m}$, and the area normalization factor, $M C_{\text {Area }}^{N o r m}$. They are calculated according to the following formulas,

$$
\begin{gathered}
M C_{\text {Abs }}^{\text {Norm }}=\frac{\text { POT }_{\text {data }}}{P O T_{M C}} \\
M C_{\text {Area }}^{\text {Norm }}=\frac{\# \text { Event }_{\text {data }}}{\# \text { Events }_{M C}}
\end{gathered}
$$

Where: 


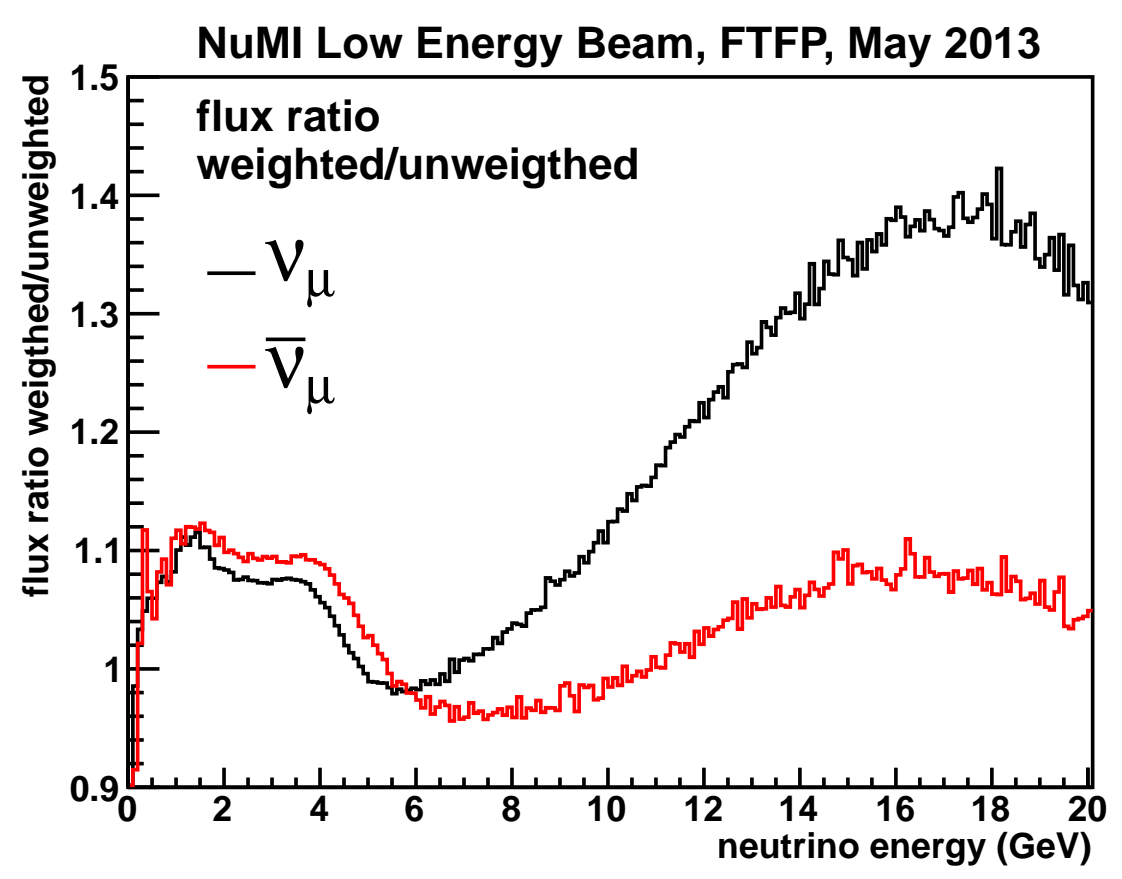

Figure 4.2: Correction to the neutrino and anti-neutrino flux in the MINER $\nu \mathrm{A}$ detector calculated as the ratio of the flux after and before applying the weights from the hadron production data comparisons. $\nu_{\mu}$ refers to the muon neutrino flux when NuMI's magents are focusing positive mesons and $\bar{\nu}_{\mu}$ to the muon anti-neutrino flux when focusing negative mesons.

- $P O T_{d a t a}$ and $P O T_{M C}$ are the POT exposure used to generated the data and MC samples rescpectively.

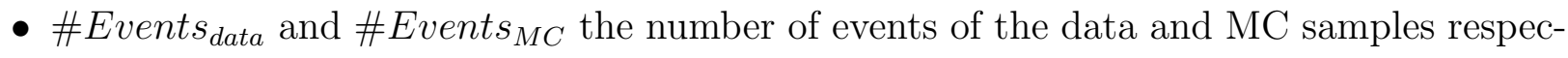
tively.

The amount of MC generated events that is used in our analysis corresponds to $9.26 \times$ $10^{20} \mathrm{POT}$ so the absolute normalization factor is 0.101634 . The area normalization factor is calculated for each distribution since it depends of the number of events used to make that distribution. These numbers is used to absolute normalize the MC sample before comparing it with data.

\subsection{Event Reconstruction}

Reconstruction of neutrino interactions in the MINER $\nu \mathrm{A}$ detector starts with binary data received from the data adquisition system [67]. This binary data represents the activity in the 


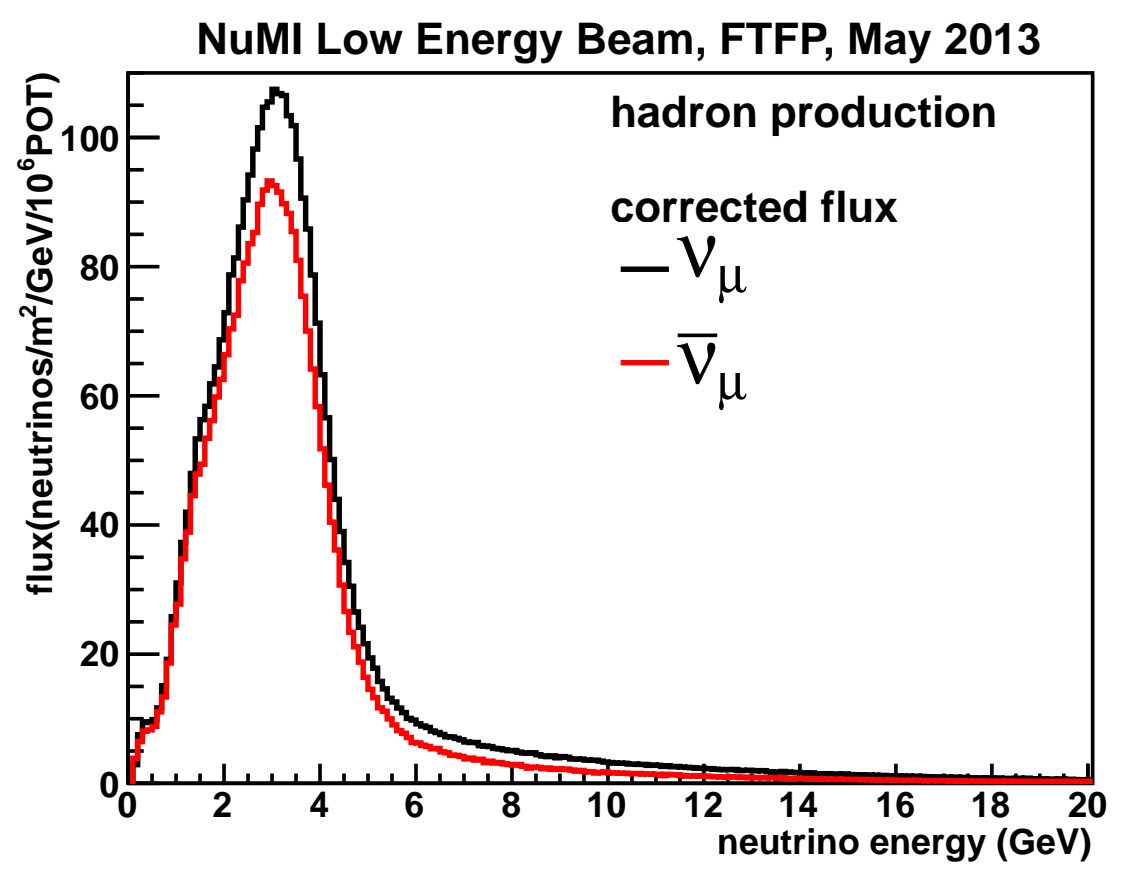

Figure 4.3: Corrected flux as a function of neutrino energy used in the analysis. $\nu_{\mu}$ refers to the muon neutrino flux when NuMI's magnets are focusing positive mesons and $\bar{\nu}_{\mu}$ to the muon anti-neutrino flux when focusing negative mesons.

detector during one gate ${ }^{1}$. The information contained in the binary data is converted into more useful objects in MINER $\nu$ A's reconstruction software that represent each active scintillator strip in the detector. These objects are called hits and are subjected to several calibrations whose details are described in [65]. After time, position and energy calibrations, hits can be grouped as shown in Figure 4.4. Each group of hits represents one event.

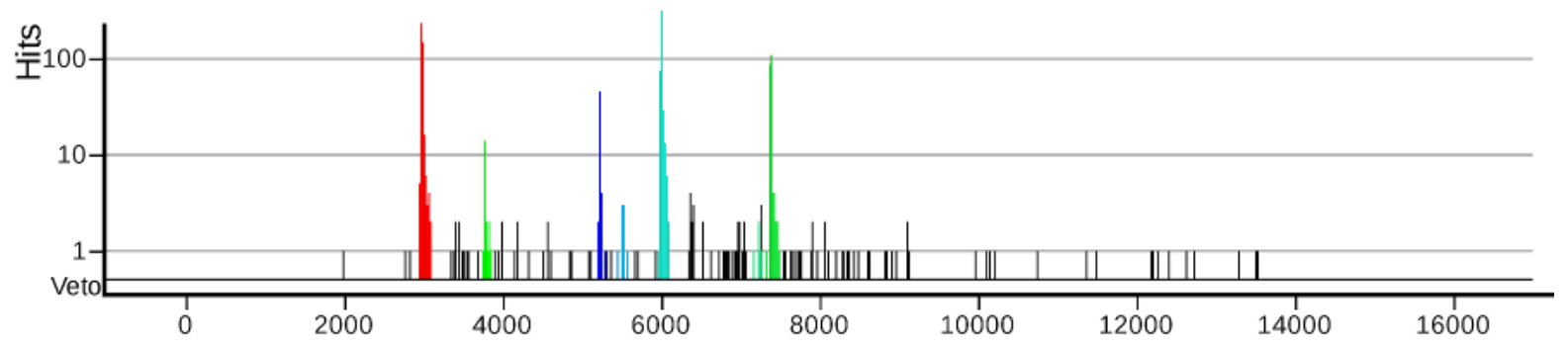

Figure 4.4: The time distribution of hits in the MINER $\nu$ A detector during a NuMI beam spill. The different colored peaks are identified as events that represent a neutrino interaction.

As mentioned in chapter 3, strips have a triangular cross section so a particle passing through

\footnotetext{
${ }^{1}$ This is the $16 \mathrm{~ns}$ time window where the detector is active and the $9.72 \mu \mathrm{s}$ NuMI spill is contained.
} 
a plane deposits its energy in at least two strips. In order to represent the deposition of energy in a plane, adjacents hits in the same plane are grouped to form clusters. More details about this and other types of reconstruction done in the MINER $\nu \mathrm{A}$ detector, as well as the detector performance, can be found in [65], [68], [69] and [70].

We will briefly describe the use of clusters for the reconstruction of muons and recoil energy since they will be used to analyze CCQE interactions.

\subsubsection{Muon Reconstruction}

A long track pattern recognition algorithm based on an implementation of the Kalman filter that incorporates multiple scattering is used to find long (more than 9 modules) tracks in the MINER $\nu \mathrm{A}$ detector. Long tracks found in MINER $\nu \mathrm{A}$ are then projected into the MINOS near detector in an attempt to find a matching MINOS track. If found, both tracks, the one in MINER $\nu \mathrm{A}$ and the one in MINOS, are merged and called a MINOS match track. All MINOS match tracks are assumed to be muons. The interaction's reconstructed vertex is located at the beginning of the muon track. Figure 4.5 shows a charged current event with a fully reconstructed muon using the MINER $\nu \mathrm{A}$ event display [75].

The track curvature in association with the magnetic field produced by MINOS magnetic coil allows the reconstrucion of the muon charge and energy. The energy of muons that stop in the MINOS near detector can also be reconstructed by the range method that uses the BetheBloch equation [37] to calculate the total energy loss during the passage of the muon trough the MINER $\nu \mathrm{A}$ and MINOS detectors. The muon energy resolution is $10 \%$ for muons reconstructed by curvature and $5 \%$ when reconstructed by the range method. The muon reconstruction, as described, is used by all Charged Current (CC) analysis, where a muon is present.

\section{Tracking and Matching Efficiency}

The tracking efficiency of muons generated by interactions inside the tracker region of the MINER $\nu \mathrm{A}$ detector times the MINOS matching efficiency was calculated as a function of the muon momentum measured by MINOS. The procedure consists in select muons with reconstructed charge and momentum starting in MINOS' front face and pointing them back to MINER $\nu \mathrm{A}$ to check if a MINER $\nu \mathrm{A}$ matched track exists. We calculate the efficiency using the formula,

$$
\epsilon_{\text {track }} \times \epsilon_{\text {match }}\left(p_{\text {MINOS }}\right)=\frac{N_{\text {tracks }}^{\text {MINOS MINERDA }}\left(p_{\text {MINOS }}\right)}{N_{\text {tracks }}^{\text {MINOS }}\left(p_{\text {MINOS }}\right)}
$$

Where:

- $\epsilon_{\text {track }} \times \epsilon_{\text {match }}$ is the muon tracking matching efficiency. 

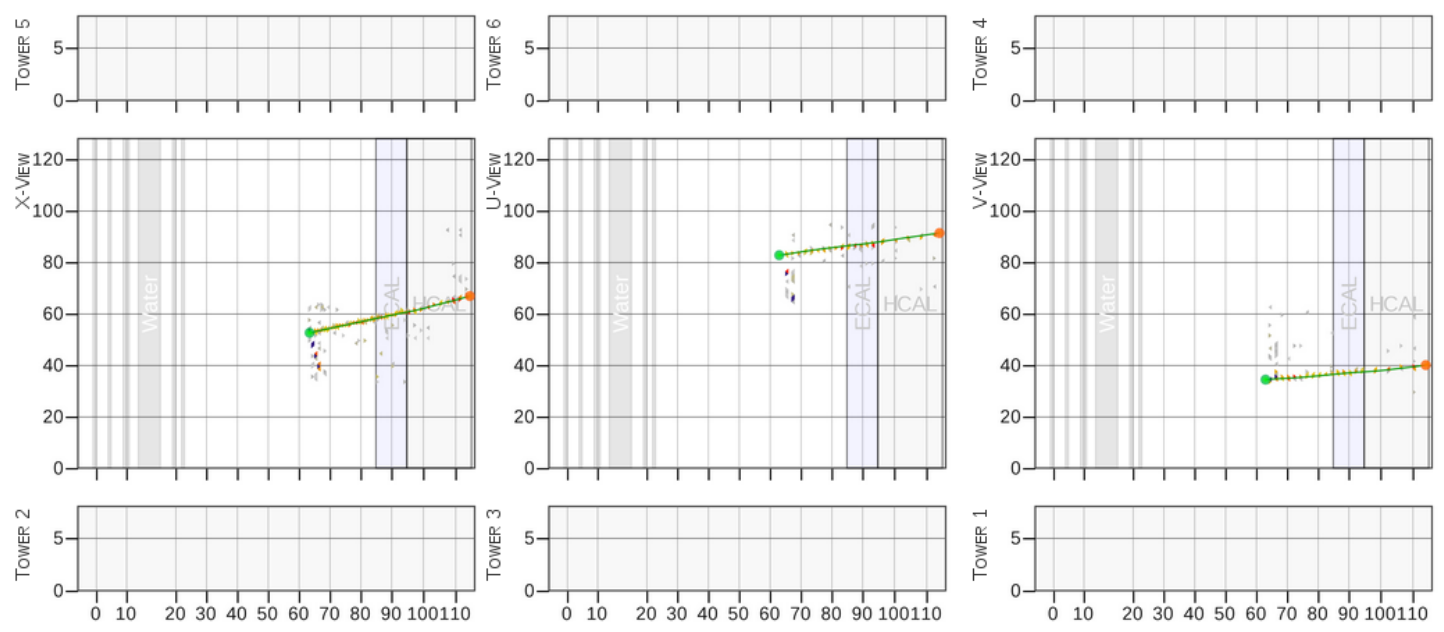

Minos U View

Minos $\vee$ View
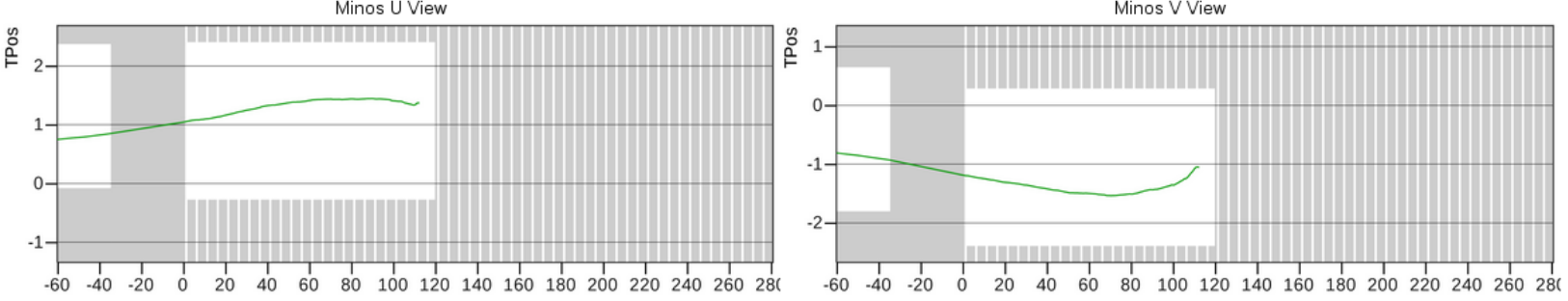

Figure 4.5: The MINER $\nu \mathrm{A}$ event display. Top: a charged current neutrino event in the tracker region with a reconstructed negative muon exiting from the back (reconstructed track is shown as a green line) in the three views of the MINER $\nu \mathrm{A}$ detector. Bottom: the same event whose muon is exiting MINER $\nu \mathrm{A}$ and entering MINOS. This muon has been reconstructed by both detectors and the resulting tracks merged.

- $N_{\text {tracks }}^{M I N O S}$ is the number of selected MINOS tracks.

- $N_{\text {tracks }}^{\text {MINOS,MINER } A A}$ is the number of selected MINOS tracks with match in MINER $\nu \mathrm{A}$.

- $p_{M I N O S}$ is the muon momentum measured by MINOS.

The result is presented in figure 4.6 together with the ratio between data and MC.

The main sources of tracking and matching failures are interaction pile up, high energy showers and both. Interaction pile up complicates the muon reconstruction if two or more interactions are included in the same event. High energy showers can obscure the muon track preventing its reconstruction and can also, together with pile up, generates considerable regions in the detector where it is not possible to detect the muon due to dead time. The weightedaverage ratio between data and MC in figure 4.6 is 0.98 which means that the tracking matching efficiency in MC is $2 \%$ higher than in data. In other words, the failure rate is lower in MC than in data. The source of this discrepancy is not fully understood so the calculated average ratio between data and $\mathrm{MC}$ is used as a correction to the absolute normalization of the MC with 

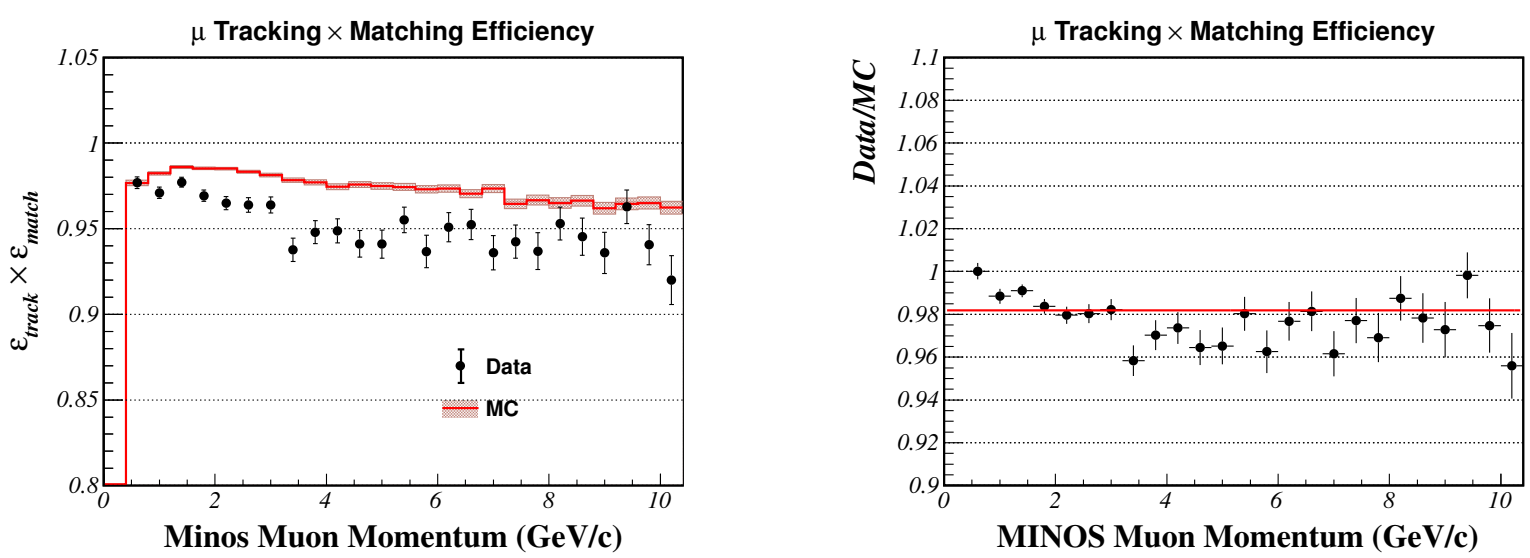

Figure 4.6: Left: The tracking efficiency of muons generated by interactions inside the tracker region of the MINER $\nu \mathrm{A}$ detector times the matching to MINOS efficiency in data and MC as a function of the muon momentum measured by MINOS. Right: The comparison between data and MC of the same efficiency where the horizontal red line represents the average value. Errors in both plots are statistical errors only.

0.01 one-sigma uncertainty. All MC based distribution will contain this correction factor from now on.

\section{Neutrino Energy and $Q^{2}$ reconstruction}

Under the CCQE hypothesis of the interaction, the reconstruction of muon kinematics can be used to infer two important properties of the interaction needed for this analysis; namely, the neutrino energy and the 4-momentum transfer $Q^{2}$. This is possible thanks to the relative simple two body kinematics involved in CCQE interactions. In fact, charged current QE interactions are very useful for neutrino experiments because the neutrino flavor can be identified by the charge of the final state muon and the neutrino energy can be calculated by measuring muon kinematics.

In order to reconstruct the neutrino energy, we assume that the target nucleon is at rest and quasi-free inside the nucleus (RFG aproximation see 2.5.1). By using the conservation of 4-momentum in a two body elastic colision and neglecting the neutrino mass will lead us to our neutrino energy expression for Charged Current QE scattering.

$$
E_{\nu}^{\mathrm{QE}}=\frac{2\left(M_{n}-\mathrm{E}_{\mathrm{B}}\right) E_{\mu}-\left[\left(M_{n}-\mathrm{E}_{\mathrm{B}}\right)^{2}+m_{\mu}^{2}-M_{p}^{2}\right]}{2\left[\left(M_{n}-\mathrm{E}_{\mathrm{B}}\right)-E_{\mu}+\sqrt{E_{\mu}^{2}-m_{\mu}^{2}} \cos \theta_{\mu}\right]}
$$

where $M_{n}, M_{p}$ and $m_{\mu}$ are the neutron, proton and muon masses, $E_{\mu}=T_{\mu}+m_{\mu}$ is the total muon energy, $\theta_{\mu}$ is the angle of the muon track with respect to the neutrino direction, and $\mathrm{E}_{\mathrm{B}}$ is the nuclear binding energy in carbon $\left(\mathrm{E}_{\mathrm{B}}=34 \mathrm{MeV}\right.$ in this analysis). 
The 4-momentum transfer to the target nucleon represented by the relativistic invariant, $Q^{2}=-q^{2}$, where $\mathrm{q}$ is the 4 -momentum of the $W^{ \pm}$boson, can be reconstructed as,

$$
Q_{\mathrm{QE}}^{2}=-m_{\mu}^{2}+2 E_{\nu}^{\mathrm{QE}}\left(E_{\mu}-\sqrt{E_{\mu}^{2}-m_{\mu}^{2}} \cos \theta_{\mu}\right)
$$

Both expressions are going to be used in our main event selection. Notice that these values for the neutrino energy $E_{\nu}^{\mathrm{QE}}$ and 4-momentum transfer $Q_{\mathrm{QE}}^{2}$ are not valid for neither nonCCQE interactions nor interactions where the target correspond to correlated nucleons (multinucleon mechanisims). However, for these interactions, we still can interpret $Q_{\mathrm{QE}}^{2}$ and $E_{\nu}^{\mathrm{QE}}$ as a parametrizations of the muon kinematics. The subindice $Q E$ in both expressions, $E_{\nu}^{\mathrm{QE}}$ and $Q_{\mathrm{QE}}^{2}$, are there to remind us that this formulas are deduced using a CCQE hypothesis.

\subsubsection{Recoil Energy reconstruction}

Clusters that do not belong to the muon track are reconstructed and classified in different ways.

Vertex Energy The energy in clusters within $300 \mathrm{~mm}$ of the reconstructed vertex is summed and called vertex energy. Given the geometry of the detector, the shape of the space over which energy is summed is not a sphere, but rather the intersection of three cylinders of half-length $R=300 \mathrm{~mm}$ at $60^{\circ}$ angles to each other. The $300 \mathrm{~mm}$ radius corresponds to about 6 modules in each direction along the z-axis and about 18 strips in each direction within a plane. It is also the average maximum distance a $\sim 225 \mathrm{MeV}$ proton and a $\sim 225 \mathrm{MeV}$ pion will travel in the scintillator before losing all its energy.

Isolated Blobs Energy The remaining clusters, whose energy is not below $1 \mathrm{MeV}$ and where $\left|t_{\text {cluster }}-t_{\text {muontrack }}\right|<25 \mathrm{~ns}$, are considered to build independent shower-like groups of clusters called isolated blobs. These objects are required to contain clusters in several views so they can be reconstructed in 3-dimensions. The energy of isolated blobs is summed and called isolated blobs energy.

Dispersed Energy All the remaining energy in the tracker and ECAL regions of the inner detector is summed and called dispersed energy. Only clusters within $25 \mathrm{~ns}$ of the muon track time are considered.

Recoil Energy This is just the sum of isolated blobs energy and dispersed energy, $E_{\text {recoil }}=$ $E_{\text {isolated }}+E_{\text {dispersed }}$. It represents the total energy outside the vertex region.

CCQE interactions are expected to have low recoil energy away from the vertex. The recoil energy reconstructed in the way described here will help us to isolated a CCQE sample of neutrino interactions in the MINER $\nu$ A's tracker region. 
An example on how the recoil energy is reconstructed is given in figure 4.7 where an nonCCQE interaction candidate with a considerable amount of recoil is reconstructed.

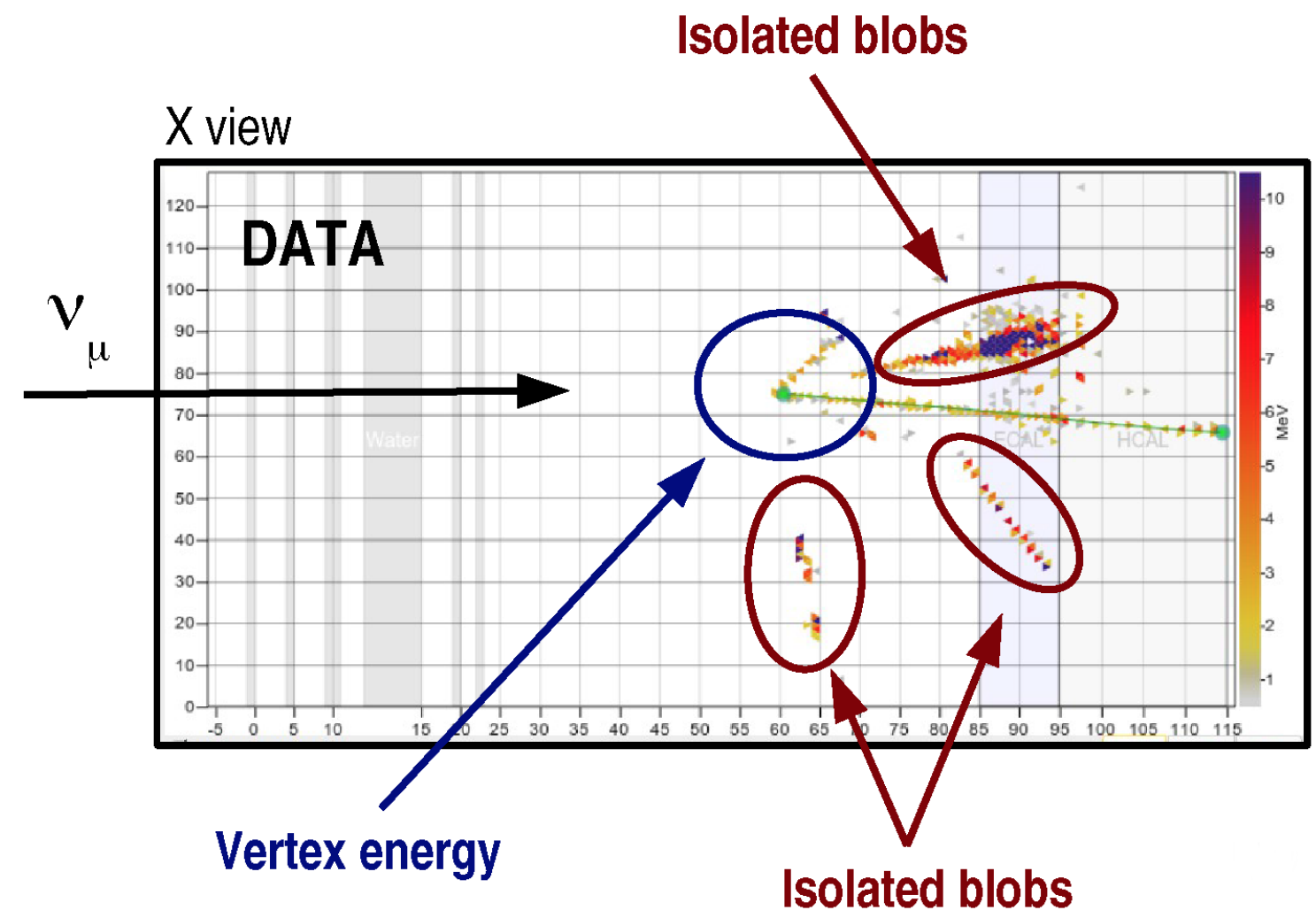

Figure 4.7: The MINER $\nu \mathrm{A}$ event display showing one view (the $\mathrm{X}$ view) of a non-CCQE interaction candidate and the different recoil energy classifications used in its reconstruction

\subsection{Event Selection}

This section describes the selection of CCQE $\left(\nu_{\mu}+n \rightarrow p+\mu^{-}\right)$interactions at energies between $1.5 \mathrm{GeV}$ and $10 \mathrm{GeV}$ with an average of $\sim 3.5 \mathrm{GeV}$ in the tracker region of the MINER $\nu \mathrm{A}$ detector. The signature of CCQE interactions is relatively simple: a proton and a muon as final state particles; two reconstructed tracks, one of them from a muon and the second from a proton. Figure 4.8 shows one simulated CCQE interaction in the MINER $\nu$ A detector where a muon and a proton are visible. However, for a considerable fraction of interactions, the track (and kinematics) of the proton cannot be reconstructed because it is typically below detection threshold ( $\sim 200 \mathrm{MeV}$ kinetic energy). One of these events is shown in figure 4.9. Notice that the kinetic energy of the final state proton is highly correlated to the 4-momentum trasfer $Q^{2}$ of the interaction; the higher the $Q^{2}$ the more energy avaliable for the ejected proton which 
can be seen by comparing the $Q^{2}$ values in figures 4.8 and 4.9. On the other hand, nonCCQE interactions need to be excluded from the selected sample. One example of a common non-CCQE interaction was already shown in figure 4.7 .

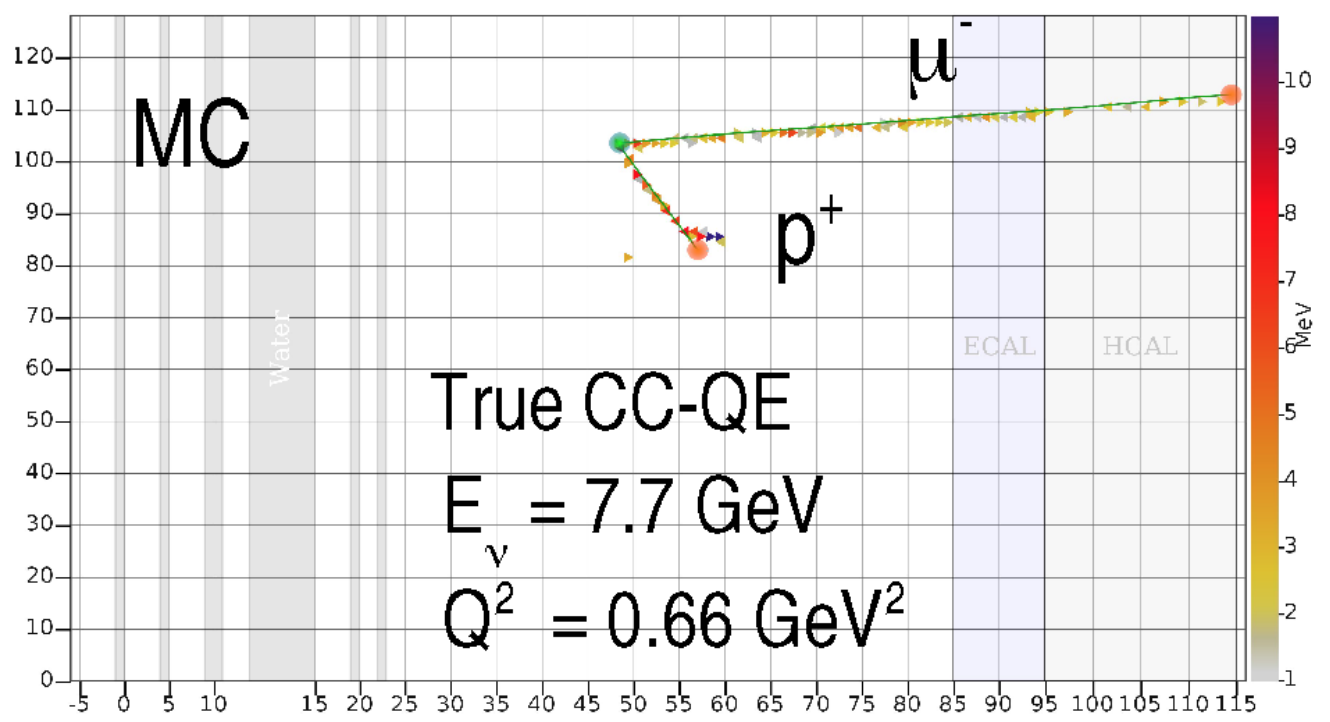

Figure 4.8: The MINER $\nu \mathrm{A}$ event display showing one view (the $\mathrm{X}$ view) of a simulated CCQE interaction where both, the final state muon and proton are visible.

In our analysis the identification of CCQE interactions relies basically on the detection of the muon and zero to low reconstructed recoil energy outside the vertex region. This way, CCQE interactions with proton kinetic energy below detection threshold can be included in the measurement. In addition, it allows us to include interactions in different nuclear configurations (such as two-nucleon correlations) that can have two or more nucleons in the final state and are, in the strict sense, not CCQE but are of interest to this work because they are a possible explanation to the excess of events seen by MiniBooNE as explained in section 2.5.1.

A charged-current quasi-elastic rich sample of events is selected, in data and MC, by applying the set of requirements described in the following sections, from 4.4.1 to 4.4.5.

\subsubsection{Muon Identification}

We select events with one reconstructed $\mu^{-}$. This requirement ensures that the muon started at MINER $\nu \mathrm{A}$ and has been completely reconstructed by range or curvature in the MINOS near detector. Muons that do not reach MINOS, either because they exit at a high angle or stop in the MINER $\nu \mathrm{A}$ detector, can not have the charge reconstructed and are not used in this 


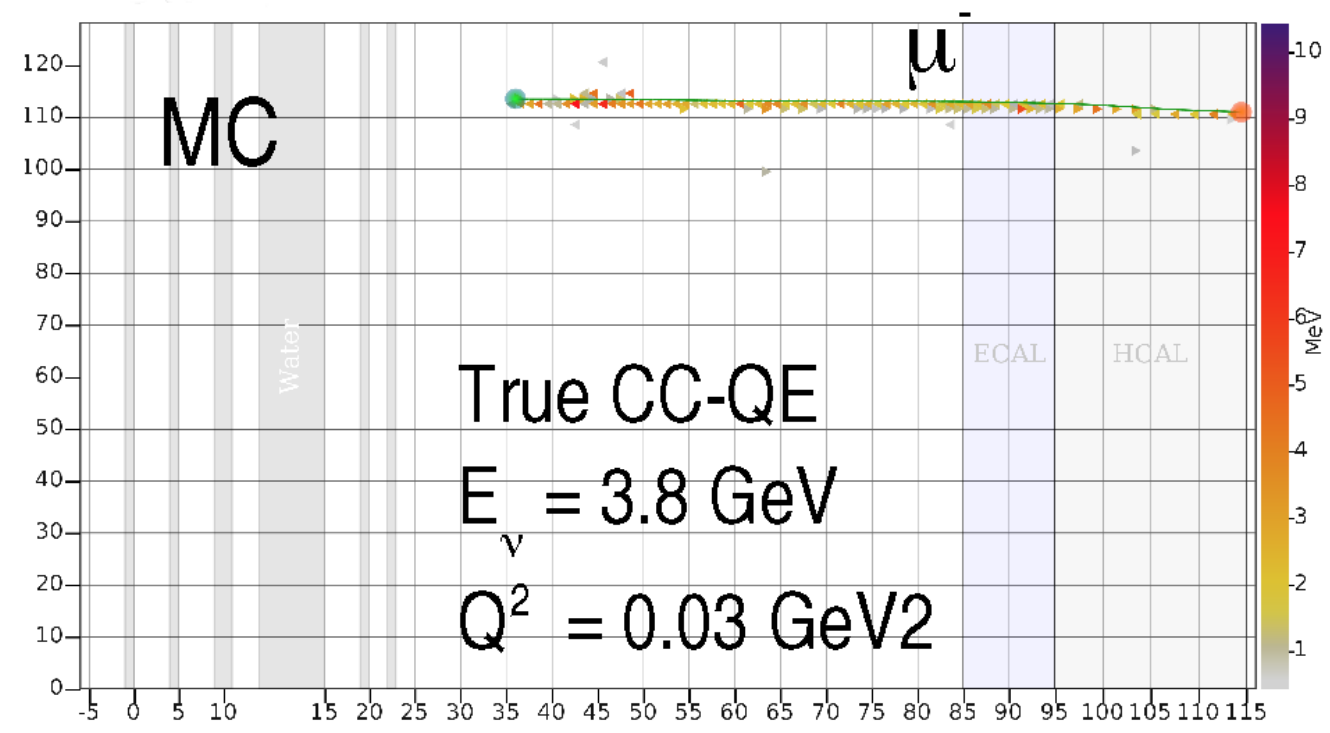

Figure 4.9: The MINER $\nu \mathrm{A}$ event display showing one view (the $\mathrm{X}$ view) of a simulated CCQE interaction where the final state proton is below detection threshold and cannot be detected.

analysis. Due to the relative position of the MINOS and MINER $\nu$ A detectors this requirement has an impact on the acceptance of events since only muons with energy above $\sim 2 \mathrm{GeV}$ and $\theta_{\mu}$ below $\sim 30^{\circ}$ can be identified. This requirement should remove from the selected sample all charged current anti-neutrino interactions that produce a $\mu^{+}$(wrong sign events). However, a neglible amount of them can get into the selected sample due to mis-reconstruction of the muon's charge.

\subsubsection{Fiducial Volume}

As mentioned in 4.3.1 the interaction vertex is determined by the beginning of the muon track. The interaction vertex is required to be inside a fiducial volume inside MINER $\nu$ A's active tracker region (plastic scintillator) whose limits are defined by hexagons of $850 \mathrm{~mm}$ apothem drawn in each module between module $27(z=5.980 \mathrm{~mm})$ and $80(z=8.422 \mathrm{~mm})$. The mass of the fiducial volume is 6.6 tons and it was estimated that it contains $1.51596 \times 10^{30}$ neutrons.

\subsubsection{Reconstructed Neutrino Energy}

Events have to have a reconstructed neutrino energy between $1.5 \mathrm{GeV}$ and $10 \mathrm{GeV}$ to be included in the selected sample. Events above $10 \mathrm{GeV}$, far away from the neutrino flux peak at $\sim 3 \mathrm{GeV}$, are rejected since they are mostly background (DIS) and are not used in the 
measurement. Due to the requirement of muon reconstruction in the MINOS detector, the sample does not contain events with less than $\sim 1.5 \mathrm{GeV}$ so they are removed explicitly.

\subsubsection{Number of Isolated Blobs}

We require that the event has less than three isolated blobs unattached to the vertex but in the fiducial volume. This requirement removes events with energetic showers. Figure 4.10 shows the distributions of isolated blobs for events with a muon in MINOS that starts in the fiducial volume. According to the MC, most of the events removed are deep inelastic scattering (DIS) and resonace (RES) that are likely to develop big showers. On the other hand, quasi-elastic events (QE) tend to have zero, one or two isolated blobs: zero isolated blobs is the case when the final state proton is completely contained in the vertex region (300 $\mathrm{mm}$ sphere around the vertex) and no energy outside it; one isolated blob can be formed when a proton exits the vertex region with enough energy to form an isolated blob. Much less frequently, two isolated blobs are formed when the isolated blob in the previous case is broken in two due to mis-reconstruction (missing clusters) or an actual reinteraction of the proton that produced neutral particles that decayed or interacted in a different part of the detector. We include the two isolated blobs case to be conservative and with the hope that non-QE events in this category can be removed later.

\subsubsection{Recoil Energy}

To avoid biasing the analysis by relying in the MC simulation of vertex energy (that is not complete), we select QE events by looking at the non-vertex recoil energy. The non-vertex recoil energy distribution of interactions passing the number of blobs cut is shown in figure 4.11 Vertex energy for neutrino and anti-neutrino interactions was studied very carefully and were published in [76] and, together with the present analysis, in [77].

The goal is to remove both DIS and RES events which tend to deposit larger amounts of energy outside the vertex region compared to QE events. To prevent the loss of QE events with high $Q^{2}$, that will also tend to deposit large amounts of energy, the value of the nonvertex recoil energy below which we select events will depend on $Q_{\mathrm{QE}}^{2}$. For this purpose, the $Q_{\mathrm{QE}}^{2}$ variable is binned in the following way ${ }^{2}:[0.0,0.025,0.05,0.1,0.2,0.4,0.8,1.2,2.0]$. We then analyse the non-vertex recoil energy distributions in each bin of $Q_{\mathrm{QE}}^{2}$ to select an appropiate cut value. Figure 4.12 shows the non-vertex recoil energy distribution for each of the eight $Q_{\mathrm{QE}}^{2}$ bins considered.

For each bin in $Q_{\mathrm{QE}}^{2}$, we calculate an efficiency and purity of events with non-vertex recoil energy lower than a given value, $E_{c u t}$, using the Monte Carlo simulation and according to the

\footnotetext{
${ }^{2}$ The size of the bins are related to the resolution of the MINER $\nu \mathrm{A}$ detector.
} 


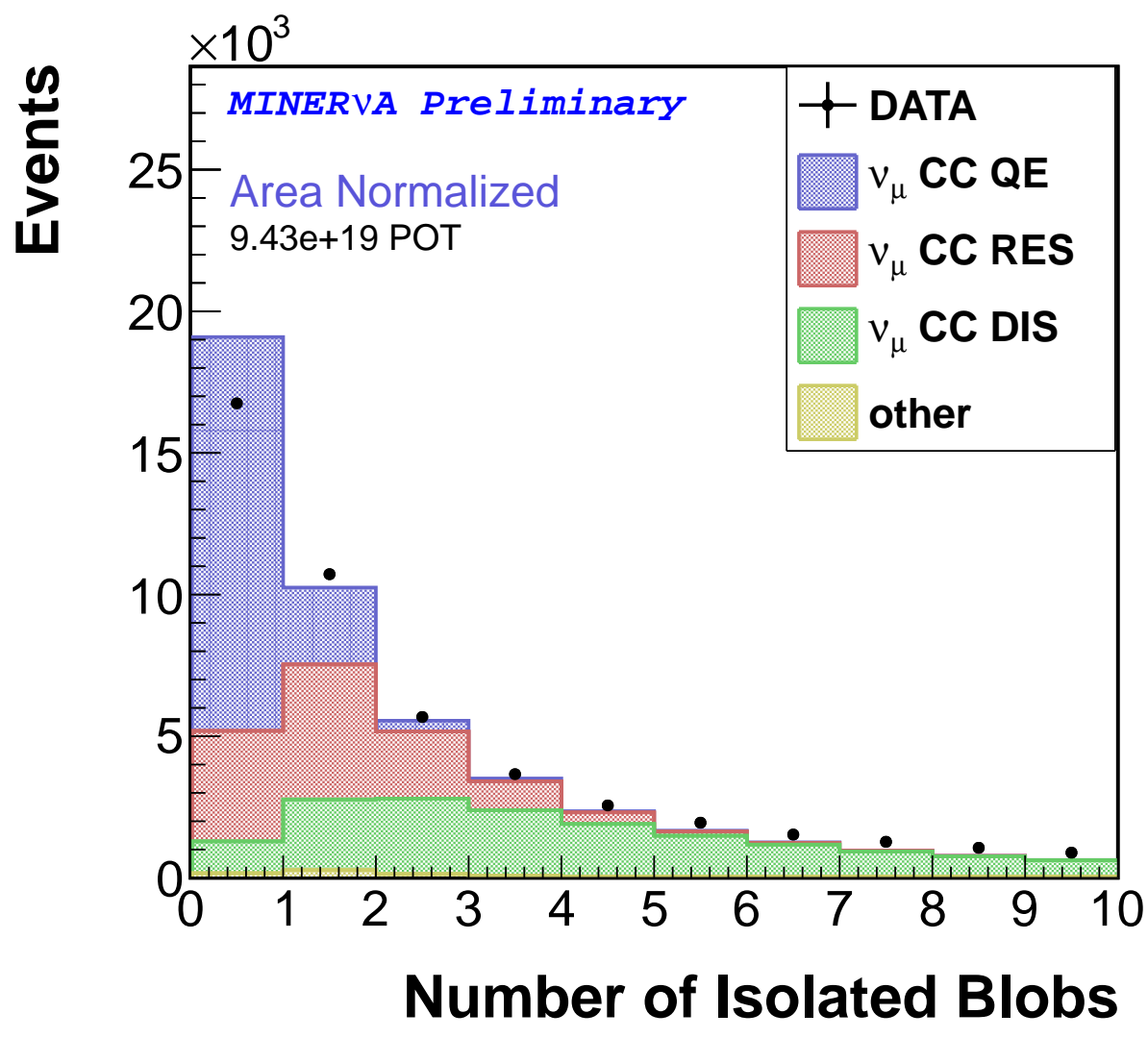

Figure 4.10: The number of isolated blobs found away from the vertex but in the fiducial volume in data and the MC simulation.

following formulas,

where:

$$
\begin{gathered}
\operatorname{efficiency}_{i}\left(E_{\text {cut }}\right)=\frac{N_{C C Q E}^{i, \text { selected }}\left(E_{\text {cut }}\right)}{N_{C C Q E}^{i, t o t a l}}, \\
\operatorname{purity}_{i}\left(E_{\text {cut }}\right)=\frac{N_{C C Q E}^{i, \text { selected }}\left(E_{\text {cut }}\right)}{N_{\text {All }}^{i, \text { selected }}\left(E_{\text {cut }}\right)}
\end{gathered}
$$

- $i$ is the $Q_{\mathrm{QE}}^{2}$ bin being analyzed.

- $N_{C C Q E}^{i, \text { selected }}\left(E_{\text {cut }}\right)$ is the number of CCQE events with non-vertex recoil energy lower than $E_{\text {cut }}$.

- $N_{C C Q E}^{i, t o t a l}$ is the number of events before the non-vertex recoil energy requirement.

- $N_{\text {All }}^{i \text { selected }}\left(E_{\text {cut }}\right)$ is the total number of events with non-vertex recoil energy lower than $E_{\text {cut }}$.

These efficiencies and purities are shown in Figure 4.13 together with their product ef ficiency $\times$ purity. We choose the minimum non-vertex recoil energy cut where the efficiency is equal or 


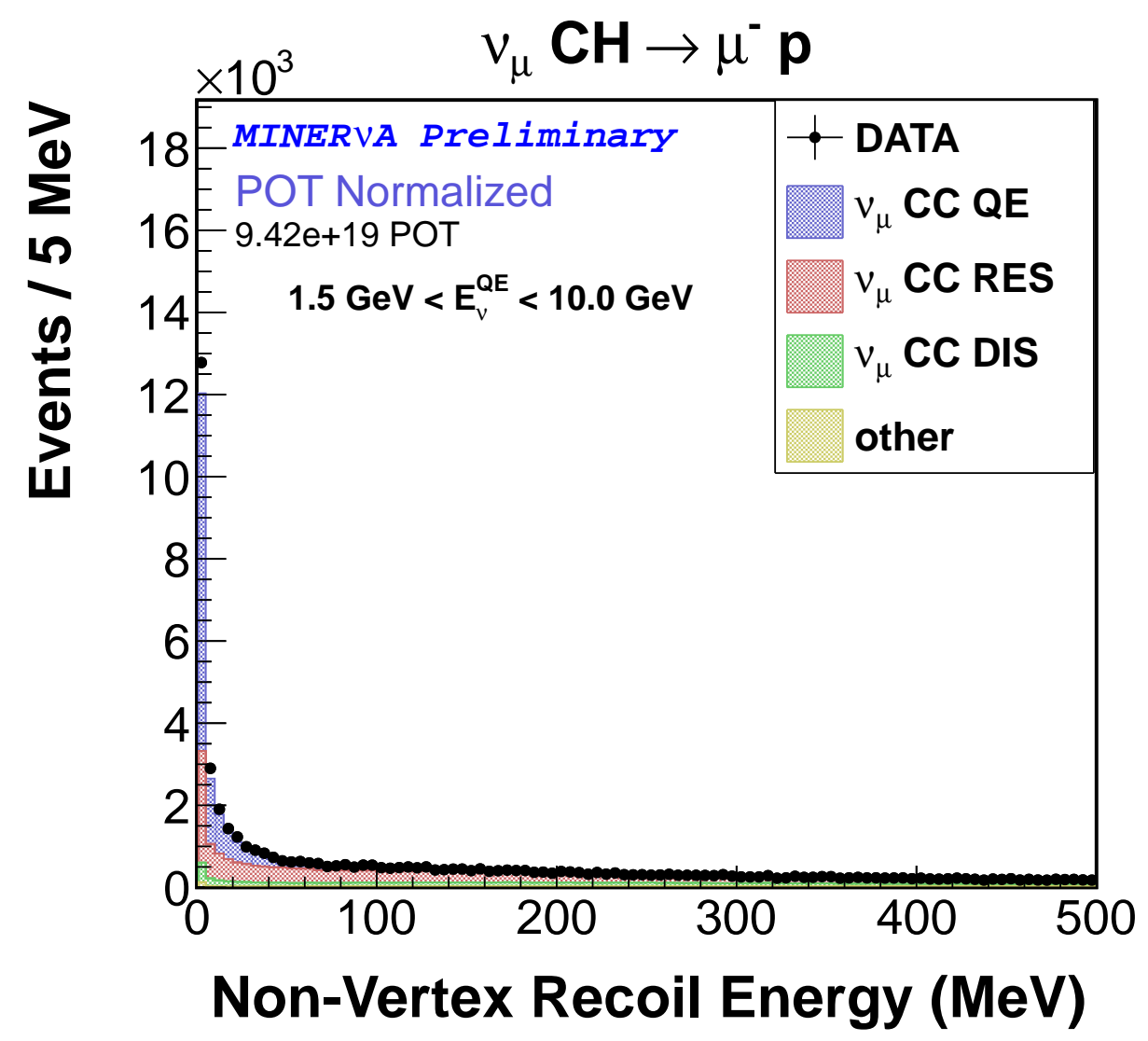

Figure 4.11: Non-vertex recoil energy distributions of CC interactions in the fiducial volume and with less than three isolated blobs.

greater than $95 \%$ instead of the "optimal cut" where efficiency $\times$ purity is maximum. The choosen cut is a conservative one that selects $\sim 95 \%$ of the CCQE events but, at the same time, accepts non-CCQE events that could be filtered using a tighter cut closer to the "optimal cut" value whitout losing too much CCQE events. We don't use the "optimal cut" value to improve purity because we don't want to reject signal events, such as multi-nucleon final states due to interactions with correlated nucleons in the nucleus (multi-nucleon mechanisms), from the data that won't be possible to recover later using corrections based on a Monte Carlo simulation that do not include this kind of events. The choosen cut is shown as a red vertical line in figure 4.13

After following this procedure, the result is a discrete $Q_{\mathrm{QE}}^{2}$-dependent non-vertex recoil energy cut. In order to have a continuos cut, we use the function $\operatorname{SignalCut}\left(Q_{\mathrm{QE}}^{2}\right)$ in equation 4.9 , which is the result of a fit to a quadratic function of the discrete values of the $Q_{\mathrm{QE}^{-}}^{2}$ dependent non-vertex recoil energy cut. At high values of $Q_{\mathrm{QE}}^{2}(g e q 1.61 \mathrm{GeV})$, a maximum non-vertex recoil energy cut is used to avoid too high values due to the quadratic function. At low values of $Q_{\mathrm{QE}}^{2}(<0.166 \mathrm{GeV})$, a minimum non-vertex recoil energy cut is used to avoid 

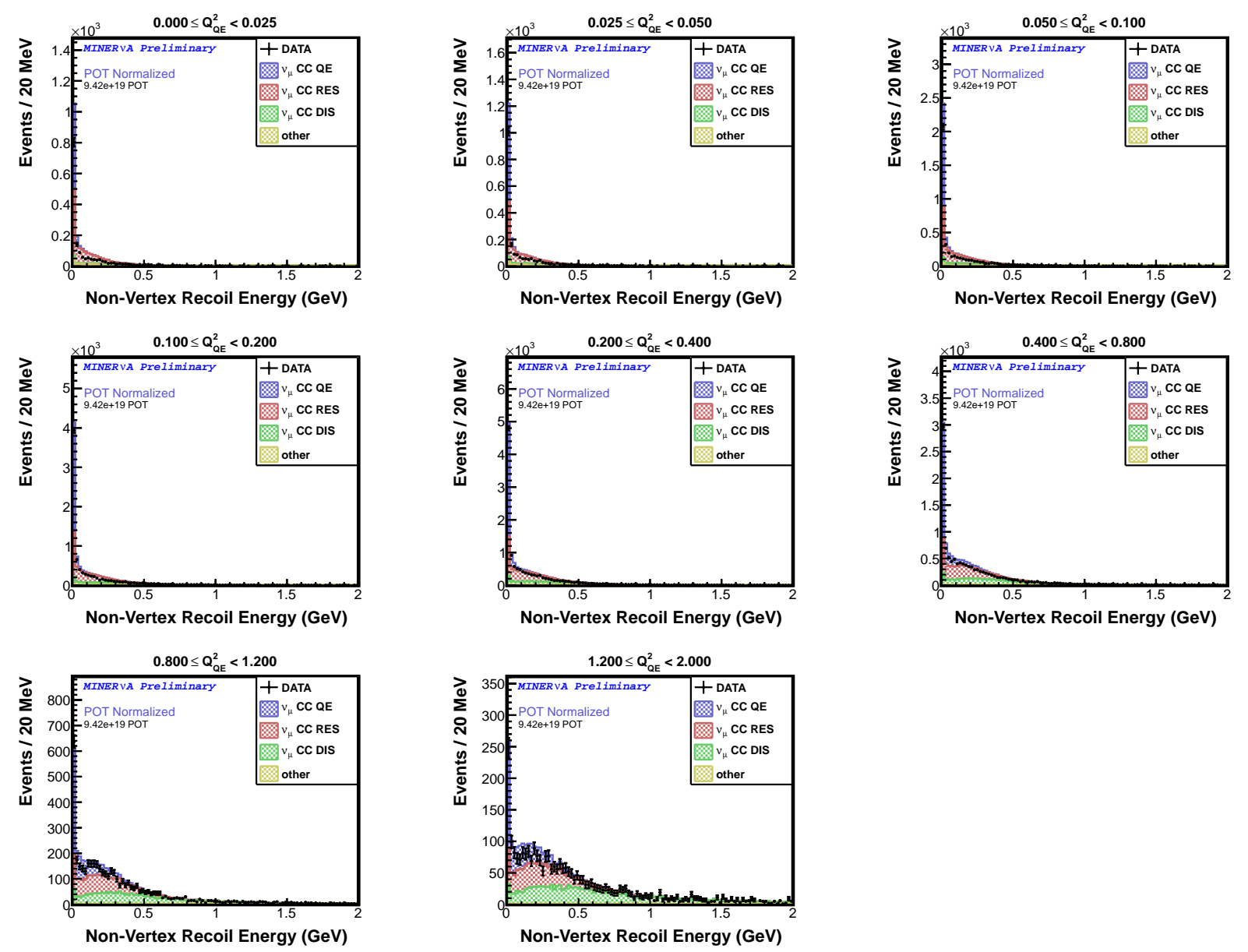

Figure 4.12: Non-vertex recoil energy distributions of CC interactions in the fiducial volume and with less than three isolated blobs in bins of $Q_{\mathrm{QE}}^{2}$.

values very close to zero due to the quadratic function. All $Q_{\mathrm{QE}}^{2}$ values and $\operatorname{SignalCut}\left(Q_{\mathrm{QE}}^{2}\right)$ are expresed in $\mathrm{GeV}$.

$$
\text { SignalCut }\left(Q_{\mathrm{QE}}^{2}\right)= \begin{cases}0.05 & \text { if } Q_{\mathrm{QE}}^{2}<0.166 \\ -0.05+0.64 Q_{\mathrm{QE}}^{2}-0.22\left(Q_{\mathrm{QE}}^{2}\right)^{2} & \text { if } 0.166 \leq Q_{\mathrm{QE}}^{2}<1.61 \\ 0.41 & \text { if } Q_{\mathrm{QE}}^{2} \geq 1.61\end{cases}
$$

An additional function, placed at $0.5 \mathrm{GeV}$ above $\operatorname{SignalCut}\left(Q_{\mathrm{QE}}^{2}\right)$, is also constructed and called SidebandCut $\left(Q_{\mathrm{QE}}^{2}\right)$. The formula of SidebandCut $\left(Q_{\mathrm{QE}}^{2}\right)$ is presented in equation 4.10.

$$
\text { SidebanCut }\left(Q_{\mathrm{QE}}^{2}\right)= \begin{cases}0.55 & \text { if } Q_{\mathrm{QE}}^{2}<0.166 \\ 0.45+0.64 Q_{\mathrm{QE}}^{2}-0.22\left(Q_{\mathrm{QE}}^{2}\right)^{2} & \text { if } 0.166 \leq Q_{\mathrm{QE}}^{2}<1.61 \\ 0.91 & \text { if } Q_{\mathrm{QE}}^{2} \geq 1.61\end{cases}
$$



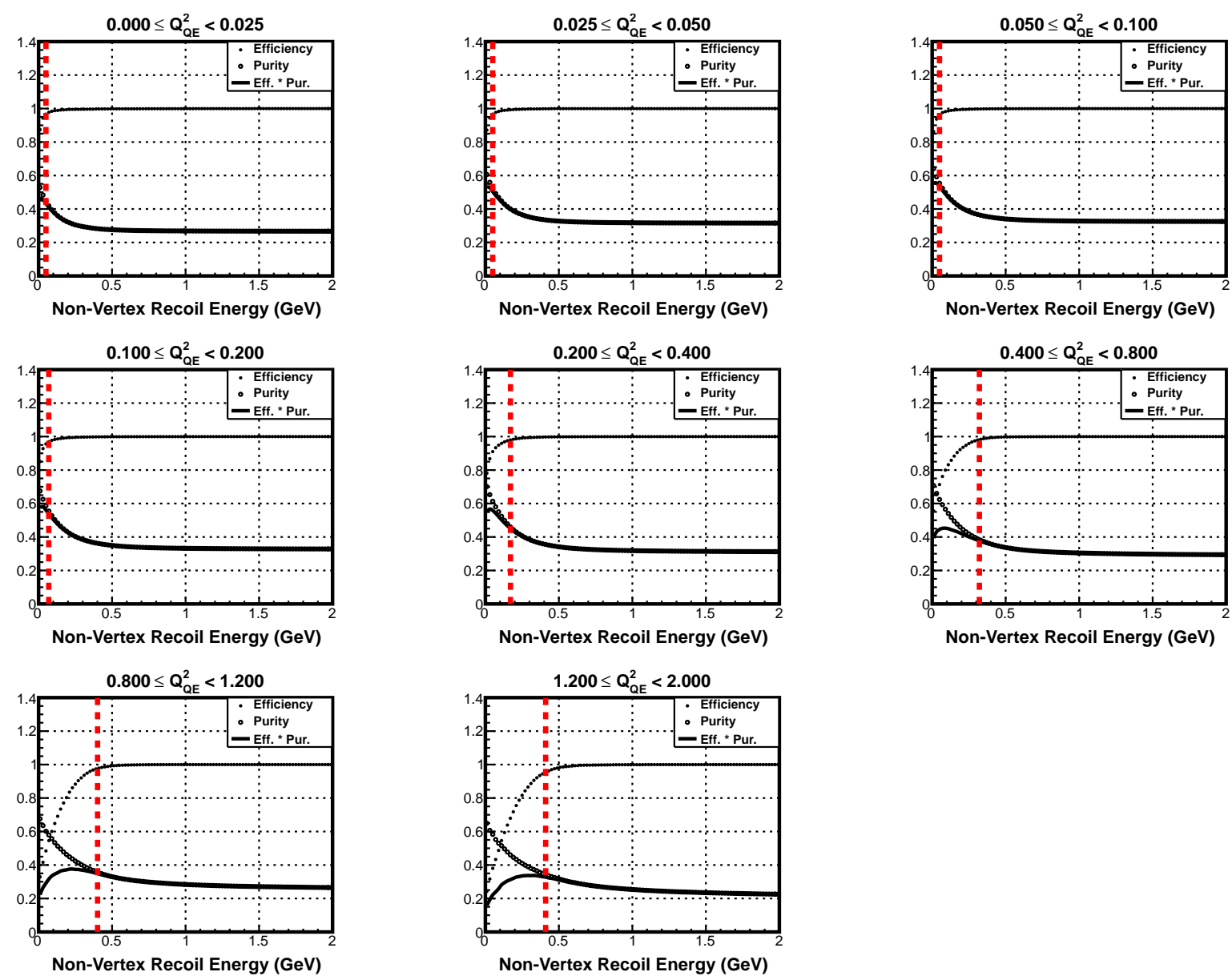

Figure 4.13: Efficiency, purity and their product as a function of a potential non-vertex recoil energy cut for each bin of $Q_{\mathrm{QE}}^{2}$ considered in the analysis. The vertical red line in each plots is the selected cut that correspond to the minimum cut where the efficiency is $95 \%$ or bigger.

Figure 4.14 shows the distribution of simulated events as a function of non-vertex recoil energy and $Q_{\mathrm{QE}}^{2}$ together with the $\operatorname{SignalCut}\left(Q_{\mathrm{QE}}^{2}\right)$ and $\operatorname{SidebandCut}\left(Q_{\mathrm{QE}}^{2}\right)$ curves. Events falling below the SignalCut $\left(Q_{\mathrm{QE}}^{2}\right)$ curve populates our selected sample of CCQE candidates that we will call signal sample. Events falling between $\operatorname{SignalCut}\left(Q_{\mathrm{QE}}^{2}\right)$ and $\operatorname{SidebandCut}\left(Q_{\mathrm{QE}}^{2}\right)$ define the sideband sample. Events in the sideband sample are events with very similar characteristic than to the ones in the signal sample but, because of the higher recoil energy, they will contain a big fraction of non-CCQE background events. Due to this, we can use the sideband sample to constraint the non-CCQE background in the signal sample later in the analysis. 


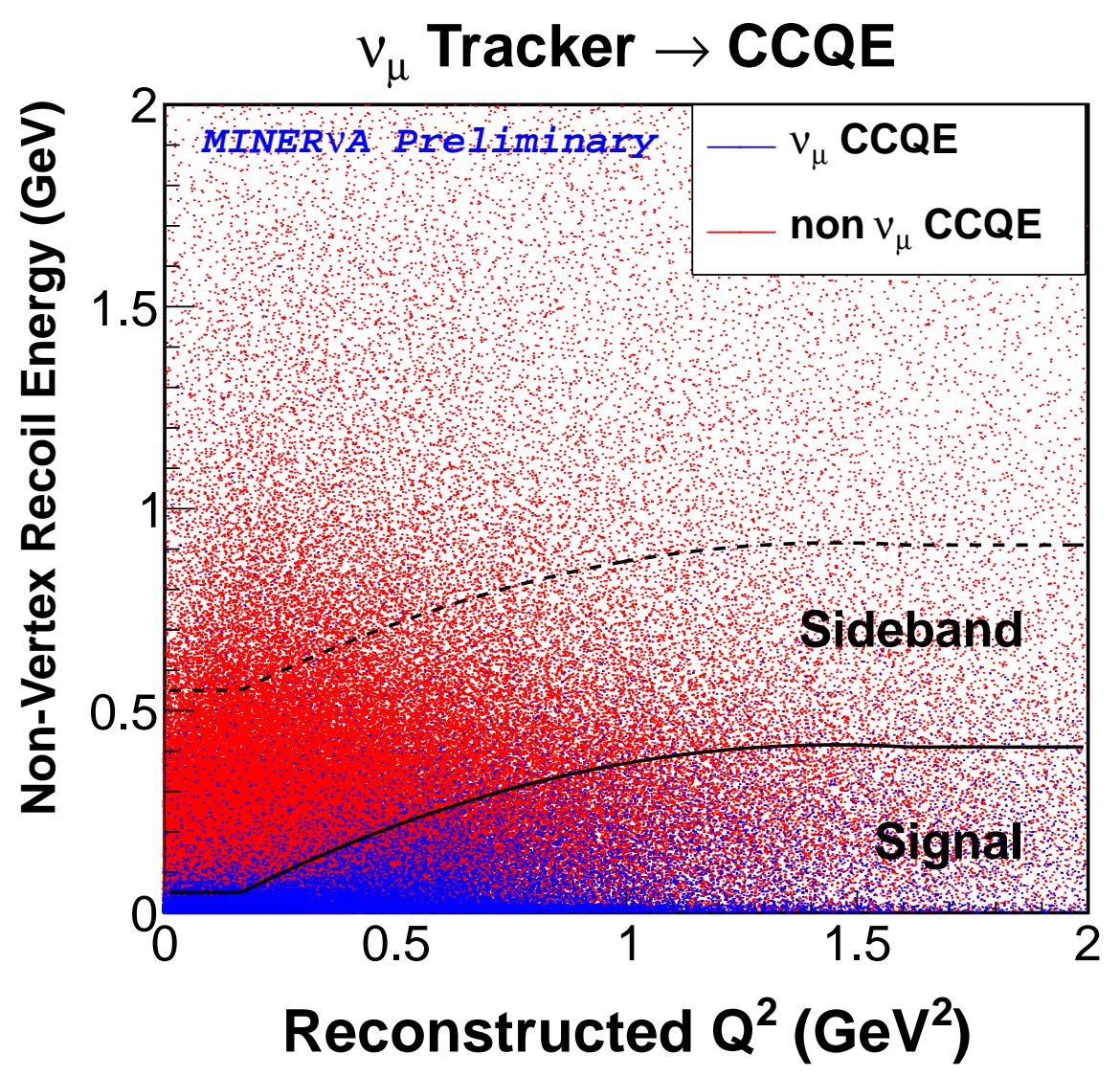

Figure 4.14: Non-vertex recoil energy versus the reconstructed $Q_{\mathrm{QE}}^{2}$ distribution of CC interactions in the fiducial volume and with less than three isolated blobs according to the Monte Carlo simulation. CCQE events are in red and non-CCQE events are in blue.

\subsection{Selected Sample}

After apply the event selection to data, 29,620 events were selected as CCQE interactions candidates. Figure 4.15 shows the distribution of selected events (signal region in Figure 4.14) as a function of reconstructed $E_{\nu}^{\mathrm{QE}}$ and $Q_{\mathrm{QE}}^{2}$. A discrepancy between the MC simulation and data can be observed, which is biggest at low values of $Q_{\mathrm{QE}}^{2}$. The source of the discrepancy can be due to a bad model of the signal (CCQE) or the background (non-CCQE). In order to avoid the ambiguity, we will constraint the non-CCQE background with data later in the analysis. Distributions of the selected sample in additional reconstructed variables can be found in $\mathrm{A}$

According to the MC simulation, the total CCQE selection efficiency is $47 \%$ and the sample purity, $49 \%$. They were calculated using the following equations,

$$
\text { efficiency }=\frac{N_{C C Q E}^{\text {Selected }}}{N_{C C Q E}^{\text {Total }}}
$$



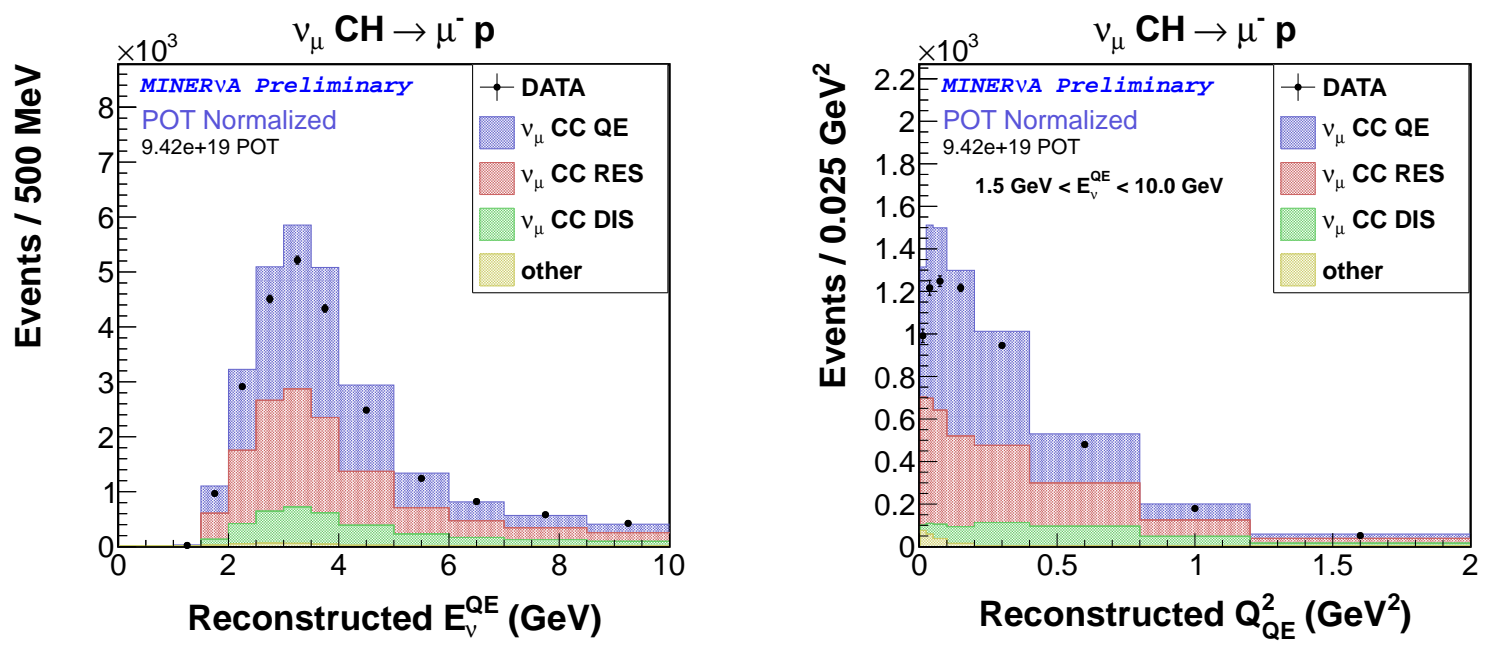

Figure 4.15: Distribution of CCQE event candidates as a function of $E_{\nu}^{\mathrm{QE}}$ and $Q_{\mathrm{QE}}^{2}$. The $\mathrm{MC}$ sample have been scaled to match the data POT (POT normalization).

$$
\text { purity }=\frac{N_{C C Q E}^{\text {Selected }}}{N_{\text {All }}^{\text {Selected }}}
$$

Where:

- $N_{C C Q E}^{\text {Selected }}$ is the number of MC signal events (CCQE interactions) in the selected sample.

- $N_{C C Q E}^{\text {Total }}$ is the total number of MC signal events generated.

- $N_{\text {All }}^{\text {Selected }}$ is the number of events in the selected sample.

These quantities can be seen as a function of the generated $Q_{\mathrm{QE}}^{2}$ and $E_{\nu}^{\mathrm{QE}}$ in Figure 4.16 and 4.17. Generated $Q_{\mathrm{QE}}^{2}$ and $E_{\nu}^{\mathrm{QE}}$ are defined by formulas 4.6 and 4.5 using generated (true) instead of the reconstructed muon kinematics. This is consistent with our interpretion of $Q_{\mathrm{QE}}^{2}$ and $E_{\nu}^{\mathrm{QE}}$ not as the $Q^{2}$ and $E_{\nu}$ of the interaction but as a parametrization of the muon kinematics.

Efficiencies and purities as a function of additional variables can be found in appendix A. 

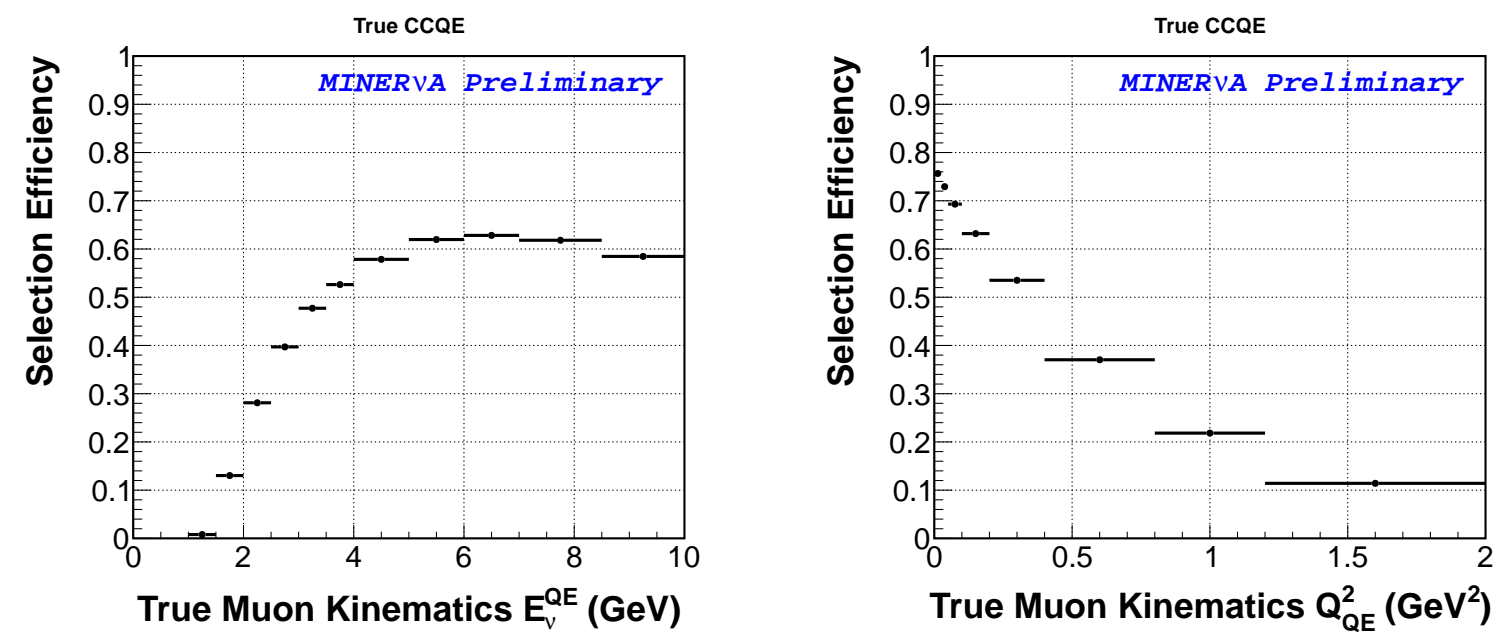

Figure 4.16: Selection efficiency as a function of generated $E_{\nu}^{\mathrm{QE}}$ (left) and $Q_{\mathrm{QE}}^{2}$ (right).
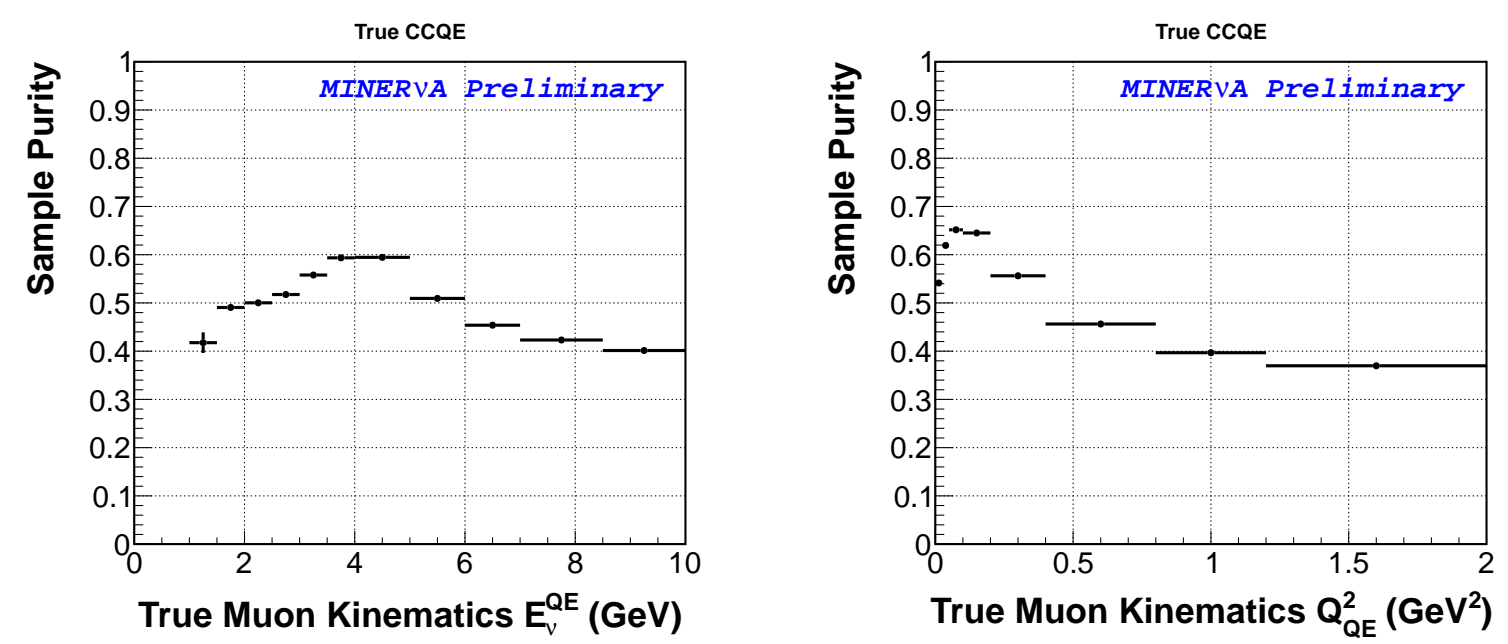

Figure 4.17: Selected sample purity as a function of generated $E_{\nu}^{\mathrm{QE}}$ (left) and $Q_{\mathrm{QE}}^{2}$ (right). 


\section{Chapter 5}

\section{Measuring the Differential Cross section $d \sigma / d Q_{Q E}^{2}$}

\subsection{Introduction}

The goal of this section is to describe the measurement of the $\nu_{\mu}$ differential cross section of charged current quasi-elastic (CCQE) interactions on plastic scintillator in the MINER $\nu \mathrm{A}$ detector.

In order to calculate the cross section from the number of reconstructed events identified as CCQE candidates in the previous chapter 4, we correct the distribution of CCQE candidates for expected background events rates, known kinematic smearing effects in the reconstruction and selection efficiency. The corrected distribution is then normalized by the predicted integral of the flux and number of targets in the fiducial volume. All this is summarized by equation 5.1 that give us the quasi-elastic diferential cross section in the $i^{\text {th }}$ bin of $Q_{\mathrm{QE}}^{2}$ :

$$
\left(\frac{d \sigma}{d Q_{\mathrm{QE}}^{2}}\right)_{i}=\frac{1}{\Phi_{\nu} \times T_{n}} \cdot \frac{1}{\left(\Delta Q_{\mathrm{QE}}^{2}\right)_{i}} \cdot \frac{\sum_{j} U_{i j}\left[N_{j}^{\text {data }}-N_{j}^{b g}\right]}{\varepsilon_{i}}
$$

where:

- $\Phi_{\nu}=\int \phi\left(E_{\nu}\right) d E_{\nu} \quad$ is the total neutrino flux over the region which contributes to the event sample $(1.5 \mathrm{GeV}$ to $10 \mathrm{GeV})$.

- $T_{n}$ is the total number of neutrons in the fiducial volume.

- $\left(\Delta Q_{\mathrm{QE}}^{2}\right)_{i}$ is the width of bin $i$. The bins are the same used for the recoil energy as in section 4.4.5: $[0.0,0.025,0.05,0.1,0.2,0.4,0.8,1.2,2.0]$. 
- $N_{j}^{\text {data }}$ is the measured distribution of selected (CCQE) events in bins of reconstructed $Q_{\mathrm{QE}}^{2}$.

- $N_{j}^{b g}$ is an estimate for the number of background (non-CCQE) events in bins of reconstructed $Q_{\mathrm{QE}}^{2}$.

- $U_{i j}$ is a matrix that describes the migration from the true $Q_{\mathrm{QE}}^{2}$ bin $i$ to the reconstructed $Q_{\mathrm{QE}}^{2}$ bin $i$, due to finite resolutions and realistic biases of the reconstruction.

- $\varepsilon_{i}$ is the efficiency for reconstructing and selecting signal events as a function of the true variable.

We rely heavily on the simulation to obtain these corrections that are certainly model dependent; therefore, it is important to check the MC accuracy and to adequately assess sources of systematic uncertainty.

\subsubsection{Background Constraint and Subtraction}

The MC simulation (based on the GENIE neutrino event generator) allows us to predict the level of non-CCQE backgrounds that can not be suppressed by the CCQE event selection described in section 4.4 and the result shown in 4.15 where a discrepancy between MC and data is found. This background consists mainly of resonant pion production (RES) and deep inelastic scattering (DIS) interactions as can be seen in the distribution of CCQE event cadidates shown in figure 4.15. They enter the signal sample because the recoil final state particles (mostly pions) are contained in the sphere of $300 \mathrm{~mm}$ around the interaction vertex or are absorbed before exiting the atomic nucleus. The rate of RES interactions is simulated by the Rein-Seghal model and it is known to have big uncertainties. To avoid a big dependece on the model, we use MINER $\nu$ A data to constraint the non-CCQE background prediction in the selected sample of CCQE candidates.

In order to constraint the non-CCQE background prediction, we use the sideband sample as defined in figure 4.14. The sideband sample is dominated by non-CCQE background events with a topology similar to the signal sample and can be used to study the non-CCQE background. Figure 5.1 shows the reconstructed $Q_{\mathrm{QE}}^{2}$ distribution of events in the sideband sample where one can notice the small amount of CCQE events.

From this distribution we compute a background scale as a function of reconstructed $Q_{\mathrm{QE}}^{2}$ which is a factor that if applied to background events in the sideband, data and MC agree perfectly. This can be achieved by defining the background scale as:

$$
B S_{i}=\frac{N_{i}^{\text {data }}-N_{i}^{C C Q E}}{N_{i}^{\text {non-CCQE }}}
$$




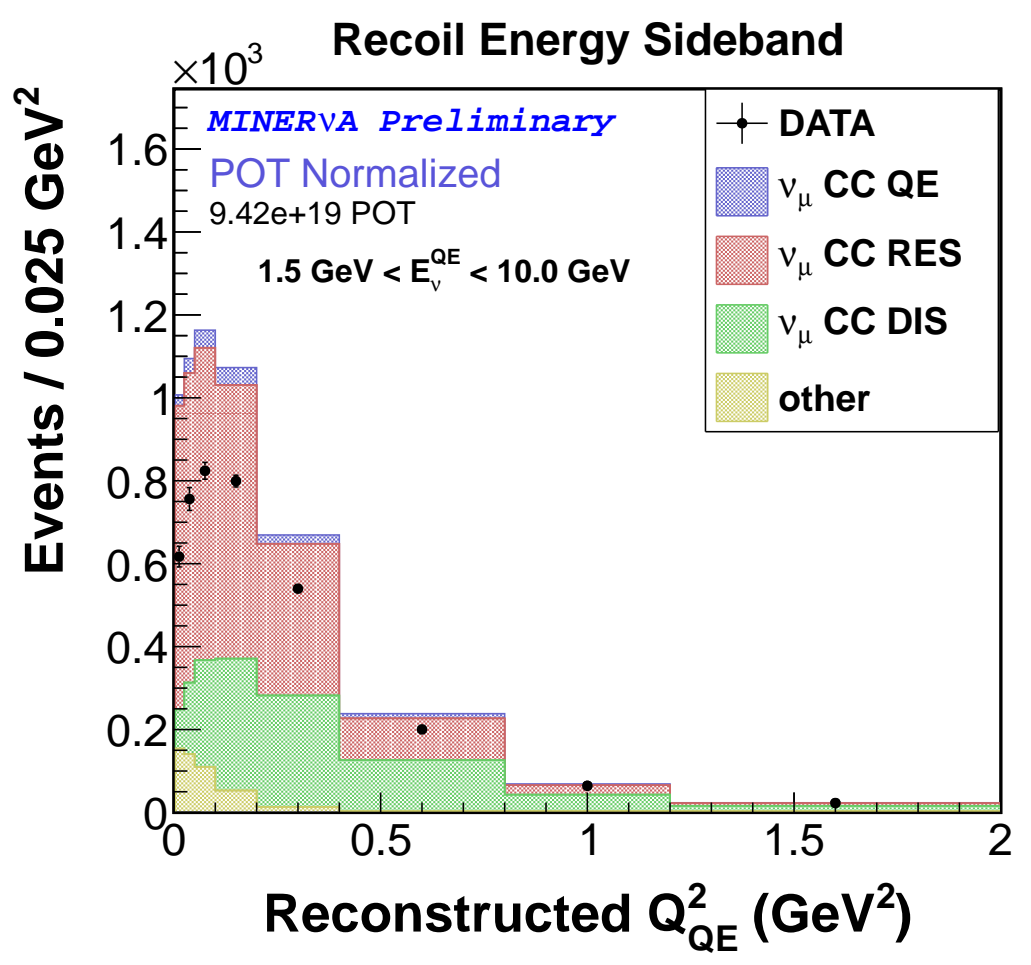

Figure 5.1: Reconstructed $Q_{\mathrm{QE}}^{2}$ distribution of events in the sideband sample.

Where:

- $i$ represents the $i^{\text {th }} Q_{\mathrm{QE}}^{2}$ bin.

- $B S_{i}$ is the background scale calculated for bin $i$.

- $N_{i}^{\text {data }}$ is the number of data events found in bin $i$.

- $N_{i}^{C C Q E}$ is the number of simulated CCQE events falling in bin $i$.

- $N_{i}^{\text {non-CCQE }}$ is the number of simulated non-CCQE (background) events falling in bin $i$.

Figure 5.2 shows the resulting background scale.

It can be seen that, in the sideband sample, the non-CCQE backgrounds are being overstimated as high as $50 \%$ in the low values of $Q_{\mathrm{QE}}^{2}$. Events in the sideband sample are close enough to the signal sample in terms of recoil energy (see Figure 4.14) so we can use the background scale to scale the non-CCQE background found in the signal region. We then subtract the constraint background from the event candidates distribution and the result is shown in Figure 5.3 . 


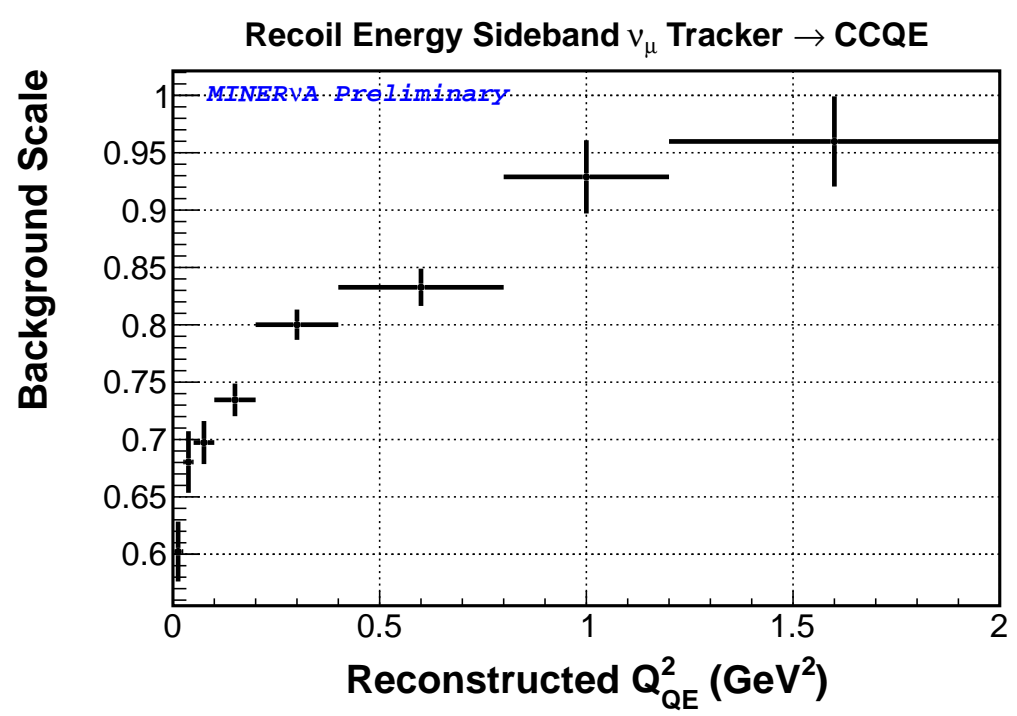

Figure 5.2: Background scale needed to the background in order to have perfect data-MC agreement in the reconstructed $Q_{\mathrm{QE}}^{2}$ distribution of events in the Sideband sample.
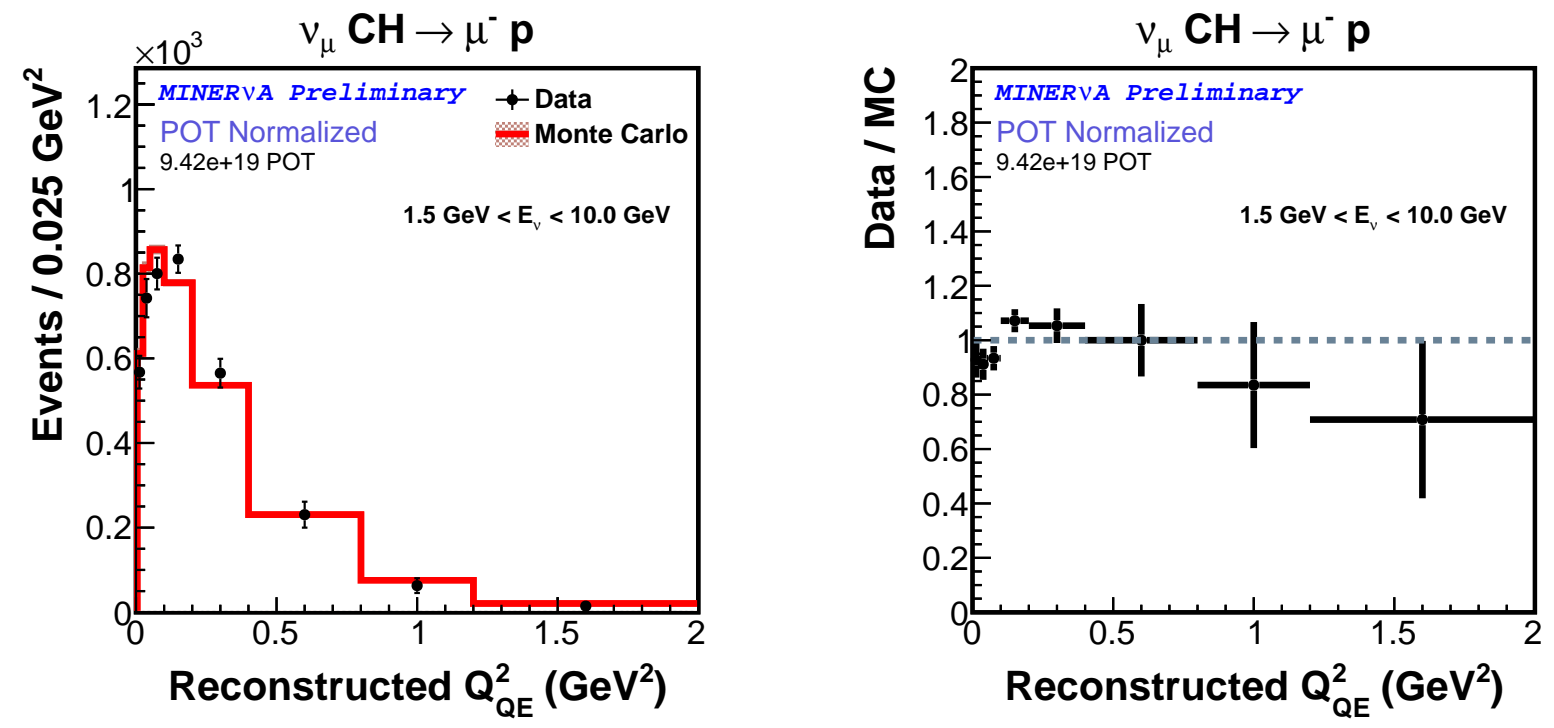

Figure 5.3: Background subtracted distribution of events in bins of reconstructed $Q_{\mathrm{QE}}^{2}$ as defined in equation 4.6 (left). The ratio between data and MC (right).

\subsubsection{Unfolding Detector Smearing}

After the background subtraction, we correct for bin migrations effects by using an unfolding technique. The general problem of unfolding consists in estimating a probability distribution where data is subject to random fluctuations due to limited resolution. Without unfolding, a measurement cannot be directly compared with the results of other experiments. In this analysis, the quantities measured by the detector are $E_{\mu}$ and $\theta_{\mu}$. Since $Q_{\mathrm{QE}}^{2}$ is a parametrization of the muon kinematics, the finite resolution in $E_{\mu}$ and $\theta_{\mu}$ generates a migration from a generated 
(true) value of $Q_{\mathrm{QE}}^{2}$ to the different reconstructed value. We use the MC simulation to construct a migration matrix that contains the probability of generated (true) values in a $Q_{\mathrm{QE}}^{2}$ bin to migrate to a different bin when its reconstructed value is considered. This matrix is especific for each experiment and depends on the design and properties of the detector. Figure 5.4 shows the migration matrix for the chosen $Q_{\mathrm{QE}}^{2}$ bins in the MINER $\nu \mathrm{A}$ detector.
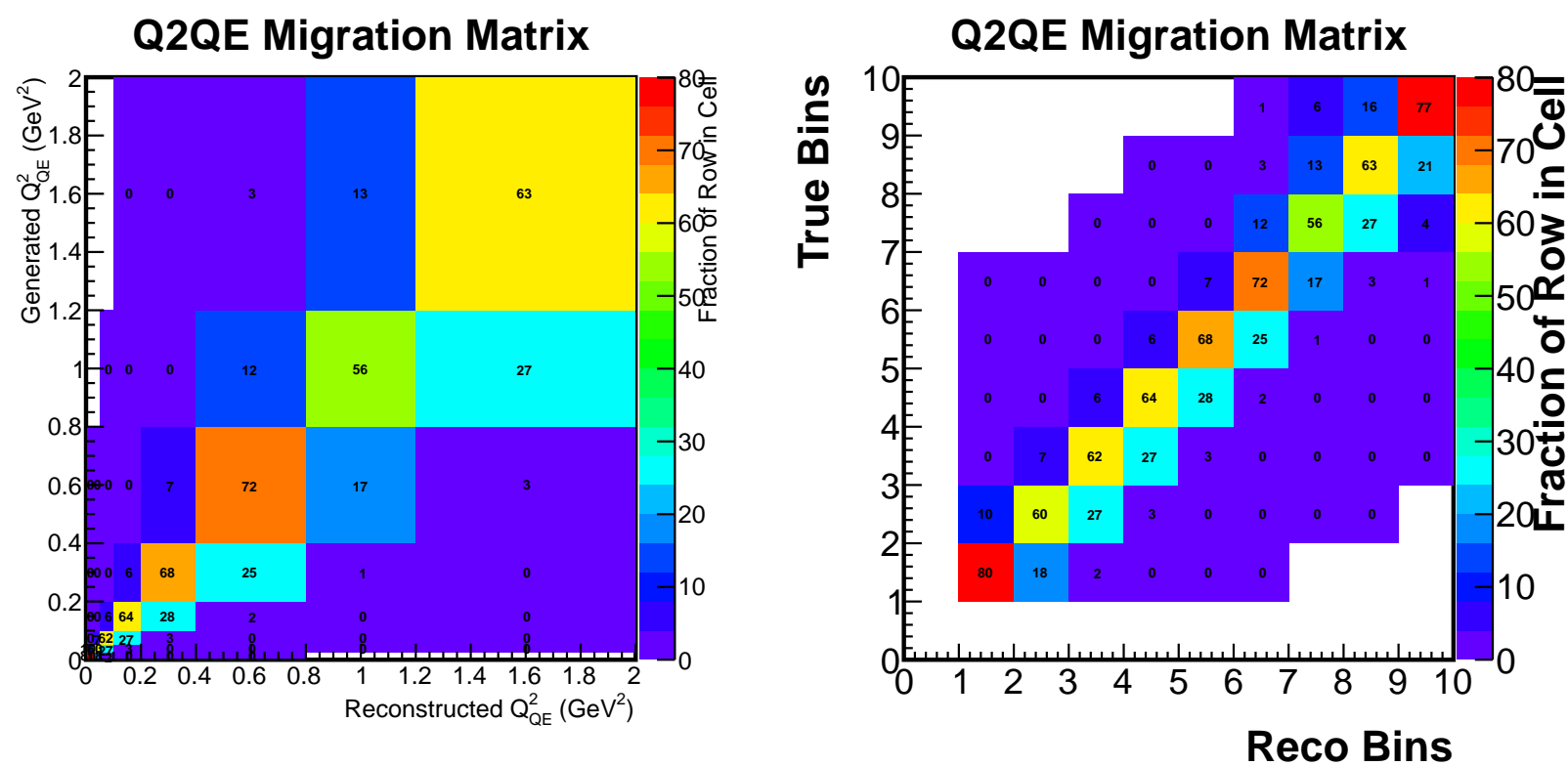

Figure 5.4: Migration matrix for the $Q_{\mathrm{QE}}^{2}$ bins in the MINER $\nu$ A detector. Right plot's axis shows the actual $Q_{\mathrm{QE}}^{2}$ bins in $\mathrm{GeV}^{2}$. Left plot's axis shows the number of bins. Notice that underflow and overflow bins are considered.

The unfolding performed is the Bayesian unfolding method [78] with four iterations. This technique allow us to estimate the true $Q_{\mathrm{QE}}^{2}$ distribution from the reconstructed $Q_{\mathrm{QE}}^{2}$ distribution and the migration matrix. The result is shown in Figure 5.5.

\subsubsection{Efficiency Correction}

After the unfolding, we obtained the true $Q_{\mathrm{QE}}^{2}$ distribution of detected signal events in the MINER $\nu \mathrm{A}$ detector. In order to calculate the total number of signal events, detected or not, we use an efficiency correction. The efficiency is defined by equation 4.11 and is a convolution of the MINER $\nu \mathrm{A}$ (and MINOS) detector acceptances, the muon tracking efficiency in both detectors, the muon track matching efficiency between MINER $\nu \mathrm{A}$ and MINOS, and the efficiency on selecting signal events.

The efficiency as a function of true $Q_{\mathrm{QE}}^{2}$ is shown in Figure 4.16. According to equation 5.1 , we correct the unfolded distribution by dividing by the efficiency . The result is shown in Figure 5.6. 

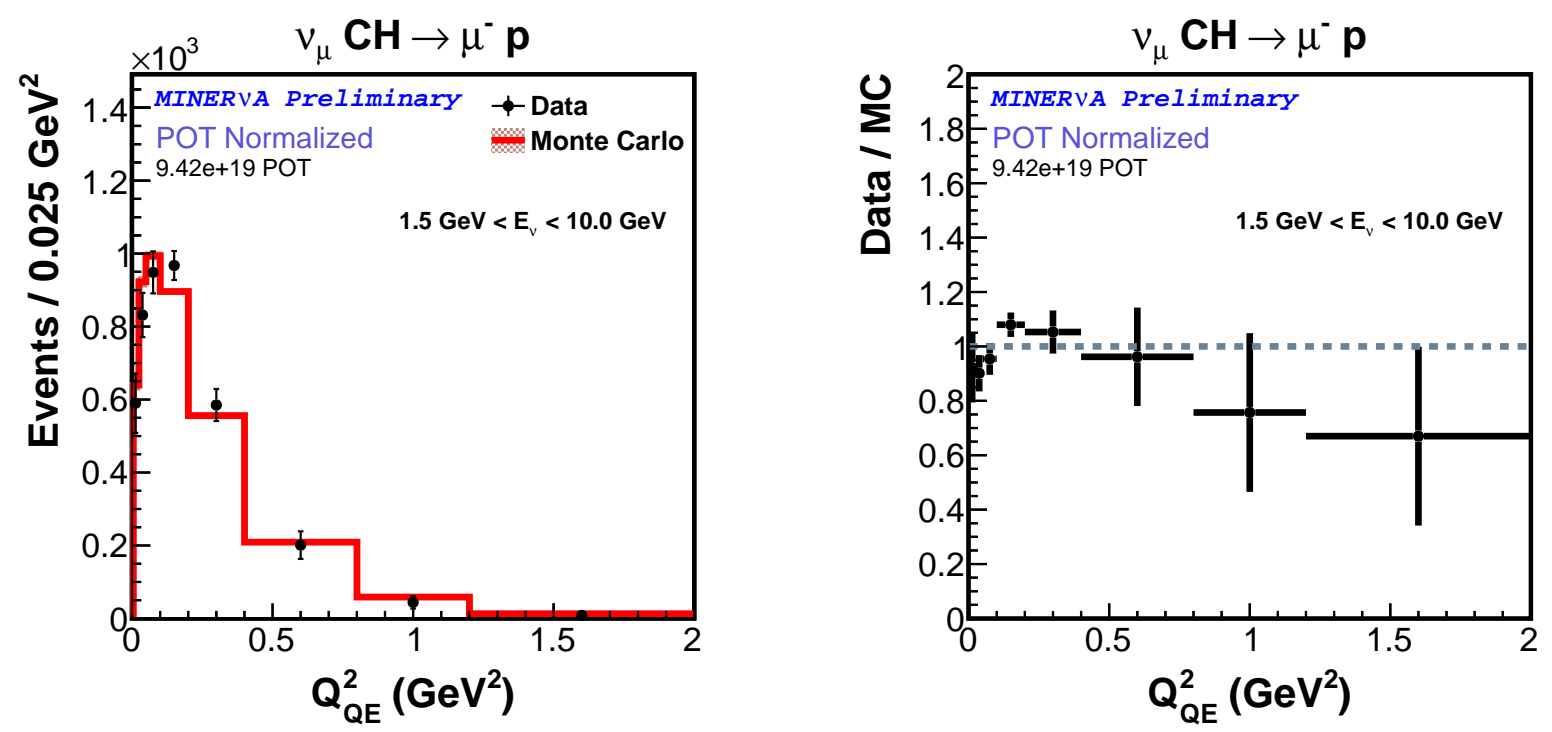

Figure 5.5: Background subtracted and unfolded distribution of events in bins of true $Q_{\mathrm{QE}}^{2}$ (left). The ratio between data and MC (right).
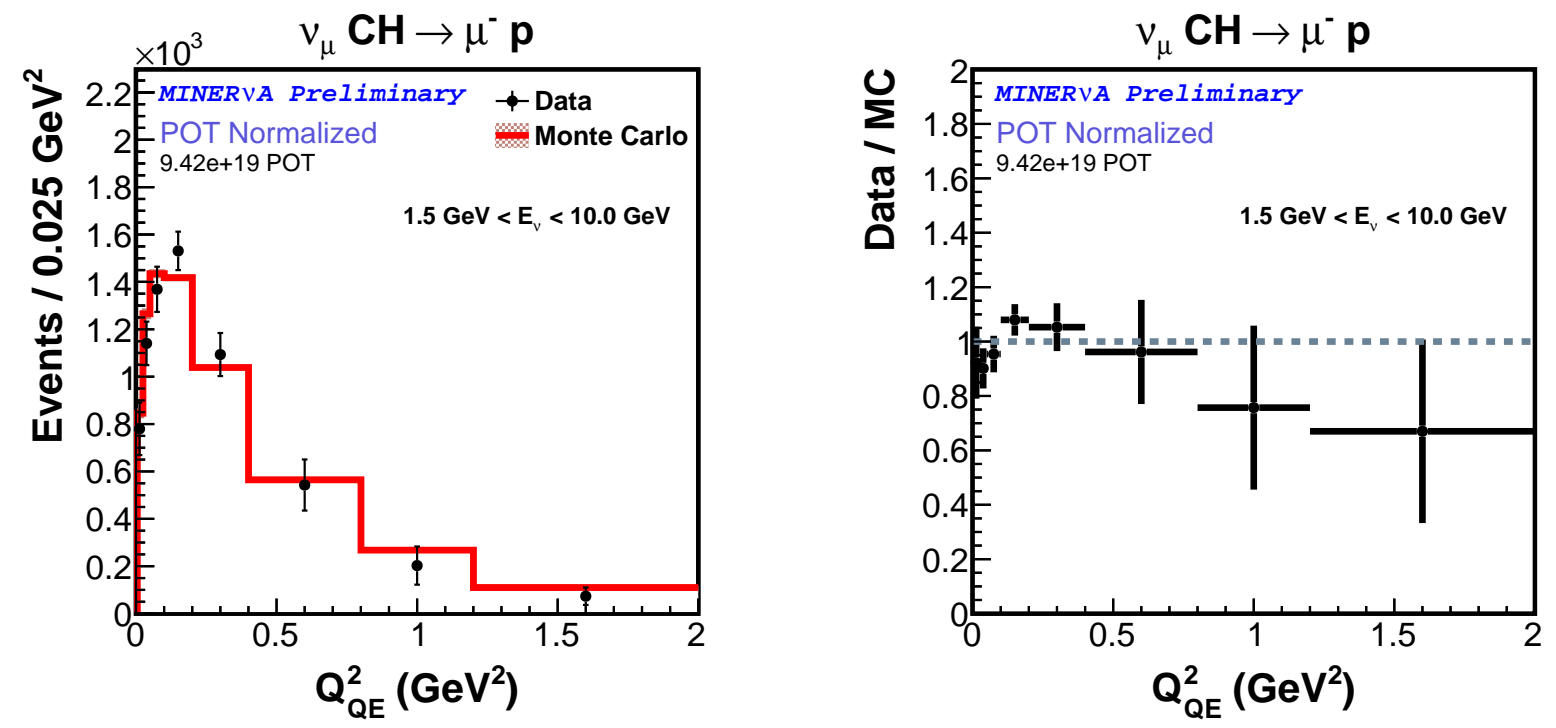

Figure 5.6: Background subtracted, unfolded and efficiency corrected distribution of events in bins of true $Q_{\mathrm{QE}}^{2}$ (left). The ratio between data and MC (right).

\subsubsection{Normalization}

As the last step we convert the corrected distribution into a cross section by dividing the distribution by the number of targets in the fiducial volume (1.65 $\pm 0.02 \times 10^{30}$ neutrons), the flux integral $\left(2.91 \times 10^{-8} / \mathrm{cm}^{2}\right.$ per proton on target $)$ and the width of the $Q_{\mathrm{QE}}^{2}$ bin as shown in equation 5.1. The calculated muon neutrino flux per proton on target (POT) is shown in table C.1. The neutrino energy spectrum can be seen in Figure 4.3. 


\subsection{Systematics errors}

There exist several models and reconstruction parameters that influence the cross section measurement presented here. The uncertainty in the knowledge of these parameters is represented as a systematic error that is estimated by using the so called "many universes" method. This method consists in re-calculating the cross section using different values of a model or reconstruction parameter. The variation is done by an amount that represents the uncertainty $( \pm 1 \sigma)$ of the particular parameter. Each variation is called an universe and all of them can be expresed as:

$$
\left(\frac{d \sigma}{d Q_{\mathrm{QE}}^{2}}\right)_{i, u}=\frac{1}{\Phi_{\nu, u} \times T_{n}} \cdot \frac{1}{\left(\Delta Q_{\mathrm{QE}}^{2}\right)_{i}} \cdot \frac{\sum_{j} U_{i j}^{u}\left[N_{j, u}^{\text {data }}-N_{j, u}^{b g}\right]}{\varepsilon_{i, u}}
$$

Where the only difference from equation 5.1 is the additional index $u$ that represents the result in a different universe and $C V$ stands for Central Value which is the result of the MC simulation without any variation. Notice that the migration matrix, selection efficiency and flux are also re-calculated for each universe. When the variation affects the event selection, the cross section is also re-calculated. For each model or reconstruction parameter, there can be several universes and the covariance matrix and errors are calculated as:

$$
\begin{aligned}
\operatorname{cov}(j, k) & =\frac{1}{N} \sum_{i}\left(N_{i, j}-N_{j}^{C V}\right)\left(N_{i, k}-N_{k}^{C V}\right) \\
\sigma_{j} & =\sqrt{\frac{1}{N} \sum_{i}\left(N_{i, j}-N_{j}^{C V}\right)^{2}}
\end{aligned}
$$

Where $N$ is the number of universes considered.

Sometimes it is useful to calculate the shape component of the uncertainties (shape-only uncertainties). This is done by normalizing each universe by the following factor,

$$
F_{u}^{N o r m}=\frac{A_{C V}}{A_{u}}
$$

Where $A_{C V}$ is the area under the central value distribution and $A_{u}$ is the area under the equivalent distribution in a given universe.

By doing this we remove constant differences between universes. The remaining differences are due to the change in shape of the universes with respect to the central value. We then re-calculate the covariance matrix 5.4 and error values 5.5 to obtain the shape-only version of this quantities. Sections from 5.2.1 to 5.2.5 describe the model parameters considered as sources of uncertainties. We generate 100 universes for each case. 


\subsubsection{Neutrino Flux}

Neutrino flux uncertainties are incorporated into the analysis by means of a re-weighting technique. To generate a distribution in a given universe, we apply the weight for that universe to each simulated interaction as a function of neutrino energy. Uncertainties due to the neutrino flux are divided in three types:

Beam Focus- This category represents the uncertainties due to the proton beam and focusing system of the NuMI beamline such as the alignment and the magnetic field model in the horns. They are estimated to be small at most energies, but are significant (8\%) at the focousing peak.

NA49- Uncertainty due to the hadron production constraint on the flux simulation using data from the NA49 hadron production experiment at CERN.

Beam Tertiary- Uncertainty due to the cascade model predictions of tertiary pion production. This uncertainty can be as high as $30 \%$ in the focusing peak.

The uncertainty on the number of muon neutrino charged current interactions in the MINER $\nu \mathrm{A}$ detector of the three sources listed above are given in Figure 5.7.

\subsubsection{Muon Reconstruction}

Uncertainty due to the muon reconstruction is dominated by the muon energy scale. It is the sum of three effects [65]: the material assay uncertainty in the MINER $\nu \mathrm{A}$ detector (11 MeV ); the $d E / d x$ uncertainty in the MINER $\nu \mathrm{A}$ detector $(30 \mathrm{MeV})$ and the uncertainty in MINOS momemtum measurement. The latter component is $2 \%$ for muons with momentum measured by range in the MINOS near detector, $3.2 \%$ for muons with momentum below $1.0 \mathrm{GeV}$ measured by curvature and $2.1 \%$ for muons with momentum above $1.0 \mathrm{GeV}$ also measured by curvature. The total uncertainty $U_{\text {muon_energy }}$ is the sum in quadrature of the effects listed above. Each universe distribution is filled with simulated interactions where the muon energy is shifted by $U_{\text {muon_energy }} \times f$ ( $f$ is a random number sampled from a normalized gaussian distribution).

\subsubsection{Recoil Reconstruction}

For the recoil energy scale, we considered the uncertainty on the response of protons, pions, neutrons and electromagnetic (EM) particles. The simulated recoil energy that comes from final state protons are varied independently by $3.5 \%$, pions by $5 \%$, neutrons by $10 \%$ and EM particles by $10 \%$. In addition, we treat the cross talk component of the recoil energy in the same way and it is varied by $20 \%$. 


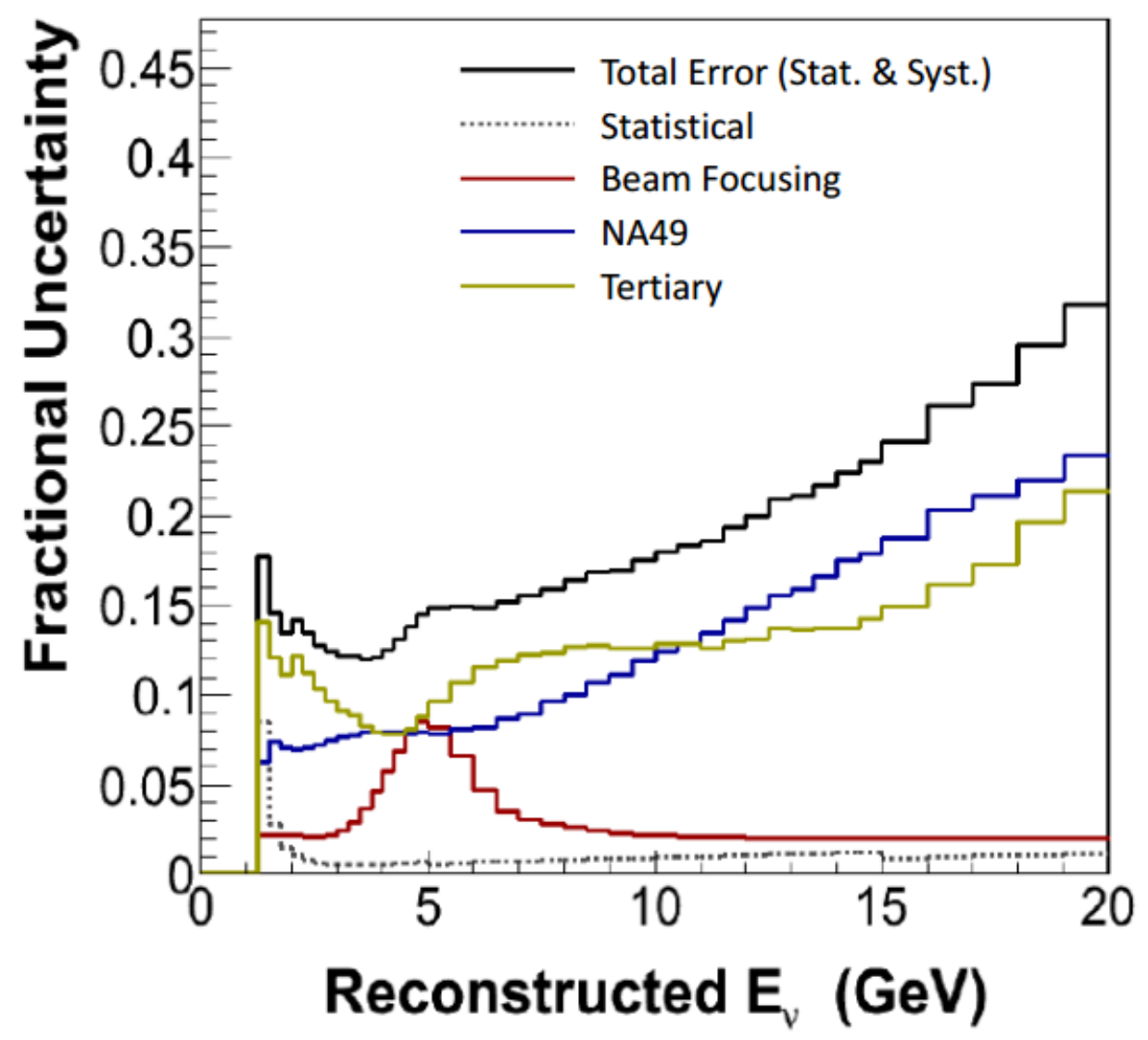

Figure 5.7: Fractional uncertainties on the muon neutrino charged current event rate at the MINER $\nu \mathrm{A}$ detector.

\subsubsection{Primary Interaction}

Primary interaction model parameters change the primary interaction rate prediction. Primary interaction model related systematic uncertainties are measured using the GENIE [48] event reweighting infrastructure, which we use to vary several model parameters by $\pm 1 \sigma$. The parameters are listed in table 5.1

\begin{tabular}{|c|c|}
\hline Parameter & $\pm 1 \sigma$ \\
\hline elastic scattering $M_{A}$ & $25 \%$ \\
resonance normalization & $20 \%$ \\
resonance $M_{A}$ & $20 \%$ \\
resonance $M_{V}$ & $20 \%$ \\
$1 \pi$ non-resonance production & $50 \%$ \\
$2 \pi$ non-resonance production & $50 \%$ \\
\hline
\end{tabular}

Table 5.1: Primary interaction model parameters in GENIE and their $\pm \sigma$ variation to calculate systematics uncertainties in the analysis. 
Parameters that change the signal (CCQE) prediction do not strongly affect the analysis. On the other hand, parameters that change the background prediction will affect the analysis directly through the background subtraction procedure. However, the uncertainty due to them, are reduced by the background constraint procedure.

\subsubsection{Hadron Interaction}

In this analysis, detection of the recoil hadronic system helps to distinguish quasielastic interactions from inelastic background. Therefore, knowledge of the probability for final state particles to interact with the target remnant is desirable and important. These interactions, known also as Final State Interaction (FSI), are estimated by GENIE and its model parameteres can also be varied using the event re-weighting infrastructure. These parameters are listed in table 5.2.

\begin{tabular}{|c|c|}
\hline Parameter & $\pm 1 \sigma$ \\
\hline pion mean free path & $20 \%$ \\
nucleon mean free path & $20 \%$ \\
pion absortion & $30 \%$ \\
pion charge exchange & $50 \%$ \\
pion elastic scattering & $10 \%$ \\
pion inelastic scattering & $40 \%$ \\
pion $\pi$ production & $20 \%$ \\
nucleon absortion & $20 \%$ \\
nucleon charge exchange & $50 \%$ \\
nucleon elastic scattering & $30 \%$ \\
nucleon elastic scattering & $40 \%$ \\
nucleon $\pi$ production & $20 \%$ \\
AGKY hadronization model & $20 \%$ \\
\hline
\end{tabular}

Table 5.2: Hadron interaction (FSI) model parameters in GENIE and their $\pm \sigma$ variation to calculate systematics uncertainties in the analysis.

These parametres impact the event selection by changing the non-vertex recoil energy prediction.

\subsubsection{Other Sources}

There has been considered more sources of uncertainty in the analysis but, due to its smaller impact than the ones already described, they are only listed here. Uncertainty due to the 
unfolding procedure, the mass of the target and the biding energy value used in CCQE formulas 4.6 and 4.5 .

\subsection{Results}

The total cross section in the neutrino energy range between $1.5 \mathrm{GeV}$ and $10 \mathrm{GeV}$ was found to be $\sigma=0.93 \pm 0.01$ (stat) \pm 0.11 (syst) $\times 10^{-38} \mathrm{~cm}^{-2} /$ neutron. A comparison between MC and data differential cross section, $d \sigma / d Q_{\mathrm{QE}}^{2}$, is shown in Figure 5.8 where the MC has been absolute normalized to data. Data points are shown with statistical and all the systematics uncertainties described before. The differential cross section, $d \sigma / d Q_{\mathrm{QE}}^{2}$, values are listed in table C.2 with the associated uncertainties and correlation matrix. The uncertainties per $Q_{\mathrm{QE}}^{2}$ bin divided by its several components are shown in table C.3.
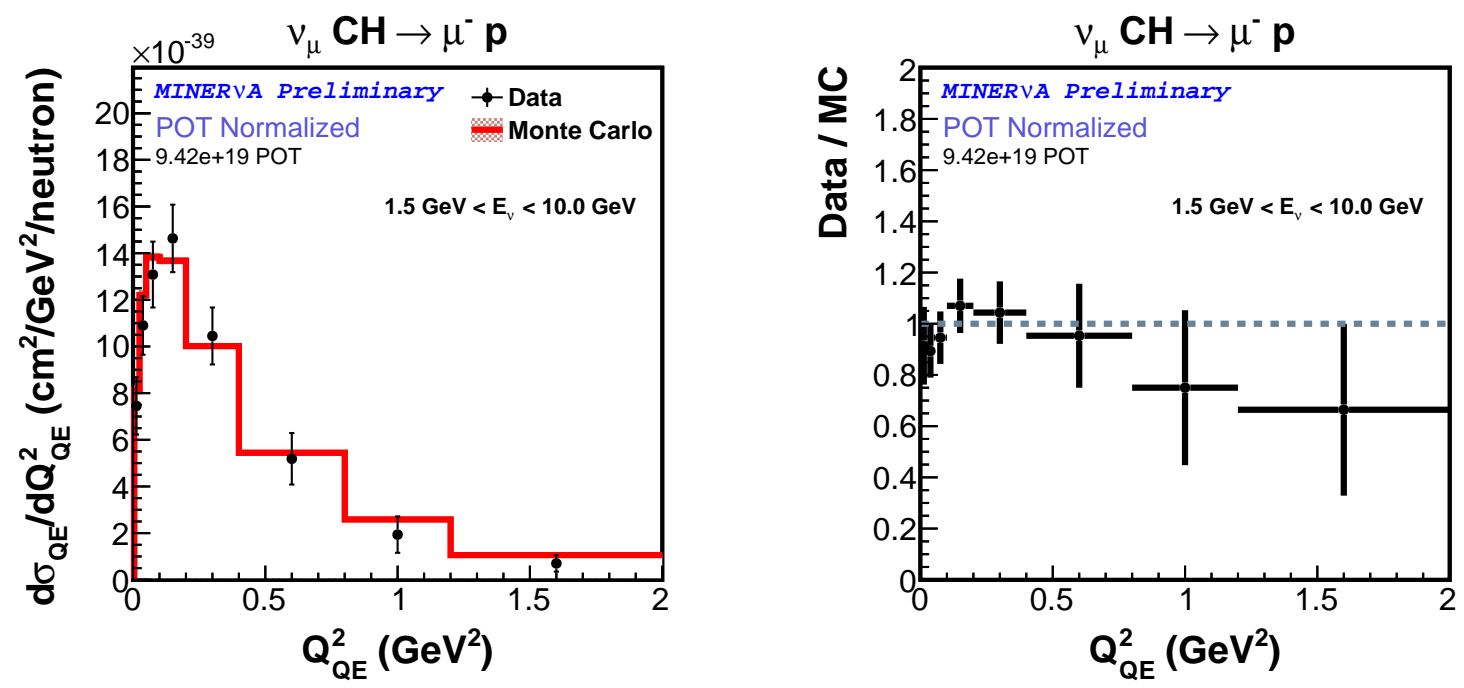

Figure 5.8: Differential cross section in bins of reconstructed $Q_{\mathrm{QE}}^{2}$ as defined in equation 4.6 (left). The ratio between data and MC (right). The MC has been normalized to the number of POT in data (POT Normalization)

Systematics at low $Q_{\mathrm{QE}}^{2}$ are dominated by the flux and the muon reconstruction. In particular, the flux uncertainty do not depend on $Q_{\mathrm{QE}}^{2}$ (flux uncertainties are highly correlated). Thanks to this feature, a shape comparison is preferable since it has lower systematics due to the removal of the flux uncertainties. This effect can be seen when comparing the uncertainty summaries in Figure 5.9 and 5.11. Figure 5.10 shows a shape comparison between data and MC of the differential cross section where data is showed with statistical and the shape component of the uncertainties. The shape comparison of the differential cross section, $d \sigma / d Q_{\mathrm{QE}}^{2}$, values are listed in table C.4 with the associated uncertainties and correlation matrix. 


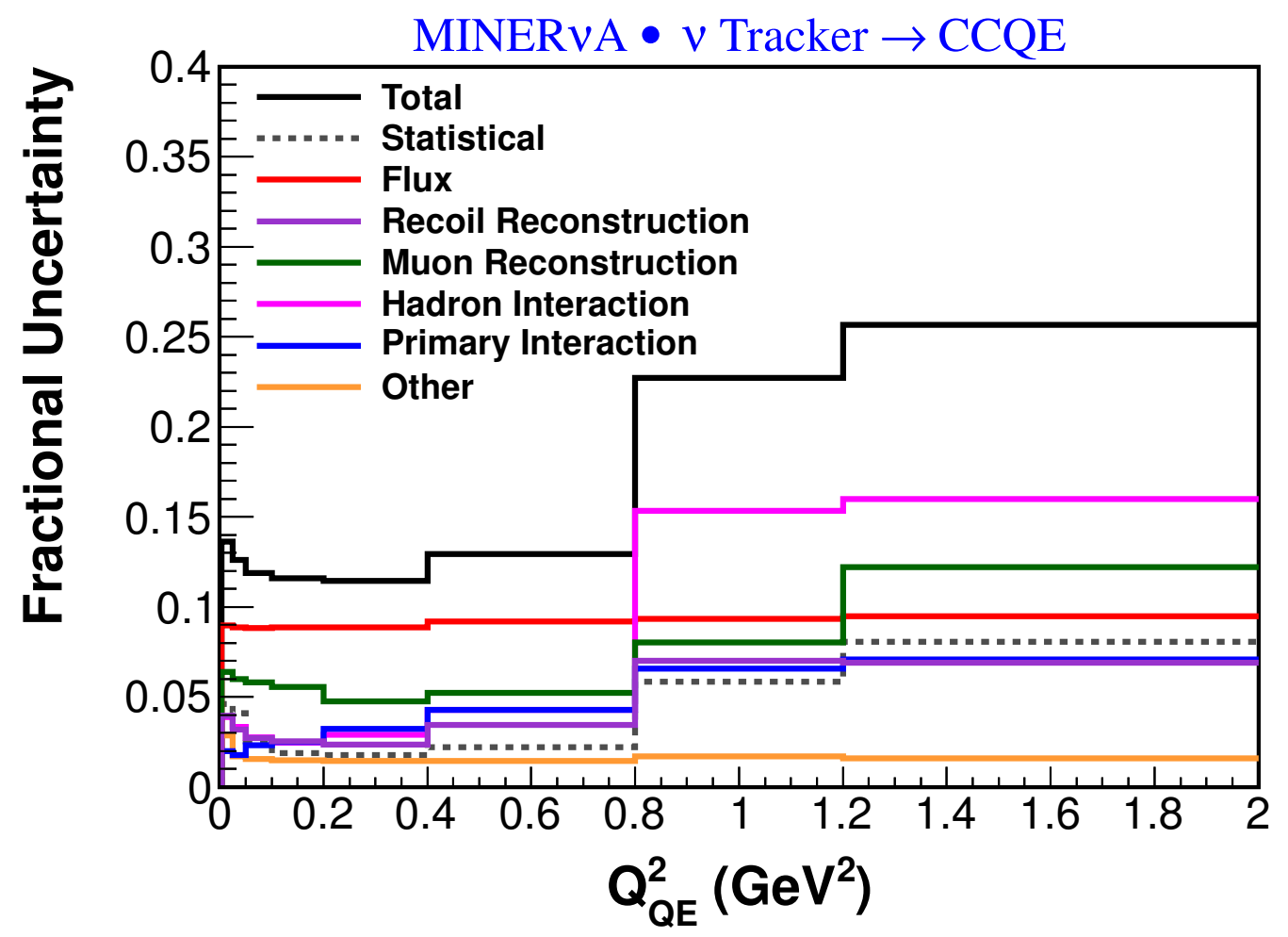

Figure 5.9: Fractional uncertainties for the diferential cross section as function of reconstructed $Q_{\mathrm{QE}}^{2}$.
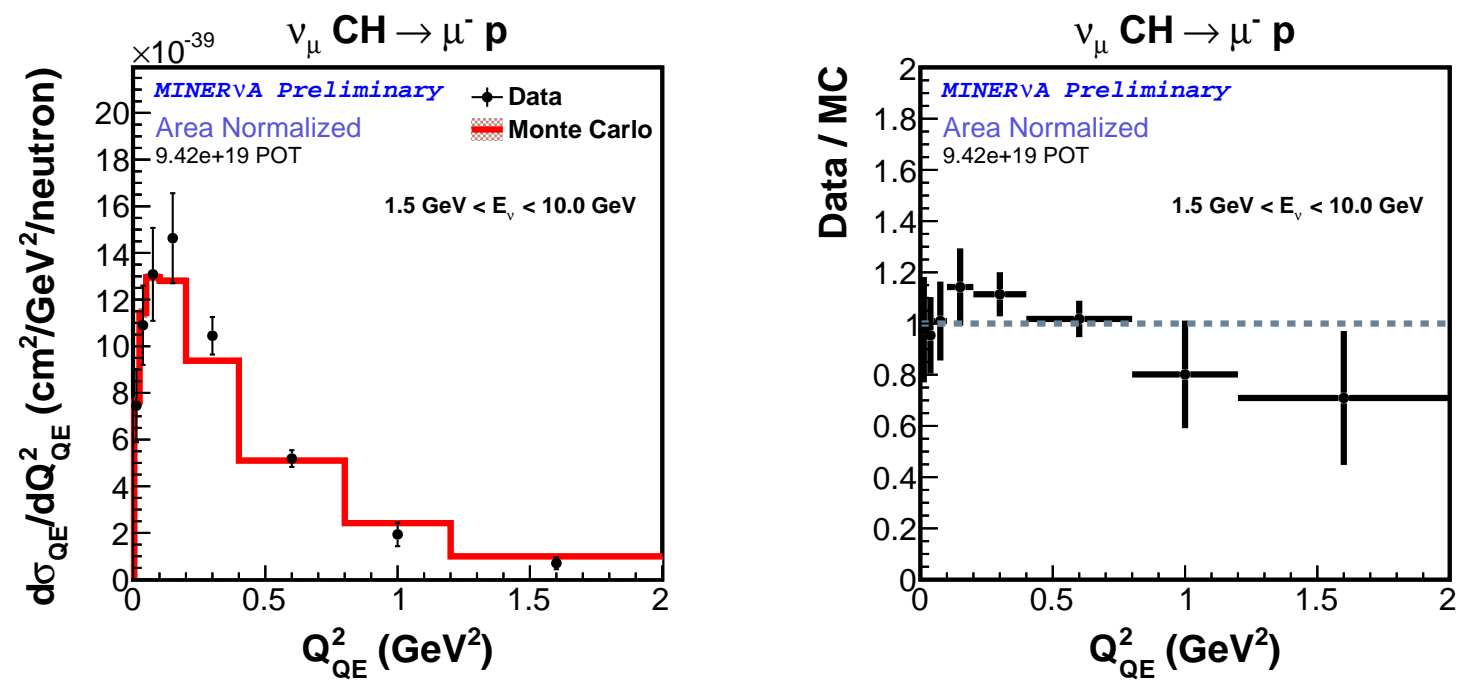

Figure 5.10: Differential cross section in bins of reconstructed $Q_{\mathrm{QE}}^{2}$ as defined in equation 4.6 (left). The ratio between data and MC (right). The MC has been normalized to the total cross section in data (Area Normalization). 


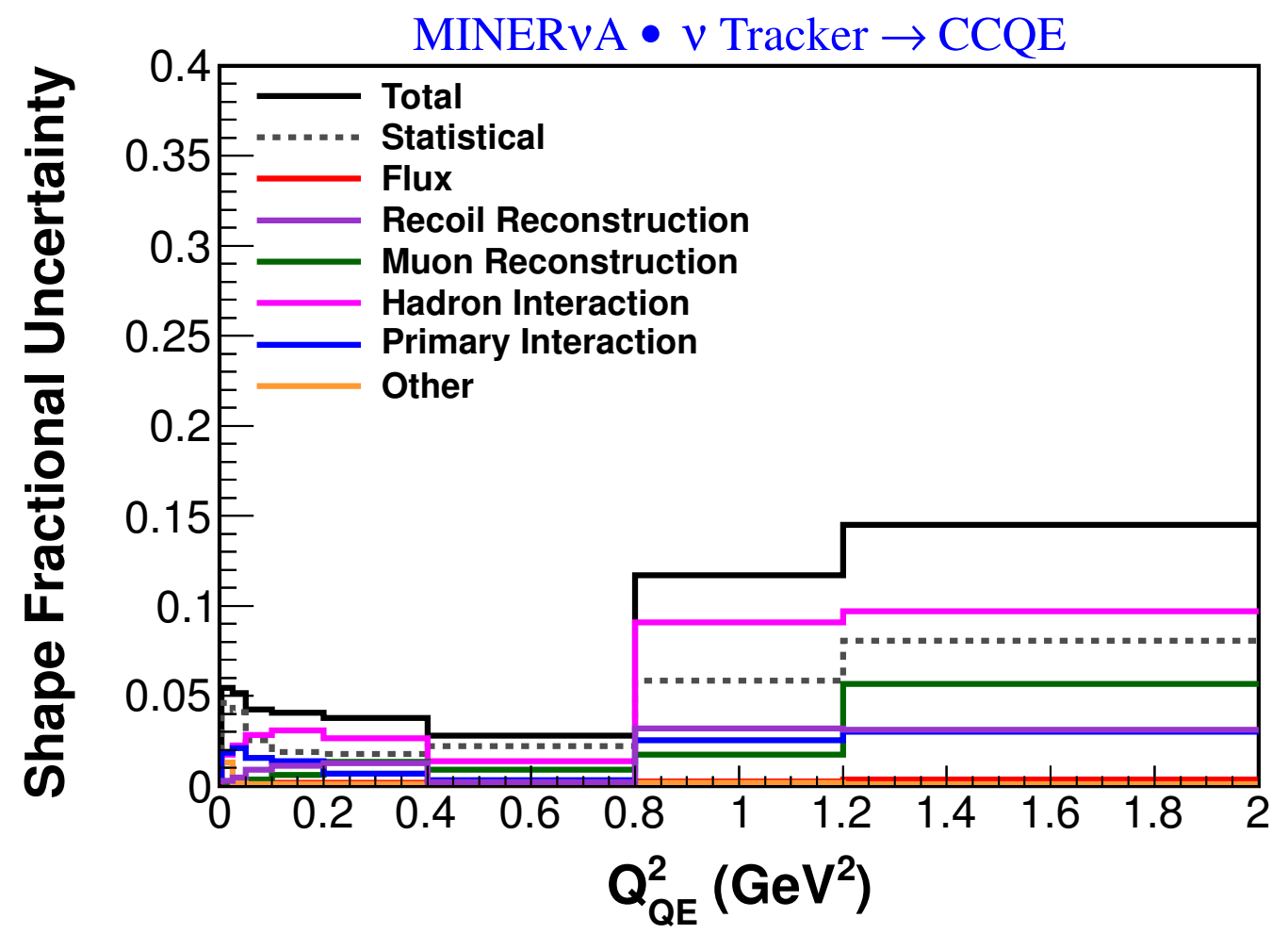

Figure 5.11: Fractional uncertainties for the diferential cross section as function of reconstructed $Q_{\mathrm{QE}}^{2}$. Shape uncertainties.

\subsection{Comparison with Models}

We compare our differential cross section result with the GENIE prediction and the several CCQE models avaliable in the NuWro [57] neutrino interaction generator.

GENIE RFG $M_{A}=\mathbf{0 . 9 9}$ The relativistic fermi gas model with $M_{A}=0.99 \mathrm{GeV}$ implemented by GENIE.

NuWro RFG $M_{A}=\mathbf{0 . 9 9}$ The relativistic fermi gas model with $M_{A}=0.99 \mathrm{GeV}$ implemented by NuWro.

NuWro RFG $M_{A}=\mathbf{1 . 3 5}$ The relativistic fermi gas model with $M_{A}=1.35 \mathrm{GeV}$ implemented by NuWro.

NuWro SF $M_{A}=\mathbf{0 . 9 9}$ The spectral function with $M_{A}=0.99 \mathrm{GeV}$ implemented by NuWro.

NuWro RFG $M_{A}=\mathbf{0 . 9 9}+\mathbf{T E M}$ The relativistic fermi gas model with $M_{A}=1.35 \mathrm{GeV}$ implemented by $\mathrm{NuWro.}$

Together with the value of the predicted and measured differential cross section, a ratio between all of them to the default GENIE prediction (RFG $\left.M_{A}=0.99 \mathrm{GeV}\right)$ is shown in Figure 
5.12 in order to better highlight their differences. From this comparison, it can be seen that the same RFG with $M_{A}=0.99$ model in GENIE and NuWro are not identical but have small differences in the order of $\sim 1 \%$ at values of $Q_{\mathrm{QE}}^{2}$ less than $\sim 0.2 \mathrm{GeV}^{2}$. This is just due to the slightly different value of the binding energy for carbon in the implementantion of the RFG model in both generators.

Since we are interested in a shape comparison between MC predictions and data, because it will remove fully correlated uncertainties such as the flux, we normalize by area all the predictions to the data. Again, a ratio to the GENIE prediction is also calculated in order to highliht the differences between the data and their predictions. Both are shown in Figure 5.13.

The the ratio plot in figure 5.13 shows that the data disfavors models based on the RFG and seems to favor the shape of the curve corresponding to the NuWro RFG $M_{A}=0.99+$ TEM prediction. Notice the importance of the high $Q_{\mathrm{QE}}^{2}$ bins in this comparisons. For values of $Q_{\mathrm{QE}}^{2}$ less than $\sim 0.4 \mathrm{GeV}^{2}$, the data would seem to follow the shape (not the absolute value) of two predictions, NuWro RFG $M_{A}=0.99+$ TEM and NuWro RFG $M_{A}=1.35$. It is at high values of $Q_{\mathrm{QE}}^{2}$ that the two predictions diverge, and the data seems to prefer NuWro RFG $M_{A}=0.99+$ TEM. This is a qualitative comparisons and in order to have a quantitative one, we calculate the $\chi^{2}$ obtained from comparing the differential cross section measured with the different models. This is shown in table 5.3.

\begin{tabular}{|c|c|c|c|c|}
\hline Model & NuWro RFG & NuWro RFG+TEM & RFG & SF \\
\hline$M_{A}$ & 0.99 & 0.99 & 1.35 & 0.99 \\
\hline Absolute $\chi^{2} / D O F$ & 3.5 & 2.4 & 3.7 & 2.8 \\
Shape $\chi^{2} / D O F$ & 4.1 & 1.7 & 2.1 & 3.8 \\
\hline
\end{tabular}

Table 5.3: The $\chi^{2}$ per degree of freedom (DOF) values obtained when the measured differential cross section $d \sigma / d Q_{\mathrm{QE}}^{2}$ is compared with the its different predictions. For the absolute comparisons DOF is eight and seven for the shape comparisons.

The $\chi^{2}$ values confirms that data prefers the shape of the curve corresponding to the NuWro $M_{A}=0.99 \mathrm{GeV}+\mathrm{TEM}$ prediction as stated before. 

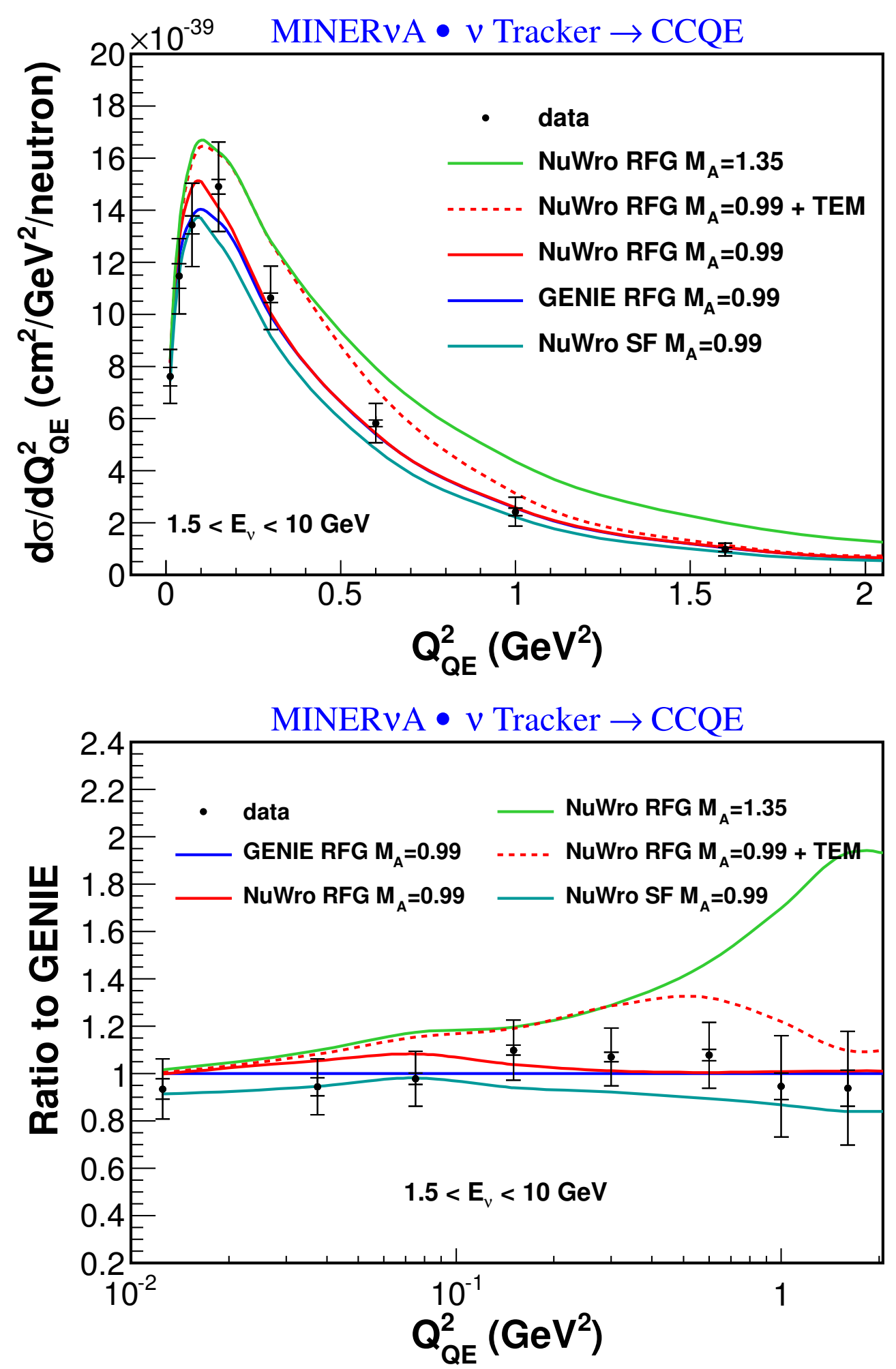

Figure 5.12: Cross section results and an absolute comparison with GENIE and additional models in NuWro. Top plot is a direct comparison. In the bottom plot all cross sections have been divided by GENIE prediction and presented as a function of $Q_{\mathrm{QE}}^{2}$ in a logarithmic scale to show better low $Q_{\mathrm{QE}}^{2}$ values. 

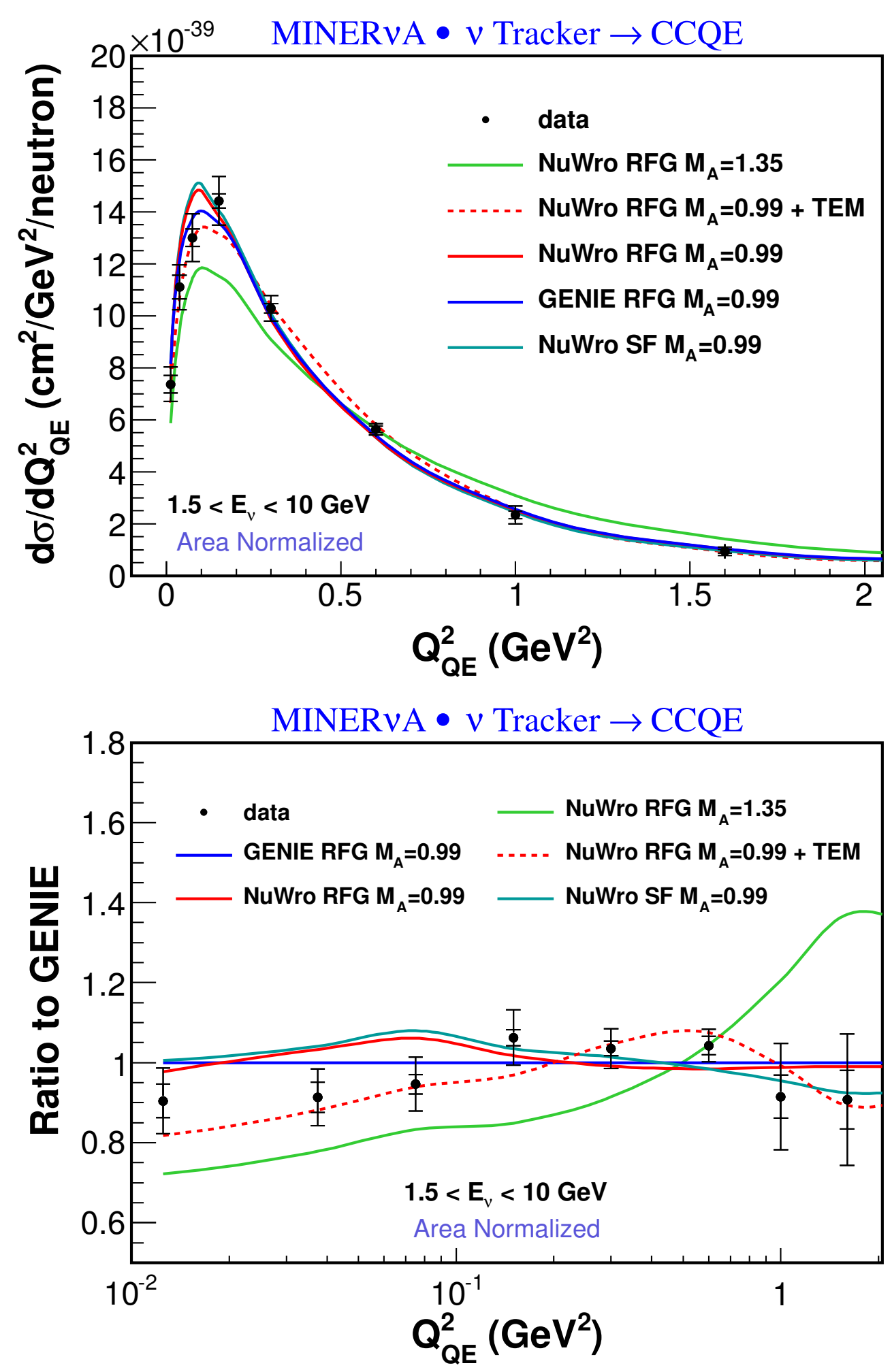

Figure 5.13: Cross section results and an shape comparison with GENIE and additional models in NuWro. Top plot is a direct comparison. In the bottom plot all cross sections have been divided by GENIE prediction and presented as a function of $Q_{\mathrm{QE}}^{2}$ in a logarithmic scale to show better low $Q_{\mathrm{QE}}^{2}$ values. 


\section{Chapter 6}

\section{Conclusions}

In this thesis, we present the first measurement of the single (flux-averaged) differential cross section, $d \sigma / d Q_{Q E}^{2}$, for muon neutrino charged-current quasielastic (CCQE) interactions on a hydrocarbon $(\mathrm{CH})$ target using the MINER $\nu$ A detector in the NuMI neutrino beam at Fermilab. The measurement was restricted to neutrino energies between $1.5 \mathrm{GeV}$ and $10 \mathrm{GeV}$ with an average of $\sim 3.5 \mathrm{GeV}$. The data used in this analysis represents $1 / 4$ of the total data collected by MINER $\nu \mathrm{A}$ in the low-energy configuration of the NuMI neutrino beam.

The selection of muon neutrino CCQE interactions was based on the identification of a negative muon and the requirement of low calorimetric recoil energy separated from the interaction vertex. By looking at the calorimetric recoil energy separeted from the interaction vertex, we include in the measurement CCQE interactions with more than one nucleon in the final state that may be due to correlations between target nucleons. This allow us to compare our results with models containing these effects. In addition, the GENIE-based background prediction of the selected sample was improved by using MINER $\nu$ A's data to calculate correction factors in bins of $Q_{\mathrm{QE}}^{2}$.

We calculated the single differential cross section $d \sigma / d Q_{Q E}^{2}$ of muon neutrino CCQE interactions from our data using this selection and compared it with different MC models avaliable. MINER $\nu$ A's data disfavors models that describe the carbon nucleus as a collection of independent nucleons bound in a nuclear potential (RFG or spectral functions models) that have been avaliable in the neutrino event generators used in this work (GENIE and NuWro) and many others for a long time. It also disfavors MC simulations using the RFG model and a dipole axial-vector form factor mass $M_{A}$ with a higher value $\left(M_{A}=1.35 \mathrm{GeV}\right)$ than the world average $\left(M_{A}=0.99 \mathrm{GeV}\right)$ in an attempt to account for nuclear effects in the scattering off target nucleons inside carbon nucleus. Instead, the data favors a model derived from an observed enhacement of the tranverse part of the electron-nucleus scattering cross section attributed to meson exchange currents in a nucleus originated from multi-nucleon correlations (TEM model) 
that is currently avaliable in the NuWro neutrino event generator.

Future versions of this analysis will include several improvements. Better statistics with the use of the full neutrino data in the low-energy configuration of the NuMI neutrino beam. Systematic uncertainties that are dominated by large flux uncertainties are expected to be significantly reduced by improving flux simulation. The identification of Michel electrons $\left(\pi^{+} \rightarrow \mu^{+} \rightarrow e^{+}\right)$will improve selection efficiencies and sample purities by allowing to reject background events with pions in the final state. Several other measurements with MINER $\nu$ A's data will complement this work, like: comparison with an analysis of CCQE interactions including the final state proton reconstruction; measurement of the double differential cross section, $d^{2} \sigma / d T_{\mu} d \theta_{\mu}$, for a model independent comparison with MC simulations; measurement of absolute CCQE cross section as a function of neutrino energy; and CCQE cross section in different nuclear targets such as $\mathrm{Pb}$ and Fe.

The analysis presented in this thesis was published in [77]. 


\section{Appendix A}

\section{CCQE Candidates Distributions}

In this appendix we show several distributions of the event that have passed all the event selection described in section 4.4. These events are called CCQE candidates.

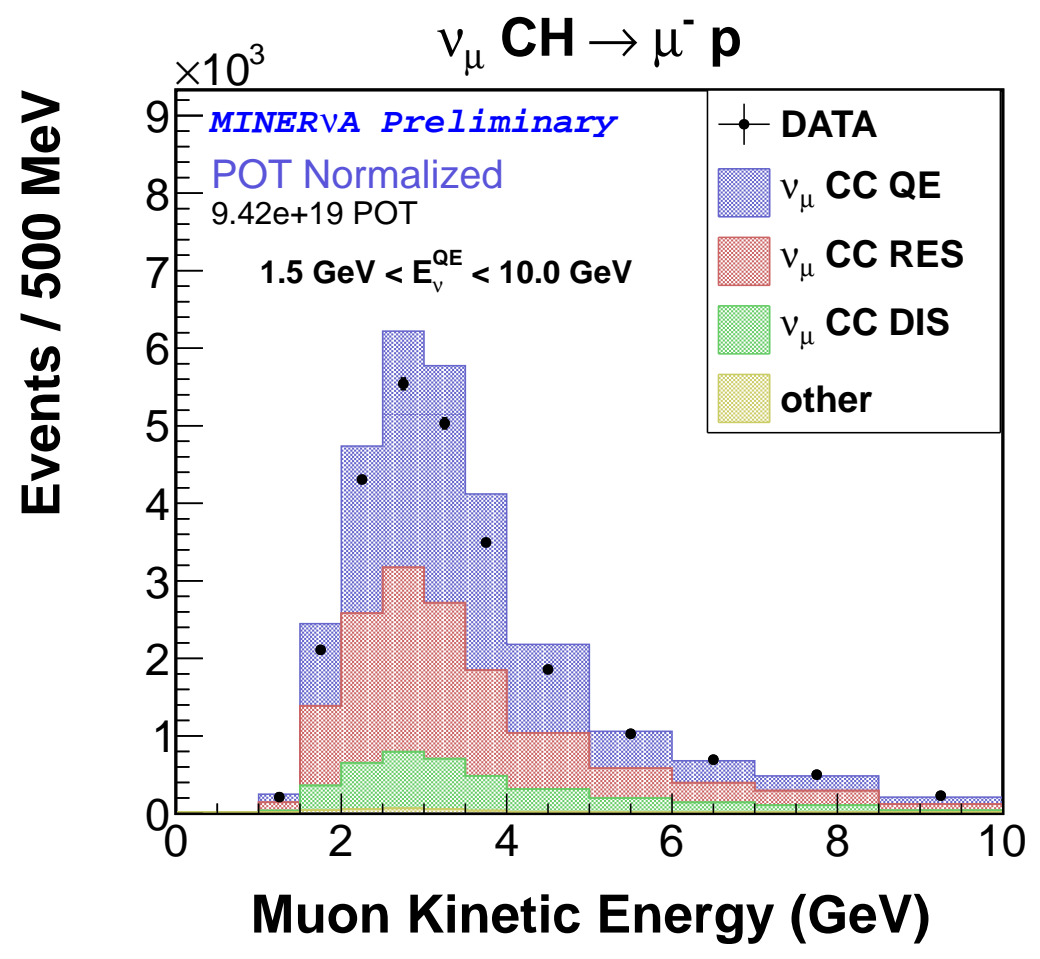

Figure A.1: The reconstructed muon energy distribution of CCQE candidates. 


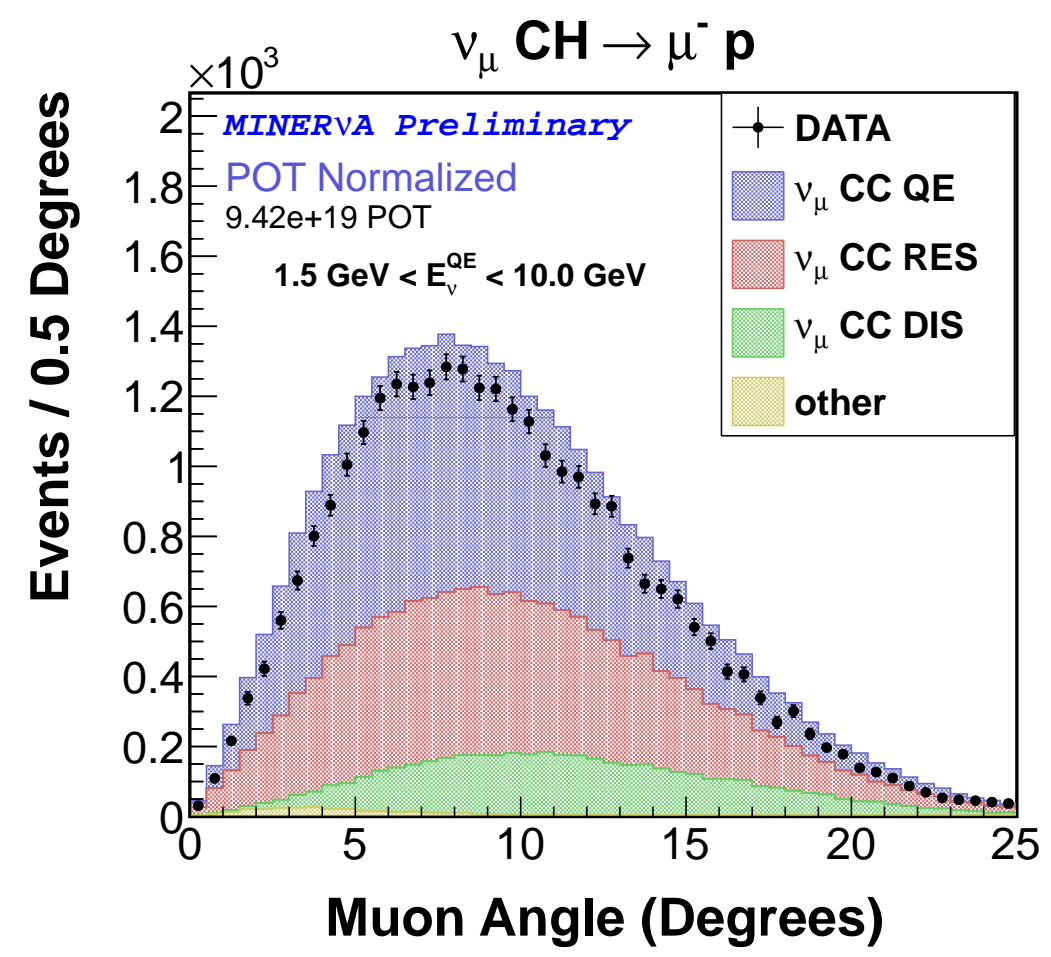

Figure A.2: The reconstructed muon $\theta$ angle distribution of CCQE candidates.
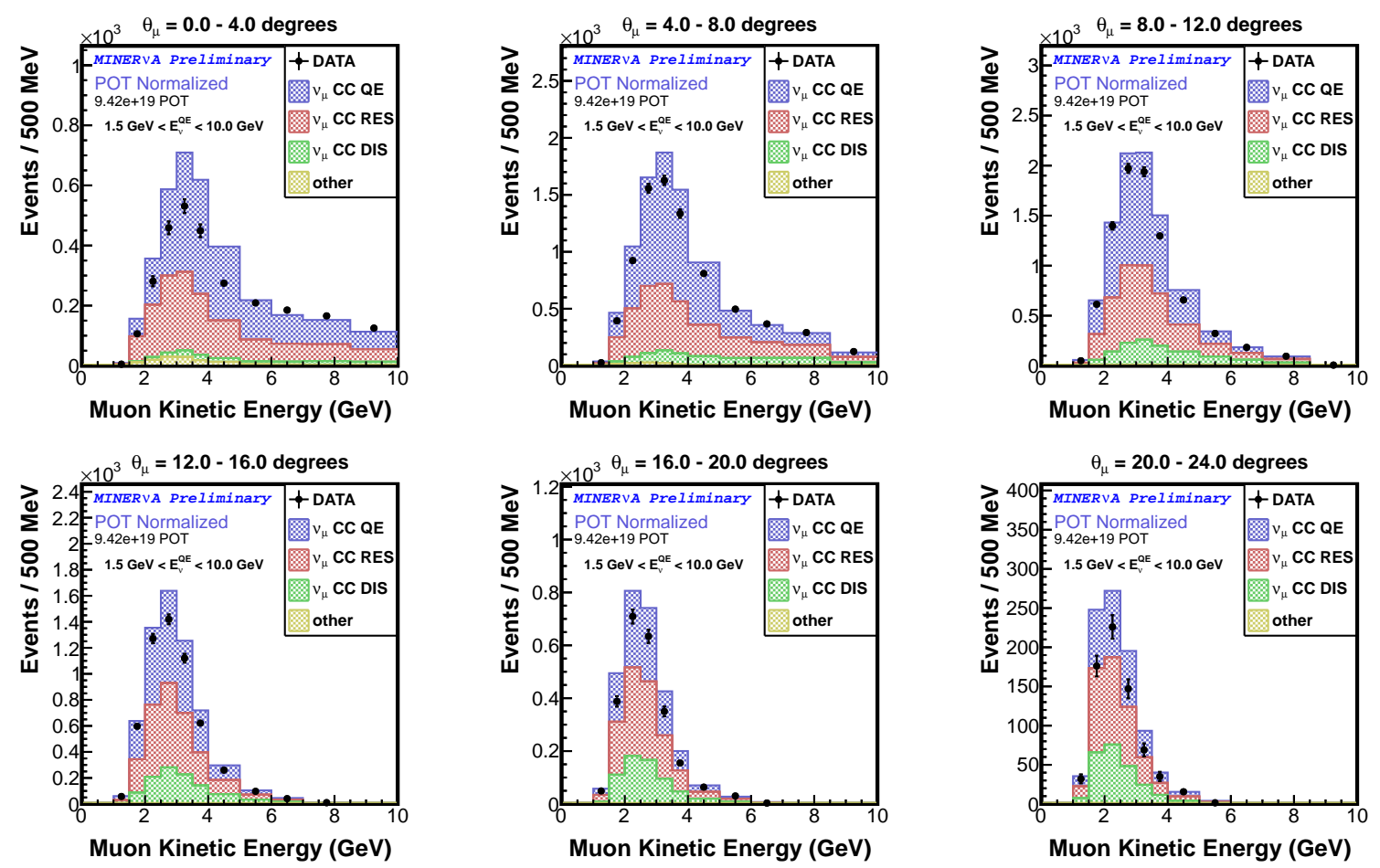

Figure A.3: The reconstructed muon energy distribution in bins of reconstructed muon angle of CCQE candidates. 

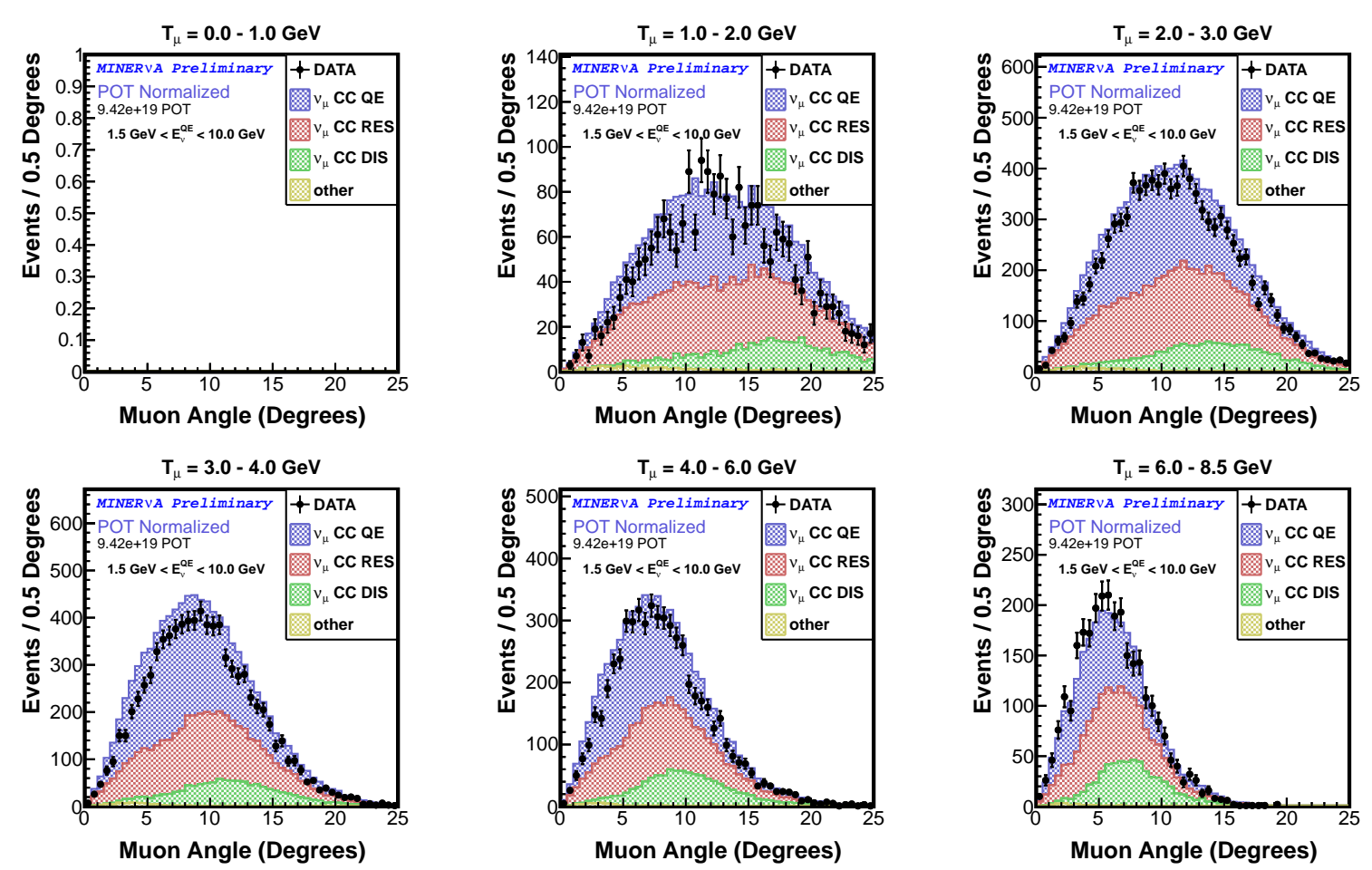

Figure A.4: The reconstructed muon angle distribution in bins of reconstructed muon energy of CCQE candidates.

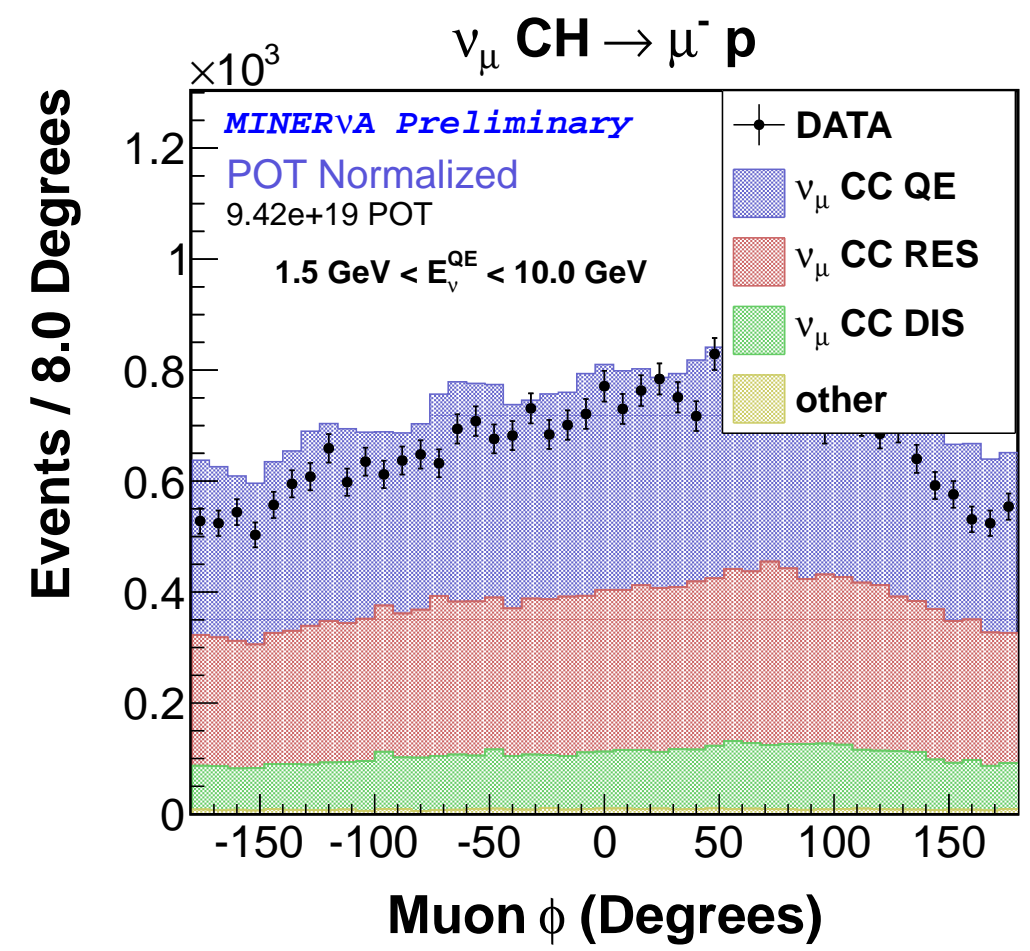

Figure A.5: The reconstructed muon $\phi$ angle distribution of CCQE candidates. 

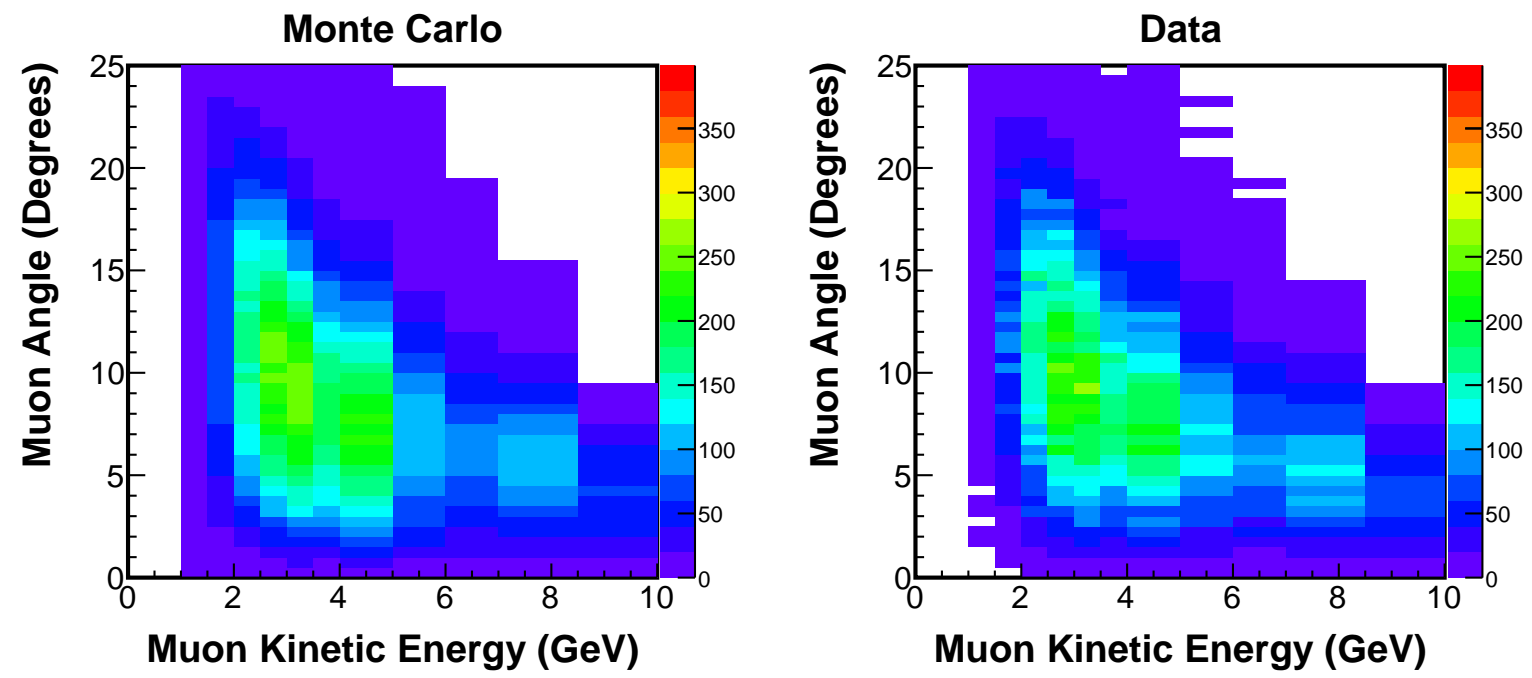

Figure A.6: The reconstructed muon angle vs energy distribution of CCQE candidates. 


\section{Appendix B}

\section{Efficiencies and Purities}

Selection efficiency and sample purity resulting from the event selection procedure in section 4.15. They are defined in equations 4.11 for efficiencies and 4.12.
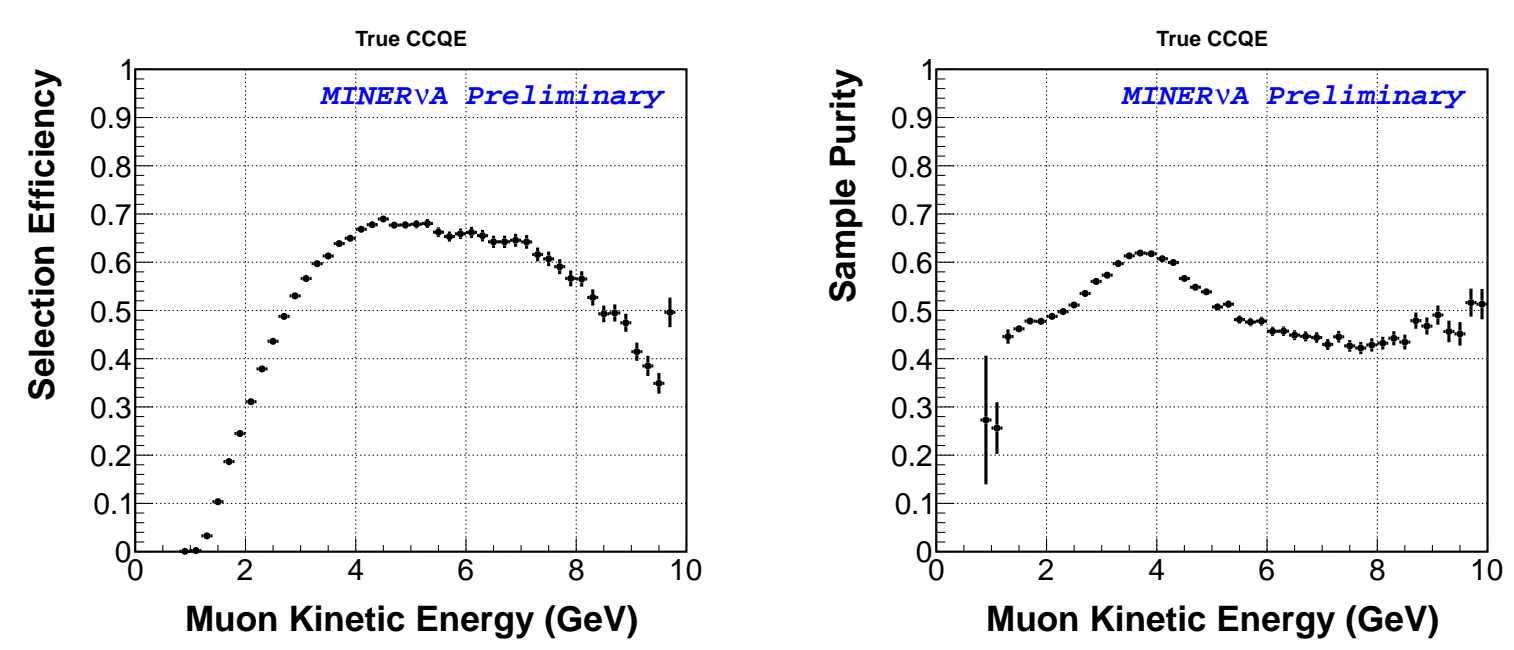

Figure B.1: Selection efficiency and sample purity as a function of generated muon energy. 

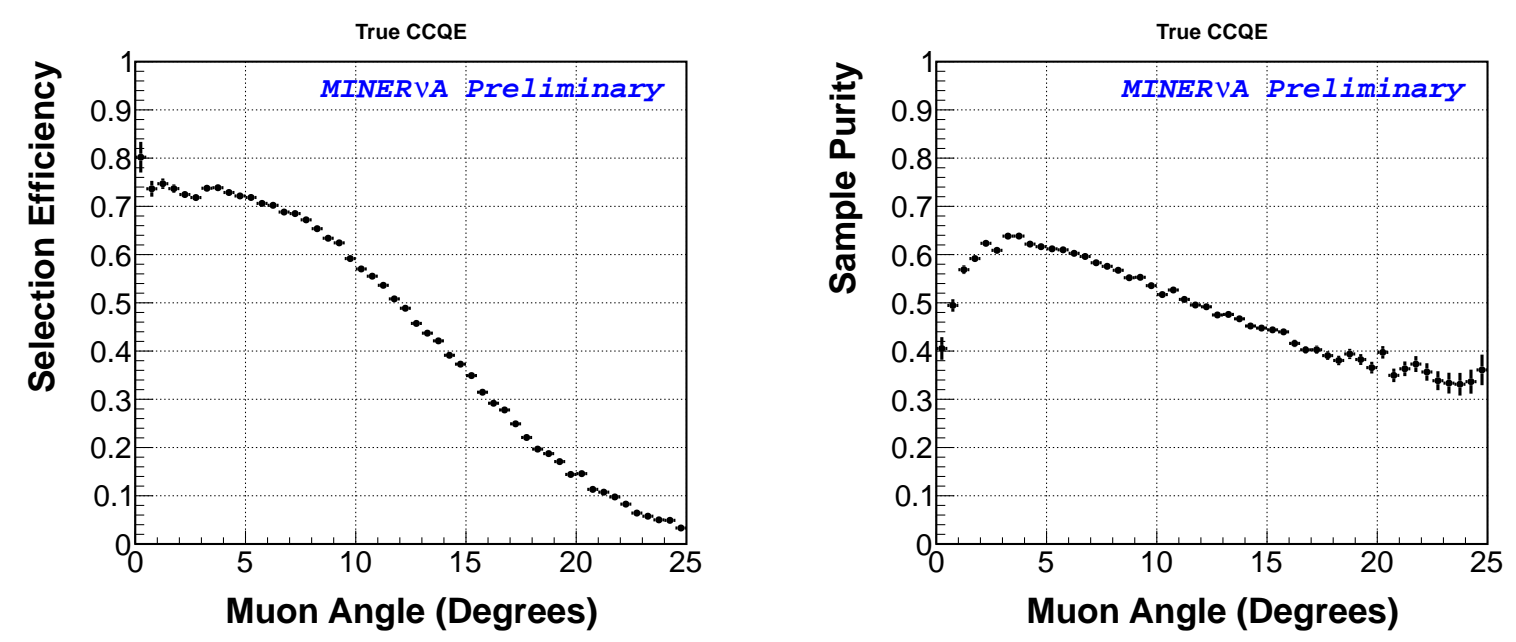

Figure B.2: Selection efficiency and sample purity as a function of generated muon $\theta$ angle.
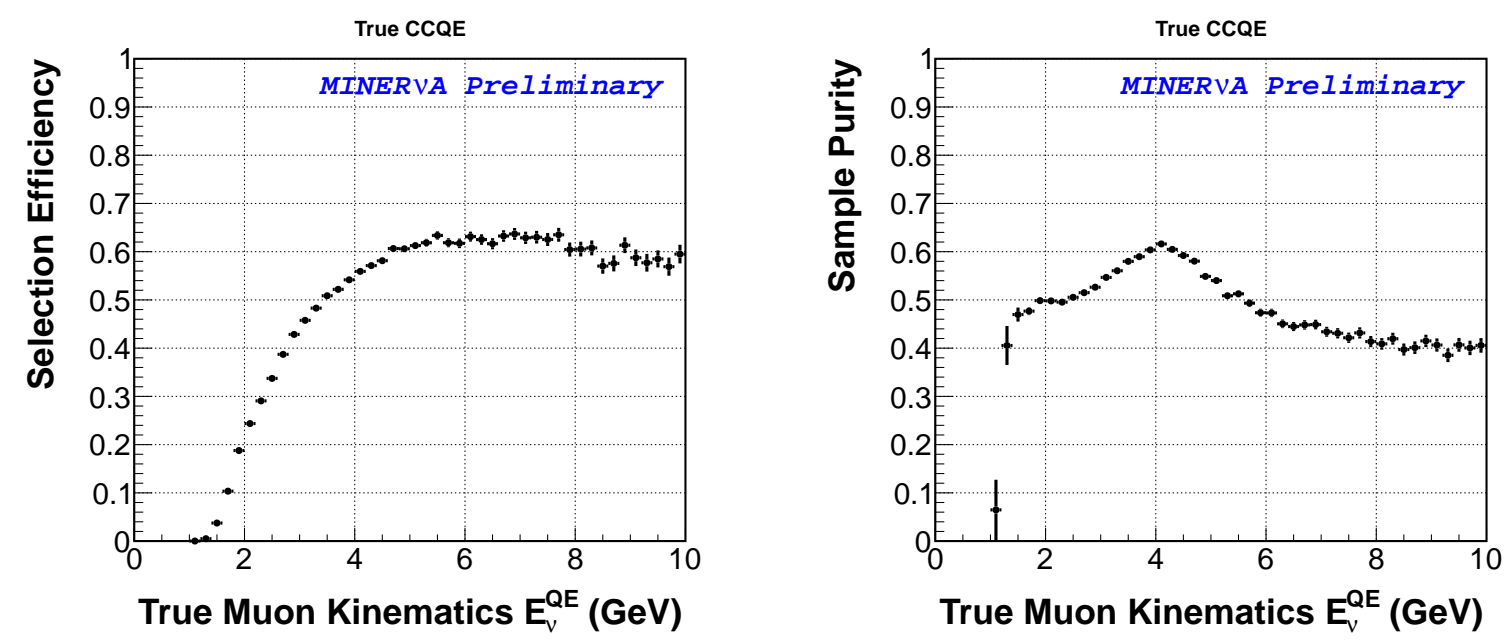

Figure B.3: Selection efficiency and sample purity as a function of $E_{\nu}^{\mathrm{QE}}$ as defined in equation 4.5 using generated muon momentum and angle. 

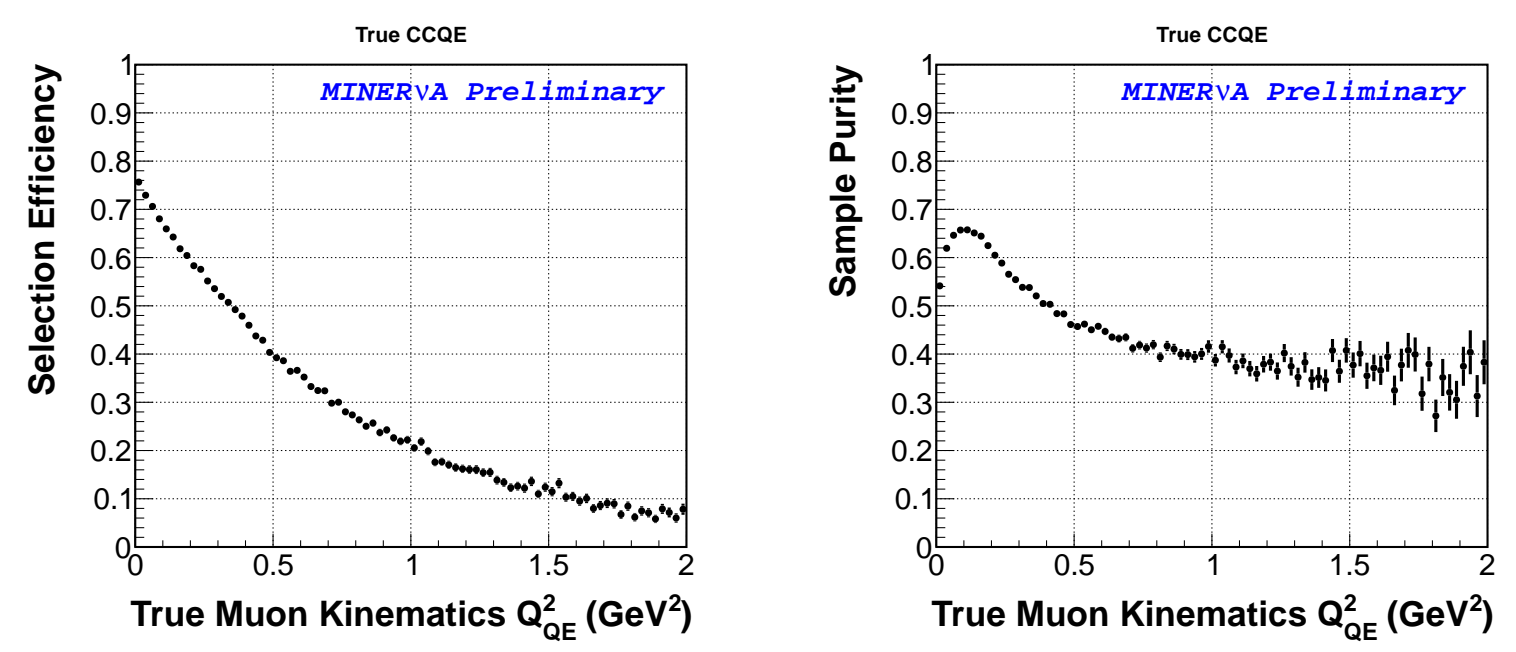

Figure B.4: Selection efficiency and sample purity as a function of $Q_{\mathrm{QE}}^{2}$ as defined in equation 4.6 using generated muon momentum and angle.
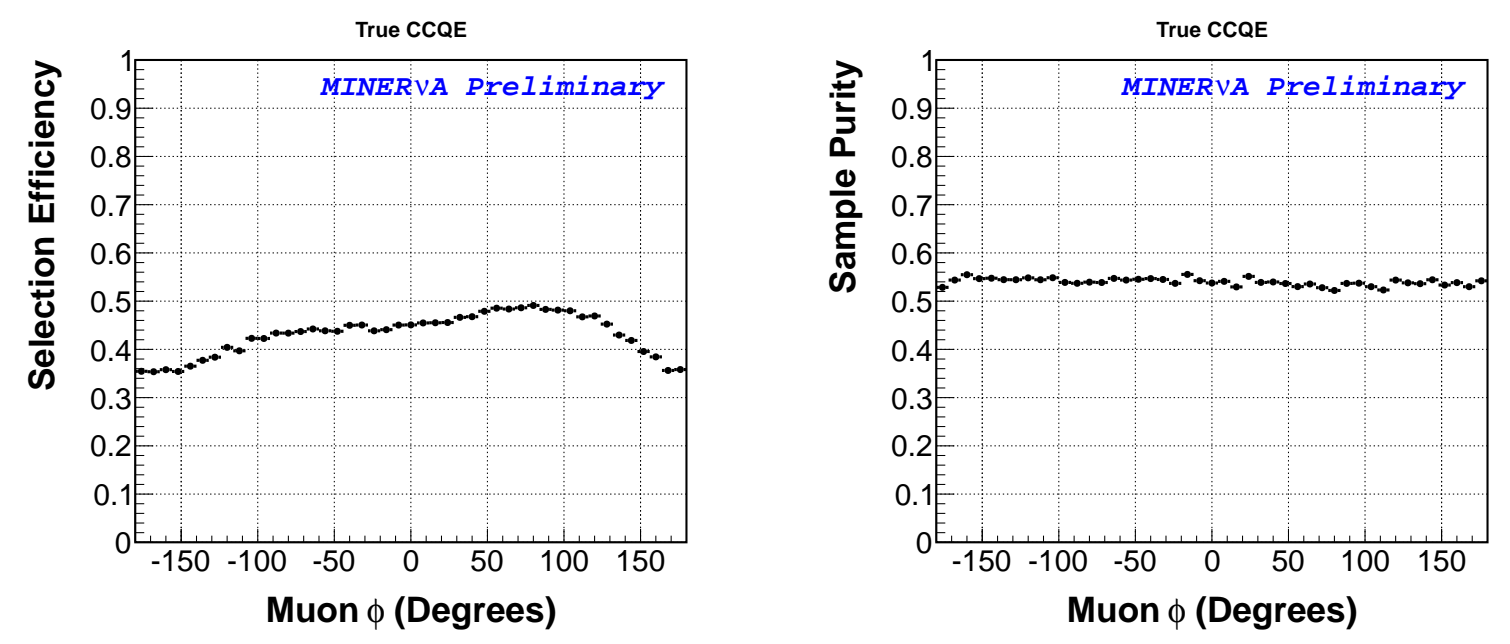

Figure B.5: Selection efficiency and sample purity as a function of generated muon $\phi$ angle. 


\section{Appendix C}

\section{Results}

Tables corresponding to the results presented in chapter 5 .

\begin{tabular}{l|ccccccccc}
\hline$E_{\nu}$ in Bin & $1.5-2$ & $2-2.5$ & $2.5-3$ & $3-3.5$ & $3.5-4$ & $4-4.5$ & $4.5-5$ & $5-5.5$ \\
$\nu_{\mu}$ Flux (neutrinos $/ \mathrm{cm}^{2} / \mathrm{POT}\left(\times 10^{-8}\right)$ & 0.310 & 0.409 & 0.504 & 0.526 & 0.423 & 0.253 & 0.137 & 0.081 \\
\hline$E_{\nu}$ in Bin & $5.5-6$ & $6-6.5$ & $6.5-7$ & $7-7.5$ & $7.5-8$ & $8-8.5$ & $8.5-9$ & $9-9.5$ & $9.5-10$ \\
$\nu_{\mu}$ Flux (neutrinos $/ \mathrm{cm}^{2} / \mathrm{POT}\left(\times 10^{-8}\right)$ & 0.055 & 0.043 & 0.036 & 0.031 & 0.027 & 0.024 & 0.021 & 0.019 & 0.017 \\
\hline
\end{tabular}

Table C.1: The calculated muon neutrino flux per proton on target (POT) for the data included in this analysis. This table correspond to figure 4.3.

\begin{tabular}{|c|c|c|c|c|c|c|c|c|}
\hline$Q_{\mathrm{QE}}^{2}\left(\mathrm{GeV}^{2}\right)$ Bins & $0.0-0.025$ & $0.025-0.05$ & $0.05-0.1$ & $0.1-0.2$ & $0.2-0.4$ & $0.4-0.8$ & $0.8-1.2$ & $1.2-2.0$ \\
\hline Cross-section in bin & 0.761 & 1.146 & 1.343 & 1.490 & 1.063 & 0.582 & 0.242 & 0.097 \\
\hline$\left(10^{-38} \mathrm{~cm}^{2} / \mathrm{GeV}^{2} /\right.$ neutron $)$ & \pm 0.104 & \pm 0.144 & \pm 0.160 & \pm 0.172 & \pm 0.122 & \pm 0.075 & \pm 0.055 & \pm 0.025 \\
\hline \multicolumn{9}{|l|}{$Q_{Q E}^{2}\left(\mathrm{GeV}^{2}\right)$} \\
\hline $0.0-0.025$ & 1.000 & 0.869 & 0.882 & 0.873 & 0.832 & 0.690 & 0.415 & 0.327 \\
\hline $0.025-0.05$ & & 1.000 & 0.905 & 0.917 & 0.882 & 0.727 & 0.457 & 0.357 \\
\hline $0.05-0.1$ & & & 1.000 & 0.945 & 0.928 & 0.751 & 0.455 & 0.356 \\
\hline $0.1-0.2$ & & & & 1.000 & 0.946 & 0.788 & 0.481 & 0.385 \\
\hline $0.2-0.4$ & & & & & 1.000 & 0.865 & 0.600 & 0.514 \\
\hline $0.4-0.8$ & & & & & & 1.000 & 0.756 & 0.741 \\
\hline $0.8-1.2$ & & & & & & & 1.000 & 0.888 \\
\hline $1.2-2.0$ & & & & & & & & 1.000 \\
\hline
\end{tabular}

Table C.2: The measurement of the neutrino differential cross-sections in $Q_{Q E}^{2}$, their total (statistical and systematic) uncertainties, and the correlation matrix for these uncertainties. This table correspond to figure 5.8 . 


\begin{tabular}{cccccccc}
$Q_{Q E}^{2}\left(\mathrm{GeV}^{2}\right)$ & $\mathrm{I}$ & $\mathrm{II}$ & $\mathrm{III}$ & $\mathrm{IV}$ & $\mathrm{V}$ & $\mathrm{VI}$ & Total \\
\hline $0.0-0.025$ & 0.06 & 0.04 & 0.02 & 0.04 & 0.09 & 0.03 & 0.13 \\
$0.025-0.05$ & 0.06 & 0.03 & 0.02 & 0.03 & 0.09 & 0.02 & 0.12 \\
$0.05-0.1$ & 0.06 & 0.03 & 0.02 & 0.03 & 0.09 & 0.02 & 0.12 \\
$0.1-0.2$ & 0.06 & 0.03 & 0.03 & 0.02 & 0.09 & 0.02 & 0.11 \\
$0.2-0.4$ & 0.05 & 0.02 & 0.03 & 0.03 & 0.09 & 0.01 & 0.11 \\
$0.4-0.8$ & 0.05 & 0.03 & 0.04 & 0.04 & 0.09 & 0.01 & 0.13 \\
$0.8-1.2$ & 0.08 & 0.07 & 0.07 & 0.15 & 0.09 & 0.02 & 0.22 \\
$1.2-2.0$ & 0.12 & 0.07 & 0.07 & 0.16 & 0.09 & 0.02 & 0.24 \\
\hline
\end{tabular}

Table C.3: Fractional systematic uncertainties on $d \sigma / d Q_{Q E}^{2}$ associated with (I) muon reconstruction, (II) recoil reconstruction, (III) neutrino interaction models, (IV) final state interactions, (V) flux and (VI) other sources. The rightmost column shows the total fractional systematic uncertainty due to all sources. This table correspond to figure 5.9.

\begin{tabular}{|c|c|c|c|c|c|c|c|c|}
\hline$Q_{\mathrm{QE}}^{2}\left(\mathrm{GeV}^{2}\right)$ Bins & $0.0-0.025$ & $0.025-0.05$ & $0.05-0.1$ & $0.1-0.2$ & $0.2-0.4$ & $0.4-0.8$ & $0.8-1.2$ & $1.2-2.0$ \\
\hline$\%$ of Cross-section & 2.15 & 3.24 & 7.60 & 16.85 & 24.06 & 26.33 & 10.95 & 8.81 \\
\hline in bin & \pm 0.20 & \pm 0.26 & \pm 0.53 & \pm 1.09 & \pm 1.14 & \pm 1.03 & \pm 1.58 & \pm 1.60 \\
\hline \multicolumn{9}{|l|}{$Q_{Q E}^{2}\left(\mathrm{GeV}^{2}\right)$} \\
\hline $0.0-0.025$ & 1.000 & 0.689 & 0.712 & 0.684 & 0.557 & -0.175 & -0.585 & -0.623 \\
\hline $0.025-0.05$ & & 1.000 & 0.745 & 0.770 & 0.653 & -0.211 & -0.631 & -0.694 \\
\hline $0.05-0.1$ & & & 1.000 & 0.840 & 0.793 & -0.212 & -0.736 & -0.787 \\
\hline $0.1-0.2$ & & & & 1.000 & 0.817 & -0.173 & -0.780 & -0.825 \\
\hline $0.2-0.4$ & & & & & 1.000 & -0.129 & -0.752 & -0.795 \\
\hline $0.4-0.8$ & & & & & & 1.000 & -0.142 & 0.060 \\
\hline $0.8-1.2$ & & & & & & & 1.000 & 0.760 \\
\hline $1.2-2.0$ & & & & & & & & 1.000 \\
\hline
\end{tabular}

Table C.4: The measurement of the shape of the neutrino differential cross-sections for $Q_{Q E}^{2}<$ $2.0 \mathrm{GeV}^{2}$, their total (statistical and systematic) uncertainties, and the correlation matrix for these uncertainties. This table correspond to figure 5.11. 


\section{Glossary}

Below is a glossary of experiment names and several terms used in this work:

- CC Chaged Current.

- CP Chage Parity.

- Daya Bay Reactor neutrino experiemtn in Daya Bay, China.

- DIS Deep Inelastic Scattering interaction.

- Double Chooz reactor neutrino experiment in Chooz, France.

- FNAL Fermi National Accelerator Laboratory.

- FSI Final State Interactions.

- J-PARC Japan Proton Accelerator Research Complex.

- K2K KEK to Kamioaka, long-baseline oscillation experiment using a neutrino beam from KEK to Super-K in Japan.

- KEK Accelerator laboratory in Tsukuba, Japan.

- KamLAND Kamioka Liquid scintillator ANtineutrino Detector, reactor neutrino experiment in Japan.

- LSND Liquid Scintillator Neutrino Detector, sterile neutrino experiment at Los Alamos National Laboratory.

- MINER $\nu \mathrm{A}$ Main Injector Experiment for $\nu$-A, neutrino scattering experiment in the NuMI beamline at FNAL.

- MiniBooNE Mini Booster Neutrino Experiment, short-baseline neutrino oscillation experiment using a Cherenkov detector in the Booster neutrino beamline at FNAL. 
- MINOS Main Injector Neutrino Oscillation Search, neutrino oscillation experiment in the NuMI beamline at FNAL.

- NC Neutral Current.

- NOMAD Neutrino Oscillation MAgnetic Detector, neutrino oscillation experiment at CERN.

- NuMI Neutrino at the Main Injector, neutrino beamline at FNAL using the Main Injector.

- PMT Photomultiplier tube.

- POT Protons on Target.

- QE Quasi-Elastic interaction.

- RENO Reactor Experiment for Neutrino Oscillations, reactor neutrino experiment in South Korea.

- RES Resonant one pion production interactions.

- RFG Relativistic Fermi Gas model of the nucleus.

- SF Spectral Functions.

- Super-K Super-Kamiokande, water Cherenkov detector in the Kamiokande mine in Japan.

- T2K Tokai to Kamiokande, neutrino oscillation experiment using the JPARC beam in Japan.

- TEM Transfer Enhancement Model. 


\section{Bibliography}

[1] von Bayer, O. Hahn, L. Meitner, Phys. Zeitschrift, 12, p. 378 (1911).

[2] C.D. Ellis, B.A. Wooster, The average energy of desintegration of Radium E, Proc. Roy. Soc. A 117, 109-123 (1927).

[3] W. Pauli, letter sent to the Tubingen conference, Dec. 1930.

[4] J. Chadwick, Possible existence of a neutron, Nature 129, 12 (1932).

[5] E. Fermi, Ricercha Scient. 2, 12 (1933).

[6] F.Perrin, Comptes rendues 197, 1624 (1933).

[7] E. Fermi, Z. Physik, 88, 161 (1934).

[8] F. Reines and C. L. Cowan, The neutrino, Nature 178, 446 (1956).

[9] G. Danby et al., Observation of high-energy neutrino reactions and the existence of two kinds of neutrinos, Phys. Rev. Lett. 9, 36 (1962).

[10] Gargamelle Neutrino Collaboration: F.J. Hasert et al., Observation of neutrino-like interactions without muon or electron in the Gargamelle neutrino experiment, Phys. Lett. B 46, 138 (1973).

[11] DONUT COllaboration: K. Kodama et al., Observation of tau neutrino interactions, Phys. Lett. B 504, 218 (2001).

[12] R. Davis,D.S. Harmer, and K.C. Hoffman, Search for neutrinos from the sun, Phys. Rev. Lett. 20, 1205 (1968).

[13] V.N. Gribov and B. Pontecorvo, Neutrino astronomy and lepton charge, Phys. Lett. B 28, 493 (1969).

[14] B. Pontecorvo, Sov. Phys. JETP 34, 172 (1958). 
[15] Z. Maki, M. Nakagawa and S. Sakata, Remarks on the Unified Model of Elementary Particles, Prog. Theor. Phys. 28, 870 (1962).

[16] Super-Kamiokande Collaboration: Y. Fukuda et al., Evidence for oscillation of atmospheric neutrinos, Phys. Rev. Lett. 81, 1562 (1998).

[17] SNO Collaboration: Q.R. Ahmad et al., Direct evidence for neutrino flavor transformation from neutral-current interactions in the Sudbury Neutrino Observatory, Phys. Rev. Lett. 89, 01131 (2002).

[18] KamLAND Collaboration: K. Eguchi et al., First results from KamLAND: Evidence for reactor anti-neutrino disappearance, Phys. Rev. Lett. 90, 021802 (2003).

[19] K2K Collaboration: M.H. Ahn et al., Measurement of neutrino oscillation by the K2K experiment, Phys. Rev. D 74, 072003 (2006).

[20] MINOS Collaboration: D.G. Michael et al., Observation of Muon Neutrino Disappearance with the MINOS Detector in the NuMI Neutrino Beam, Phys. Rev. Lett. 97, 191801 (2006).

[21] ALEPH Collaboration: D. Decamp et al., Determination of the Number of Light Neutrino Species, Phys. Lett. B 231, 519 (1989).

[22] Delphi Collaboration: P.A. Aarnio et al., Measurement of the Mass and Width of the Z0 Paricle from Multi-Hadronic Final States Produced in the e+ e- Annihilation, Phys. Lett. B 231, 539 (1989).

[23] L3 Collaboration:B. Adeva et al., A Determination of the Properties of the Neutral Intermediate Vector Boson Z0, Phys. Lett. B 231, 509 (1989).

[24] OPAL Collaboration: M.Z. Akrawy et al., Measurement of the Z0 Mass and Width with the OPAL Detector at LEP, Phys. Lett. B 231, 530 (1989).

[25] C.S. Wu et al., Phys. Rev. 105, 1413 (1957).

[26] M. Goldhaber, L. Grodzins, and A.W. Sunyar, Helicity of neutrinos, Phys. Rev. 109, 1015 (1958).

[27] C. W. Kim, Neutrino Physics: Fundamentals of Neutrino Oscillations, Proceedings J. Korean Phys. Soc., arXiv:9607.391 [hep-ph] (1996). 
[28] T2K Collaboration: K. Abe et al., Indication of Electron Neutrinos Appearance from an Accelerator-Produced Off-Axis Muon Neutrino Beam, Phys. Rev. Lett. 107, 041801 (2011).

[29] MINOS Collaboration: P. Adamson et al., Improved Search for Muon-Neutrino to Electron-Neutrino Oscillations in MINOS, Phys. Rev. Lett. 107, 181802 (2011).

[30] Daya Bay Collaboration: F. P. An et al., Observation of Electron-Antineutrino Disappearance at Daya Bay, Phys. Rev. Lett. 108, 171803 (2012).

[31] Daya Bay Collaboration: F. P. An et al., Indication of Reactor $\bar{\nu}_{e}$ Disappearance in the Double Chooz Experiment, Phys. Rev. Lett. 108, 131801 (2012).

[32] Reno Collaboration: J. K. Ahn et al., Observation of Reactor Electron Antineutrinos Disappearance in the RENO experiment, Phys. Rev. Lett. 108, 191802 (2012).

[33] C. Athanassopoulos et al., Candidate Events in a Search for $\bar{\nu}_{\mu} \rightarrow \bar{\nu}_{e}$ Oscillations, Phys. Rev. Lett. 75, 2650 (1995).

[34] MiniBooNE Collaboration: A. A. Aguilar-Arevalo et al., Event Excess in the MiniBooNE Search for $\bar{\nu}_{\mu} \rightarrow \bar{\nu}_{e}$ Oscillations, Phys. Rev. Lett. 105, 181801 (2010).

[35] A. W. Thomas and W. Weise, The Structure of the Nucleon, Wiley- VCH, Berlin, (2001).

[36] 't Hooft, G., Phys. Lett. B 37, 195 (1971).

[37] J. Beringer et al. (Particle Data Group), Phys. Rev. D 86, 010001 (2012).

[38] ATLAS Collaboration, Observation of a new particle in the search for the Standard Model Higgs boson with the ATLAS detector at the LHC, Phys. Lett. B 716 1-29, arXiv:1207.7214 [hep-ex] (2012).

[39] CMS Collaboration, Observation of a new boson at a mass of $125 \mathrm{GeV}$ with the CMS experiment at the LHC, Phys. Lett. B 716 30, arXiv:1207.7235 [hep-ex] (2012).

[40] A. Salam, Proc. of the 8th Nobel Symposium on Elementary Particle Theory, Relativistic Groups and Analyticity, Stockholm, Sweden, edited by N. Svartholm, p. 367-377 (1968).

[41] S. Weinberg, A Model of Leptons, Phys. Rev. Lett., 19, 1264-1266, (1967).

[42] J. A. Formaggio, G. P. Zeller, From eV to EeV: Neutrino cross sections across energy scales, Rev. Mod. Phys. 84, 1307 (2012). 
[43] D. Casper, The Nuance neutrino physics simulation, and the future. Nucl. Phys. B, Proc. Suppl. 112, 161 (2002).

[44] C. H. Llewellyn Smith, Neutrino reactions at accelerator energies, Phys. Rept. 3261 (1972).

[45] A. Bradford, A. Bodek, H. Budd and J. Arrington, A New Parametrization of the Nucleon Elastic Form Factors, Nucl. Phys. Proc. Suppl. 159, 127 (2006).

[46] M. Day and K. S. McFarland, Differences in Quasi-Elastic Cross-Sections of Muon and Electron Neutrinos, Phys. Rev. D 86, 053003 (2012).

[47] R. A. Smith and E. J. Moniz, Neutrino reaction on nuclear targets, Nucl. Phys. B 43, 605 (1972).

[48] C. Andreopoulos, A. Bell, D. Bhattacharya, F. Cavanna, J. Dobson, S. Dytman, H. Gallagher, P. Guzowski, R. Hatcher, P. Kehayias, A. Meregaglia, D. Naples, G. Pearce, A. Rubbia, M. Whalley, T. Yang, The GENIE Neutrino Monte Carlo Generator, Nucl. Instrum. Meth. A 614, 87-104, (2010).

[49] Bernard, V., et al., Axial structure of the nucleon: topical review, J. Phys. G 28, R1 (2002).

[50] K2K Collaboration: R. Gran, et al., Measurement of the quasielastic axial vector mass in neutrino interactions on oxygen, Phys. Rev. D 74, 052002 (206).

[51] X. Espinal and F. Sanchez, Measurement of the axial vector mass in neutrino-carbon interactions at K2K, AIP Conf. Proc. 967117 (2007)

[52] M. Dorman, Preliminary results for CCQE scattering with the MINOS near detector, AIP Conf. Proc. 1189133 (2009)

[53] Aguilar-Arevalo, A. A., et al., First Measurement of the Muon Neutrino Charged Current Quasielastic Double Differential Cross Section, Phys. Rev. D 81, 092005 (2010).

[54] V Lyubushkin et al., A Study of Quasi-elastic muon neutrino and antineutrino scattering in the NOMAD experiment, Eur. Phys. J. C 63, 355-381, (2009).

[55] R. Subedi et al., Probing Cold Dense Nuclear Matter, Science 320, 1476, (2008).

[56] A. Bodek, H.S. Budd and M. E. Christy, Neutrino Quasielastic Scattering on Nuclear Targets: Parametrizing Transverse Enhancement (Meson Exchange Currents), Eur. Phys. J. C 711726 (2011). 
[57] T. Golan, C. Juszczak, J. T. Sobczyk, Effects of final-state interactions in neutrino-nucleus interactions, Phys. Rev. C 86, 015505 (2012).

[58] D. Rein and L.M. Sehgal, Neutrino Excitations of Baryon Resonances and Single Pion Production, Annals of Physics 133, 79 (1981).

[59] M. Gluck, E. Reya and A. Vogt, Dynamical parton distributions revisited, Eur. Phys. J., C5, 461-470 (1998).

[60] A. Bodek and U.K. Yang, Higher twist, $\Xi_{\omega}$ scaling, and effective LOPDFs for lepton scattering in the few GeV region, J. Phys., G29, 1899-1960 (2003).

[61] SciBooNE Collaboration: Nakajima, Y., et al., Phys. Rev. D 83, 012005 (2011).

[62] J. Hylen et al., NuMI Technical Design Handbook, Internal NuMI report (2003).

[63] R. M. Zwaska, Accelerator Systems and Instrumentation for the NuMI Neutrino Beam, PhD thesis University of Texas at Austin, (2005).

[64] FLUKA: A. Ferrari, P.R. Sala, A. Fasso, and J. Ranft, CERN-2005-10, INFN/TC 05/11, SLAC-R-773 (2005).

[65] Minerva Collaboration: L. Aliaga et. al.; Design, Calibration, and Performance of the MINERvA Detector, Nucl. Inst. Meth. (to be published); arXiv:1305.5199 [hep-ex].

[66] http://minerva-docdb.fnal.gov:8080/cgi-bin/ShowDocument?docid=2169.

[67] Minerva Collaboration: G. N. Perdue et. al., The MINERvA Data Acquisition System and Infrastructure, Nucl. Instrum. Meth. A 694 179-192 (2012).

[68] J. Palomino, First Measurement of Anti-muon-neutrino Induced Pi0 Production Cross Sections at 2 - $10 \mathrm{GeV}$, Doctoral Thesis, CBPF (2012).

[69] B. P. Ziemer, A measurement of the Two Track Charged-Current Quasi-Elastic Cross Section with the MINER $\nu$ A Detector; Doctoral Thesis; University of California, Irvine (2012).

[70] J. Chvojka, Anti-Neutrino Charged Current Quasi-Elastic Scattering in MINER $\nu$ A, Doctoral Thesis, University of Rochester (2012).

[71] J. Allison et al., IEEE Transactions on Nuclear Science 53 No. 1, 270-278 (2006).

[72] C. Alt et al., Inclusive Production of Charged Pions in $\mathrm{p}+\mathrm{C}$ Collisions at $158 \mathrm{GeV} / \mathrm{c}$ Beam Momentum, Eur. Phys. J. C 49, 897-917 (2007). 
[73] C. Anticic et al., Inclusive Production of Charged Kaons in p + p Collisions at $158 \mathrm{GeV} / \mathrm{c}$ Beam Momentum and a New Evaluation of the Energy Dependence of Kaon Production up to Collider Energies, Eur. Phys. J. C 68, 1-73 (2010).

[74] D.S. Barton et al. Experimental Study of the A Dependence of Inclusive Hadron Fragmentation, Phys. Rev. D 27 2580, (1983).

[75] N. Tagg et. al.; Arachne - A web-based event viewer for MINERvA; Nucl.Inst.Meth. 676 44-49 (2012).

[76] Minerva Collaboration: L. Fields et. al., Measurement of Muon Antineutrino Quasi-Elastic Scattering on a Hydrocarbon Target at $E_{n} u \sim 3.5 \mathrm{GeV}$, Phys. Rev. Lett. 111, 022501 (2013).

[77] Minerva Collaboration: G. A. Fiorentini et. al., Measurement of Muon Neutrino Quasielastic Scattering on a Hydrocarbon Target at $E_{\nu} \sim 3.5 \mathrm{GeV}$, Phys. Rev. Lett. 111, 022502 (2013).

[78] G. D' Agostini, A multidimensional unfolding method based on Baye's theorem, Nucl. Instrum. Methods in Phys. Res. Sect. A 362 487-49 (1995). 